\title{
Monitoring Breathing Using a Doppler Radar
}

\author{
by
}

Philip Tworzydlo

A thesis submitted to the Faculty of Graduate and Postdoctoral Affairs in partial fulfillment of the requirements for the degree of

Master of Applied Science

in

Biomedical Engineering

Carleton University

Ottawa, Ontario

(C) 2016, Philip Tworzydlo 


\begin{abstract}
Correctional institutions are looking to adopt a non-obtrusive method for monitoring the vital signs of inmates in Canada. Doppler radar has been previously investigated as a potential method of vital signs detection and monitoring. This thesis presents an algorithm which uses the baseband output signal received by a Doppler radar to estimate the breathing rate from the detected motion associated with breathing. A reliability measure is provided with breathing rate estimates through the analysis of various signal quality indices. Results demonstrate that the algorithm is able to estimate the subject's breathing rate (with high reliability) when the subject is motionless and breathing normally (mean error of 6 breaths per minute). The algorithm cannot accurately estimate the breathing rate when the subject is moving or holding their breath (mean error of 21 breaths per minute). During breath holds, it was found that the algorithm can likely estimate the subject's heart rate.
\end{abstract}




\section{Acknowledgements}

First and foremost, I would like to thank my supervisor, Dr. Adrian D.C. Chan, for his guidance, encouragement, and patience throughout this whole experience. His guidance and knowledge helped me complete my research, and write a clear and concise thesis.

Besides my supervisor, I would like to thank André Gagnon, and others from K\&G Spectrum, for letting me use their equipment to conduct my research, and for their support in answering my questions.

I would also like to thank all my friends (especially Mike, Matt, Seamus, and Mark) for their encouragement, for sharing some laughs with me, and reminding me to take the time to relax and enjoy myself from time to time.

Lastly, I would like to thank my family, especially my parents for their unwavering support and constant encouragement throughout the writing of this thesis, and my life in general. 


\section{Table of Contents}

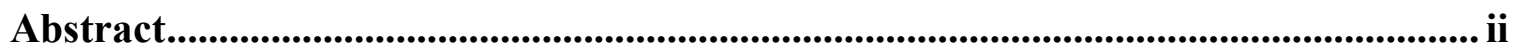

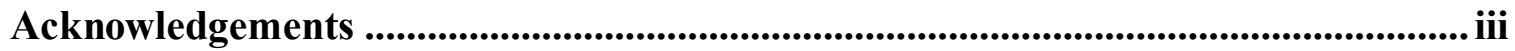

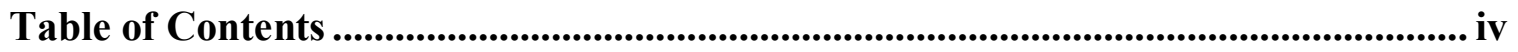

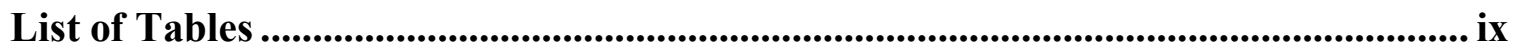

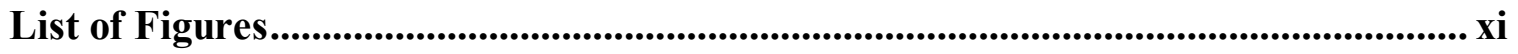

1 Chapter: Introduction ........................................................................................... 1

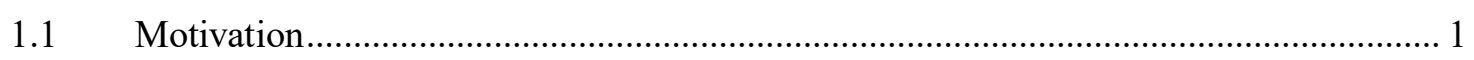

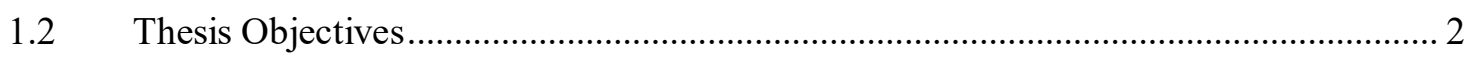

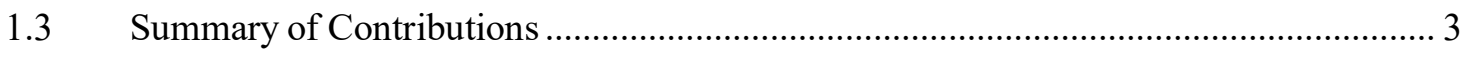

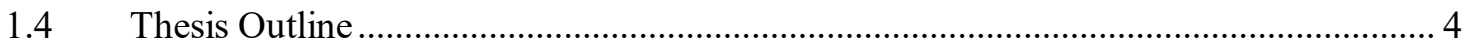

2 Chapter: Background .....................................................................................6

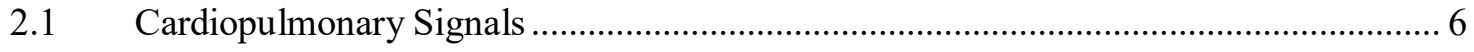

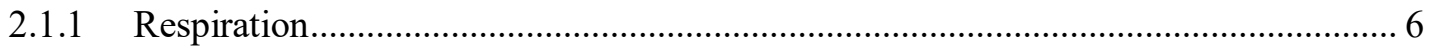

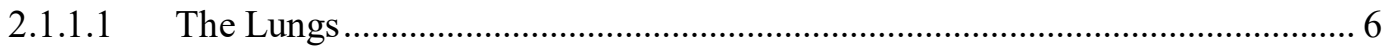

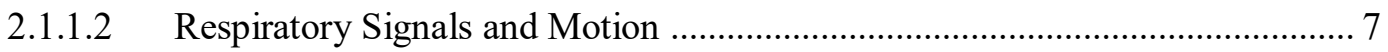

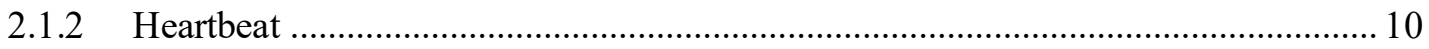

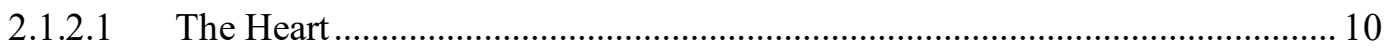

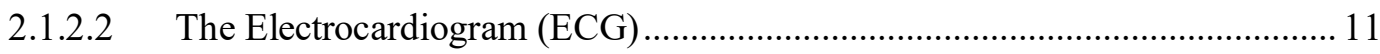

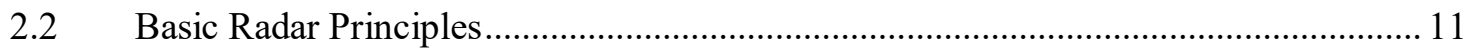

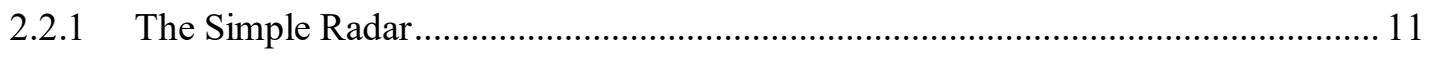

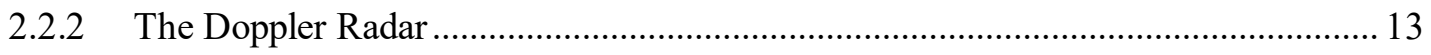

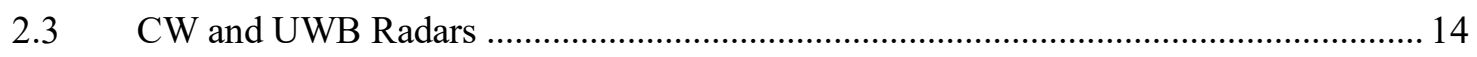

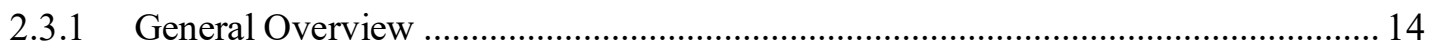

2.3.2 Safety Concerns Related to Electromagnetic Radiation ......................................... 16 


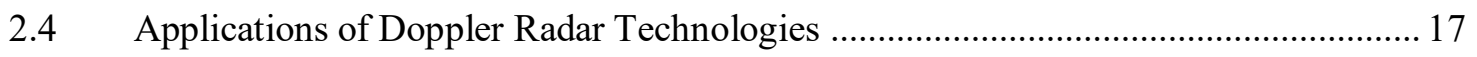

2.4.1 Vital Signs Monitoring with Doppler Radar ........................................................ 18

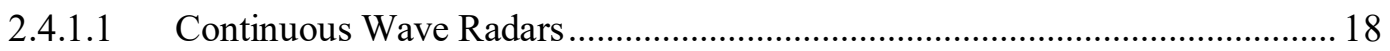

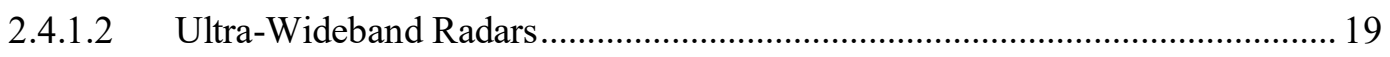

2.4.2 Advances in Technology and Signal Processing ................................................... 21

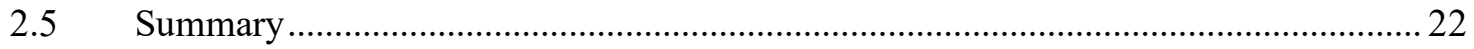

3 Chapter: Experimental Setup and Equipment ..................................................... 24

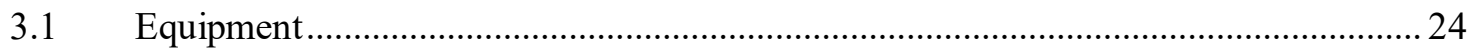

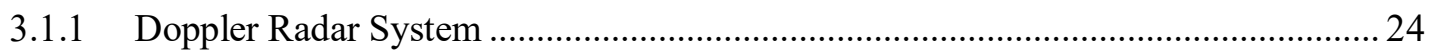

3.1.2 Respiratory Inductance Plethysmography (RIP) Band …................................... 27

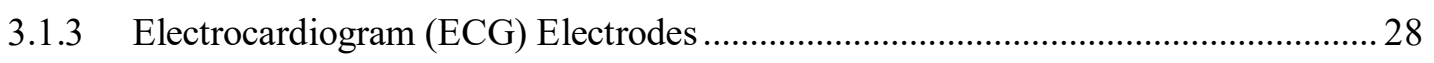

3.1.4 Bioinstrumentation Amplifier and Data Acquisition Unit (DAQ) .......................... 28

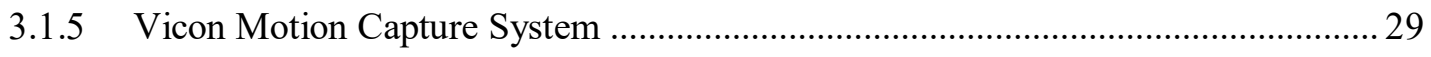

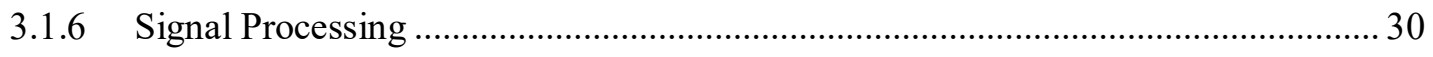

4 Chapter: In-Phase Quadrature (I/Q) Testing ................................................. 31

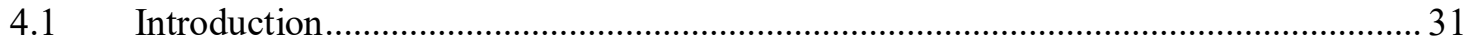

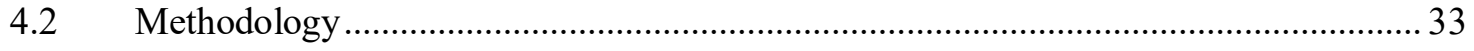

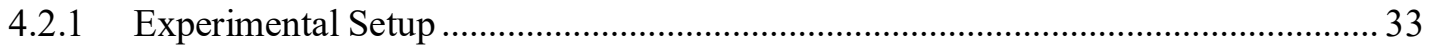

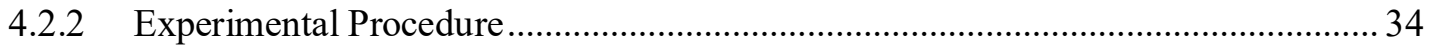

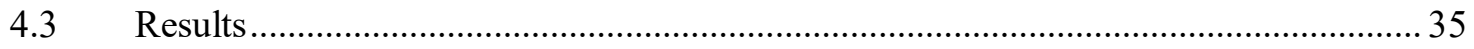

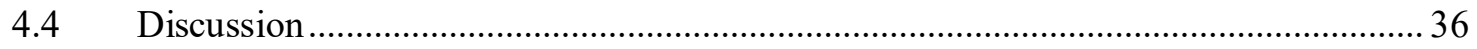

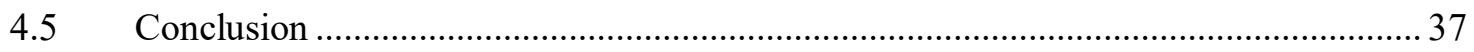

5 Chapter: Breathing Estimation Algorithm .............................................................. 38

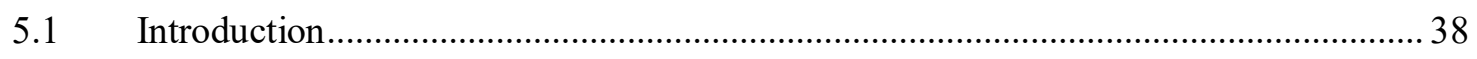

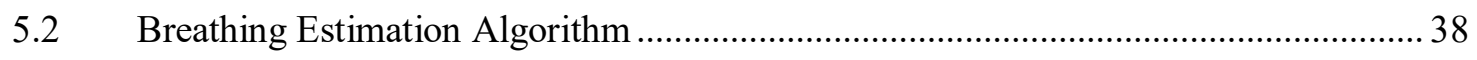




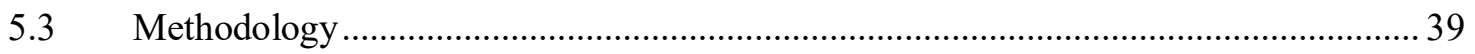

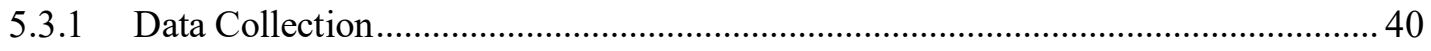



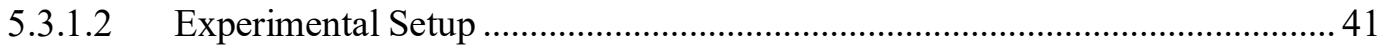

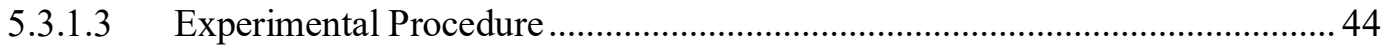

5.3.2 Estimation of Breathing and Heart Rate ................................................................... 45

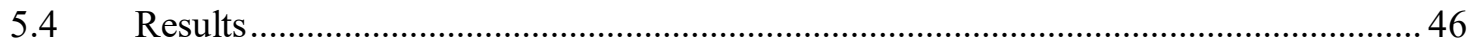

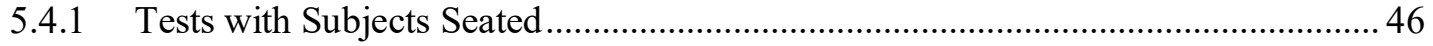

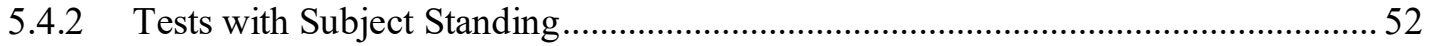

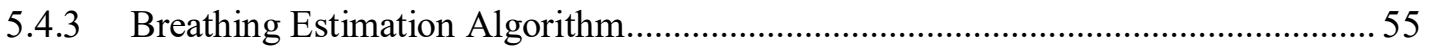

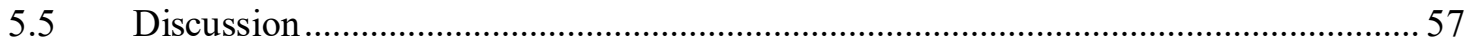

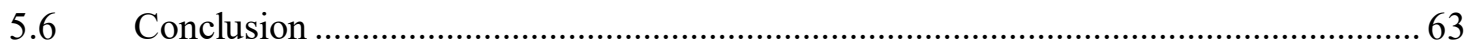

6 Chapter: Breathing Estimate Reliability Measure ............................................. 65

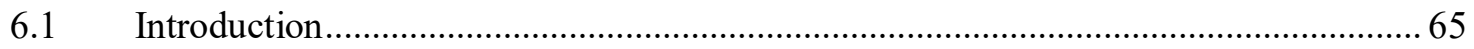

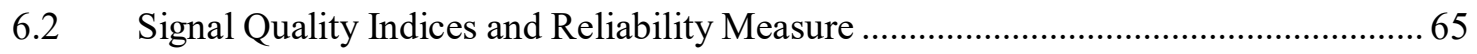

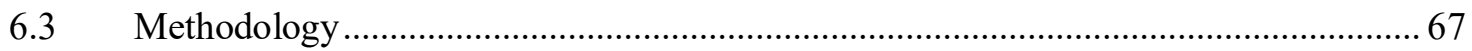

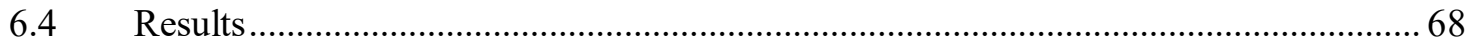

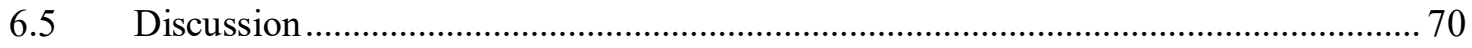

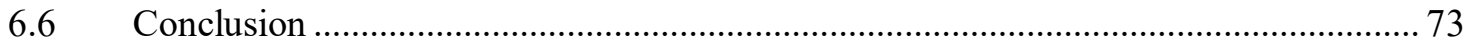

7 Chapter: Analysis of Breathing Motion..................................................................... 75

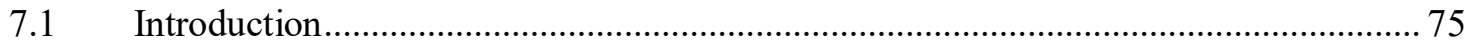

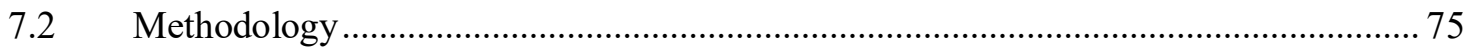

7.2.1 Effect of Orientation and Determination of Signal Source …................................. 75

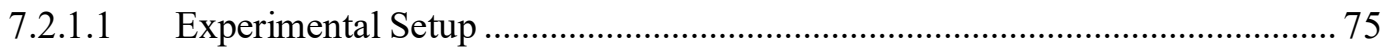

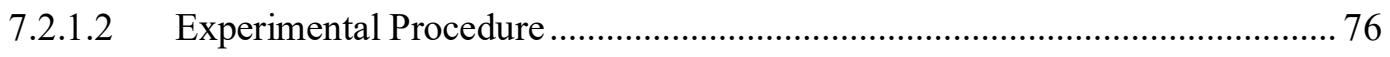

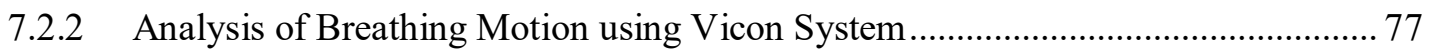




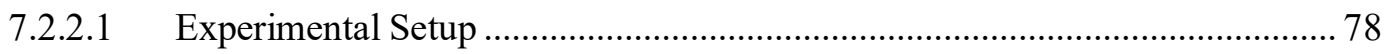

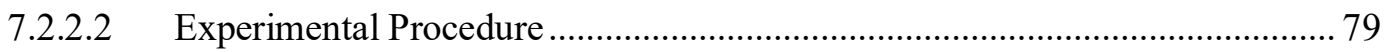

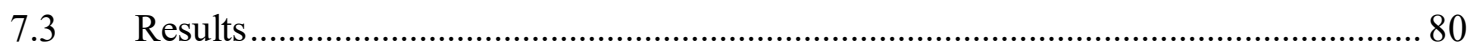

7.3.1 Effect of Orientation and Determination of Signal Source ….................................. 80

7.3.2 Analysis of Breathing Motion using Vicon Analysis ............................................. 88

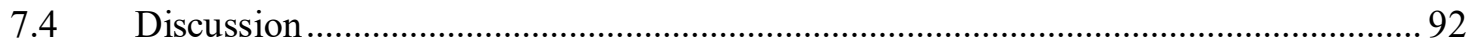

7.4.1 Effect of Orientation and Determination of Signal Source …................................... 92

7.4.2 Analysis of Breathing Motion using Vicon System ................................................ 93

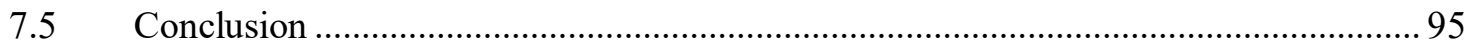

8 Chapter: Breathing Simulator .................................................................... 97

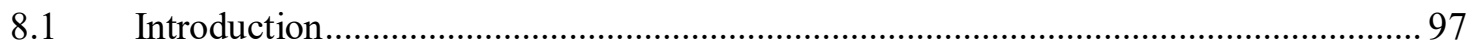

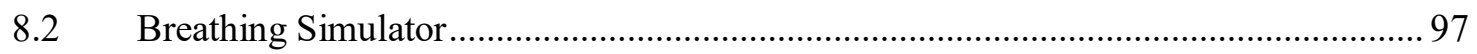

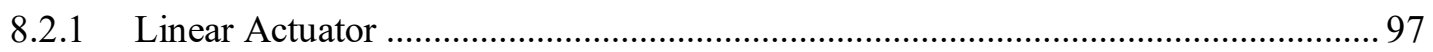

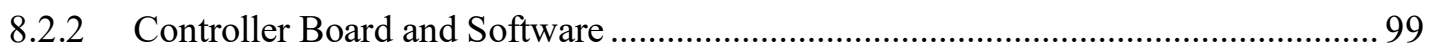

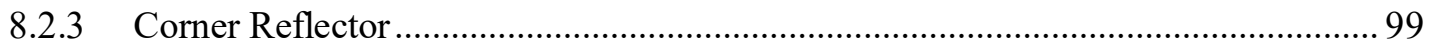

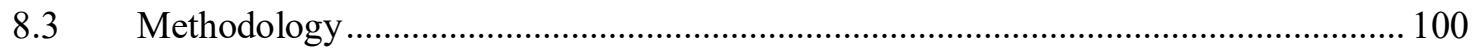

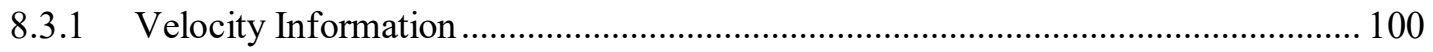

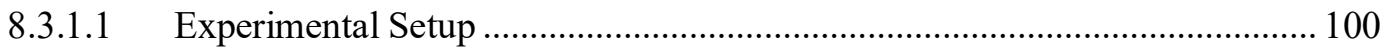

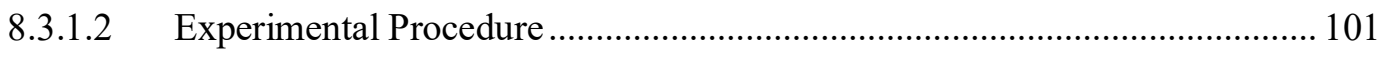

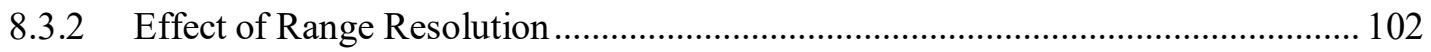

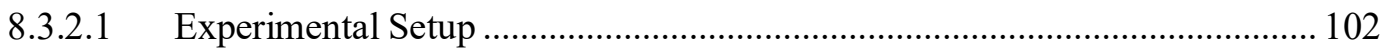

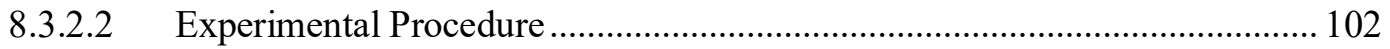

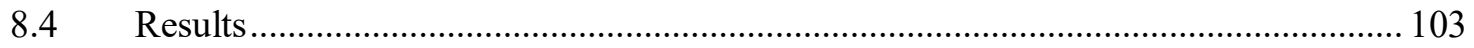

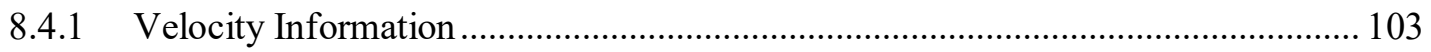

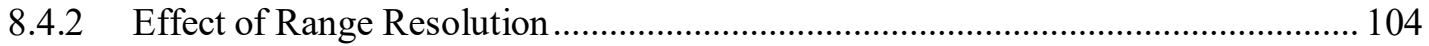

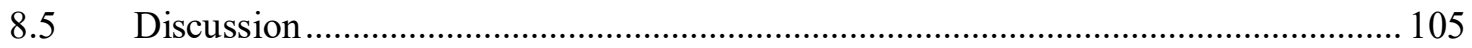




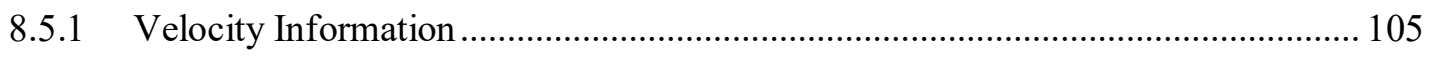

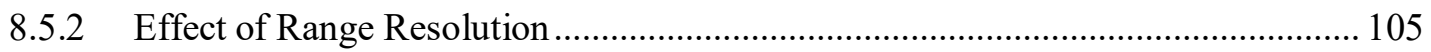

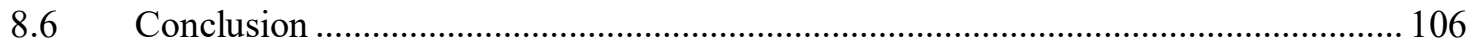

9 Chapter: Conclusions and Recommendations for Future Work ...................... 107

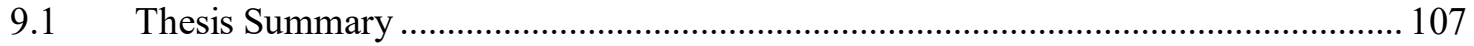

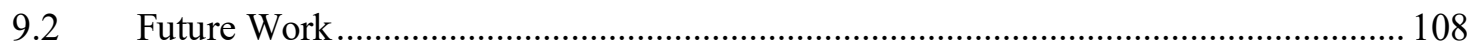






\section{List of Tables}

Table 2.1 Electromagnetic and anatomical properties of tissue layers in the thorax.

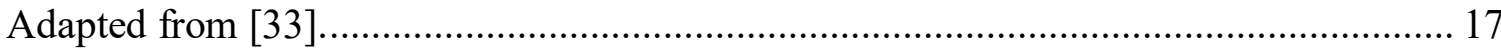

Table 3.1 Doppler radar units used in this research................................................... 25

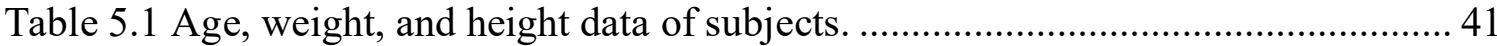

Table 5.2 Radar unit and orientation setup for testing with each subject. ...................... 42

Table 5.3 Primary peak locations of the radar output signal obtained from the magnitude spectrums and the calculated breathing rate of the RIP band signals corresponding to the analysis windows from Figure 5.4. 51

Table 5.4 Primary peak locations of the radar output signal obtained from the magnitude spectrums and the calculated breathing rate of the RIP band signals corresponding to the analysis windows from Figure 5.7. 55

Table 5.5 Mean and standard deviation of breathing estimate error in each breathing segment for all sitting tests from each subject...

Table 5.6 Mean and standard deviation of breathing estimate error in each breathing segment for all standing tests from each subject.

Table 5.7 Mean and standard deviation of heart rate estimate error from breath hold segment for all sitting and standing tests from each subject, and average heart rate over the breath hold for each subject. 62

Table 7.1 Physical traits of each subject that partook in the Vicon testing. ..................... 78

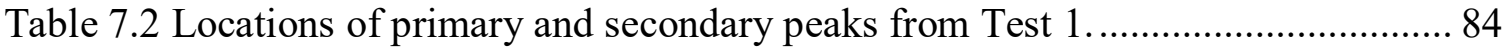

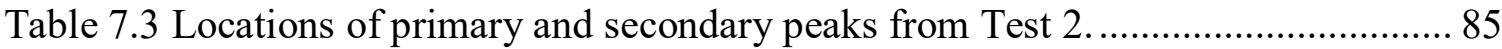

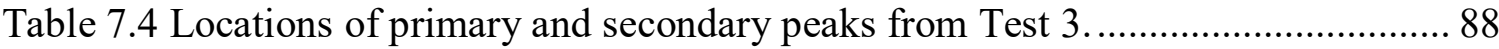


Table 8.1 Specifications for the Firgelli linear actuator.

Table 8.2 Signal and noise power values used to calculate the SNR of the radar output signals from Tests 3 and 4. 


\section{List of Figures}

Figure 2.1 The lungs with key parts indicated [9] ................................................... 7

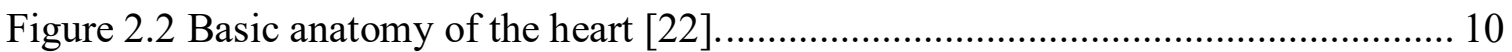

Figure 2.3 Electrophysiology of the heart and the resultant generation of ECG signal

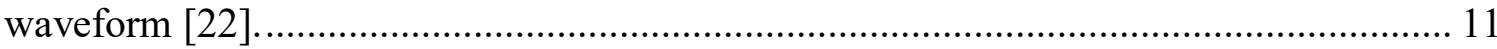

Figure 2.4 Radar block diagram. Adapted from [23]............................................... 12

Figure 2.5 Block diagram of the Pseudorandom Spread Spectrum CW Doppler radar used

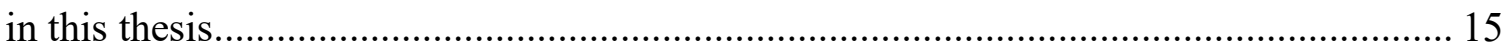

Figure 3.1 The SR12003 radar unit in its vertical orientation (left) and horizontal

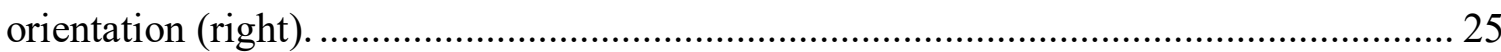

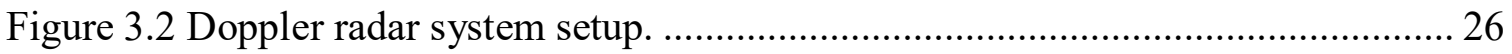

Figure 3.3 KGScope radar configuration setup screen. ............................................... 27

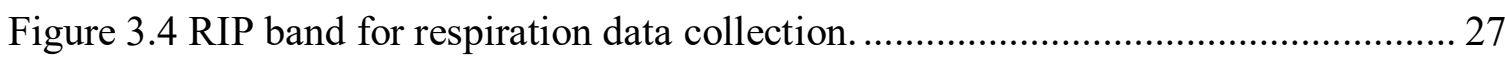

Figure $3.5 \mathrm{Ag} / \mathrm{AgCl}$ electrodes for $\mathrm{ECG}$ data collection. ................................................ 28

Figure 3.6 Floorplan of the Motion Capture Lab at Carleton University (modified from

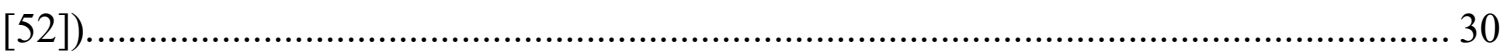

Figure $3.714 \mathrm{~mm}$ reflective marker used in Vicon motion capture. ............................... 30

Figure 4.1 Picture of measurement environment facing away from the SR12005 radar unit. 34

Figure 4.2 Subject position and experimental setup of radar unit. ................................... 34

Figure 4.3 Scatterplot of Radar's Zone 2 signal power versus its position relative to

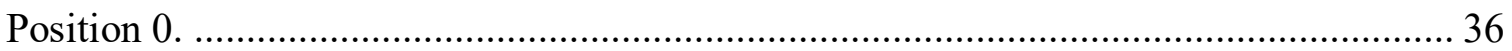

Figure 5.1 View of Vicon room and experimental setup.............................................. 42 
Figure 5.2 ECG and RIP band connections to subject.

Figure 5.3 Radar output signal from the SR12003 radar unit (top), RIP band signal (middle) and ECG signal (bottom) from subject 3's third sitting test with all breathing segments (RgBr, PA, RcBr, BrH, DBr) labelled. 48

Figure 5.4 Thirty second windows of the regular breathing, recovery breathing, deep breathing, physical activity, and breath hold segments from Subject 3's third sitting test.

Figure 5.5 Magnitude spectrum plots for the regular breathing, recovery breathing, deep breathing, physical activity, and breath hold segments of the radar (top row) and RIP band (bottom row) signals corresponding to the analysis windows from Figure 5.4 ...... 50 Figure 5.6 Radar output signal from the SR12003 radar unit (top), RIP band signal (middle), and ECG signal (bottom) from subject 3's third standing test.

Figure 5.7 Thirty second windows of the regular breathing, recovery breathing, deep breathing, physical activity, and breath hold segments from Subject 3's third standing test.

Figure 5.8 Magnitude spectrum plots for the regular breathing, recovery breathing, deep breathing, physical activity, and breath hold segments of the radar (top row) and RIP band (bottom row) signals corresponding to the analysis windows from Figure 5.7 ...... 54 Figure 5.9 Box plots of the breathing estimate error for all five breathing segments from the sitting tests of each subject (subject 1 - blue, subject 2 - red, subject 3 - green, subject 4 - black, subject 5 - magenta). 56 
Figure 5.10 Box plots of the breathing estimate error for all five breathing segments from the standing tests of each subject (subject 1 - blue, subject 2 - red, subject 3 - green, subject 4 - black, subject 5 - magenta). 56

Figure 5.11 Plot of regular breathing segments from test with radar in horizontal orientation (top), test with radar in vertical orientation (middle), and from test using the SR2003 radar unit. 58

Figure 5.12 Radar and RIP band magnitude spectrums showing the presence of harmonic peaks with larger amplitudes than the peak which should be associated with the breathing rate. 60

Figure 5.13 Magnitude spectrum of Radar (top), RIP band (middle), and ECG (bottom) signal during breath hold spectrum from subject 3's third sitting test. 61 Figure 6.1 Single time window which highlights the signal and noise segments used to calculate the Signal-to-Noise SQI 67

Figure 6.2 Box plot of SNR values in each breathing segment from sitting tests. 68

Figure 6.3 Box plot of SNR values in each breathing segment from standing tests........ 68 Figure 6.4 Box plot of coherence values in each breathing segment from sitting tests.... 69 Figure 6.5 Box plot of coherence values in each breathing segment from standing tests. 69 Figure 6.6 Box plot of variance values in each breathing segment from sitting tests. .... 70 Figure 6.7 Box plot of variance values in each breathing segment from standing tests... 70 Figure 6.8 Plots of the radar output signal (top), breathing rate estimates from the algorithm and RIP band (second from top), the breathing rate estimate error (third from top), SNR values (third from bottom), coherence values (second from bottom) and variance values (b bottom) throughout subject 3's third sitting test.... 72 
Figure 7.1 RF Panel positions. Position 1 (top left), position 2 (top right), position 3 (bottom left), and position 4 (bottom right).

Figure 7.2 Marker Placement for Vicon testing. Red markers refer to reference markers, orange markers relate to the upper thorax region, green to the lower thorax, blue to the upper abdomen, and purple to the lower abdomen. Modified from [60]

Figure 7.3 Filtered radar output signal from Test 1 where the subject is breathing normally while changing their orientation. 81

Figure 7.4 Zoomed in plots of the front-facing (top), side-facing (middle), and backfacing (bottom) segments from Test 1. 82 Figure 7.5 Filtered radar output signal from Test 2 where the subject is holding their breath after changing their orientation. 82 Figure 7.6 Zoomed in plots of the front-facing (top), side-facing (middle), and backfacing (bottom) segments from Test 2. 83

Figure 7.7 Magnitude spectrums for front-facing segment (top), side-facing segment (middle), and back-facing segment (bottom) from Test 1.

Figure 7.8 Magnitude spectrums for front-facing segment (top), side-facing segment (middle), and back-facing segment (bottom) from Test 2.

Figure 7.9 Filtered radar output signal from Test 3 where the subject is changing the position of an RF panel in each segment.

Figure 7.10 Zoomed in plots from Test 3 with the RF panel in position 1 (top left), position 2 (top right), position 3 (bottom left), and position 4 (bottom right). 87

Figure 7.11 Magnitude spectrums from Test 3 with the RF panel in position 1 (top left), position 2 (top right), position 3 (bottom left), and position 4 (bottom right). 88 
Figure 7.12 Standard deviations (in $\mathrm{mm}$ ) of the reference marker for each position. 89

Figure 7.13 Standard deviations (in $\mathrm{mm}$ ) of each subject's markers when subjects were lying down.

Figure 7.14 Standard deviations (in $\mathrm{mm}$ ) of each subject's markers when subjects were seated

Figure 7.15 Standard deviations (in $\mathrm{mm}$ ) of each subject's markers when subjects were standing. 91

Figure 7.16 Mean standard deviations (in $\mathrm{mm}$ ) of markers for each position. 91

Figure 8.1 The Firgelli L16-P linear actuator. 98

Figure 8.2 Load Curves for the L16-P miniature linear actuator. 98

Figure 8.3 Firgelli LAC Configuration Utility program used to control the L16-P. 99 Figure 8.4 L16-P linear actuator with corner reflector attached. 100 Figure 8.5 Experimental setup and placement of linear actuator relative to the radar unit for Tests 1 and 2 . 101

Figure 8.6 Experimental setup and placement of linear actuator relative to the radar unit for Tests 3 and 4 with views from behind the linear actuator (left) and from the side of the radar and linear actuator (right). 102

Figure 8.7 Radar output signals from the manual cycle from Test 1 (top left), the 50 automated cycles from Test 2 (bottom left), three cycles from the automated test with two cycles highlighted in red (top right), and the approximation of the velocity-time profile of the linear actuator (bottom right). 103 
Figure 8.8 Twenty second snapshots of the Zone 5 (top), Zone 1 (middle) time domain signals from Tests 3 and 4 respectively, and the comparison between the two signal waveforms (bottom) 


\section{Chapter: Introduction}

\subsection{Motivation}

In recent years, incidents of self-harm/injury and suicide in federal penitentiaries and correctional institutions have risen. Between 2007 and 2012, the cases of self-injury in Canada nearly tripled (with the most pronounced increase seen among female prisoners, and throughout correctional institutions in the Prairies) [1], [2], [3]. When an individual is sentenced to a correctional institution/prison, the institution has a legal duty of care to take positive measures to protect all inmates (especially those who are mentally ill or particularly vulnerable) from self-harm, dying or any abuse while under their custody [4]. The most common forms of self-injury seen in Canadian correctional institutions involve prisoners slashing their forearms, mouths and body cavities, burning, banging their heads and choking themselves behind bars [3], [4]. The most common methods of suicide include overdose of psychotropic drugs, antihypertensives, and pain medication, and hanging (suspended by a ligature) [5]. Hanging accounts for between 70 to 80 percent of completed suicides [5], [6]. Asphyxia is the main cause of death in hanging [5].

These types of incidents are a problem for correctional institutions as they bring to light their failures to protect inmates in custody, and their lack of comprehensive mental health needs assessments and treatment plans. These incidents lead to tarnished reputations, and place large burdens on the institutions such as increased litigation costs, and an increased need for continuous monitoring [7]. Current monitoring methods include security rounds, counts, and patrols where guards move cell to cell monitoring the conditions of inmates, and video surveillance. Both of these methods are not without issue. There are compliance issues related to the quality and frequency of security patrols, and poor guard 
training can result in deficiencies in monitoring suicide pre-indicators, or failing to verify a living, breathing body [4]. Cameras have blind spots, may not be able to verify a living, breathing body (such as a person sleeping under a blanket), and introduce concerns related to privacy and over-reliance.

An improved method of inmate monitoring is needed. This method should be capable of monitoring an inmate's life or vital signs, should not impact the liberty and security of the inmate, and should be compliant with applicable policy and legal safeguards. This method should also be contactless since it will be used to monitor inmates who may be non-compliant (they cannot be made to wear any monitoring device and it should be kept away from them for safety reasons). This thesis examines the potential of a Doppler radar system for vital signs monitoring of inmates. The Doppler radar system is nonobtrusive and respects the rights of inmates. If an inmate is moving around the room, they are clearly alive. The Doppler radar system can potentially help to determine what activity the inmate is doing inside the room, but this is outside the scope of this thesis. Additionally, it will be difficult to estimate breathing rate when an inmate is moving, but given that there is a verification of liveness, a breathing estimate is not needed. Accurate breathing estimation during motion is also outside the scope of this thesis.

\subsection{Thesis Objectives}

The overall objective of this thesis is to investigate Doppler radar technology as a contactless method of vital signs monitoring for use in correctional institutions; in particular breathing detection. This thesis presents the following specific objectives:

1. Implement an algorithm which estimates a subject's breathing rate from the Doppler radar output signal. 
2. Develop and evaluate a signal quality index that can be used to quantify the reliability of the breathing rate estimate.

3. Investigate and provide an objective analysis of breathing motion, and relate the breathing motion to the Doppler radar output signal.

\subsection{Summary of Contributions}

This thesis presents the following major contributions:

\section{Implementation and evaluation of a breathing estimation algorithm}

Doppler radar technology has been proposed as a method of vital signs monitoring for inmates in correctional institutions, and there is evidence of its ability to detect breathing motion. This thesis presents an algorithm which uses the Doppler radar output signal associated with a subject's breathing motion to estimate their breathing rate. To determine the accuracy of the algorithm, the breathing estimate from the radar output signal is compared to the signal from a RIP band. This algorithm is meant to serve as a baseline level of performance that future research can use to compare against. While this thesis focuses on the application of the radar technology in correctional institutions, the breathing estimation algorithm may be applicable in other areas requiring respiratory monitoring such as sleep apnea studies or infant care.

\section{Development and evaluation of a signal quality index as a reliability measure of} the breathing rate estimate

Various SQIs (signal-to-noise ratio, magnitude squared coherence, and variance) are investigated to provide a reliability measure for each estimate. This reliability measure can be applied to an alarm system, sounding the alarm when the reliability is 
high and the breathing estimate is outside a specified threshold, and preventing false alarms by ignoring estimates when reliability is low.

\section{Analysis of breathing motion using Vicon motion tracking/capture system}

Despite there being evidence that Doppler radar technology is capable of detecting breathing motion, very little is known about how different areas of the upper body move during breathing, and how this affects the radar output signal. To investigate the source of the radar breathing signal, an objective analysis of breathing motion was conducted using the Vicon motion tracking system. The effect of position was investigated as well as the effect of the subject's orientation relative to the radar. Results showed that position determines how much different areas of the upper body move during breathing, and that all recordings should be taken with the subject facing the radar unit.

A portion of this research was disseminated in the following paper:

Tworzydlo P, Chan ADC, "Spectral Analysis of Respiratory and Cardiac Signals Using Doppler Radar", World Congress on Medical Physics and Biomedical Engineering, Toronto, Canada, IFMBE Proceedings vol. 51, pp. 1034-1038, 2015.

\subsection{Thesis Outline}

The remainder of this thesis is structured as follows. Chapter 2 provides background information on the basic principles of radar technology, as well as biosignal data from the cardiovascular and respiratory systems. Additionally, Chapter 2 contains a literature review of the analysis of breathing motion and breathing patterns, and the application of radar technology in vital signs monitoring, specifically, contactless breathing detection and heartbeat monitoring. Chapter 3 outlines the methodology used throughout the course 
of this thesis; providing specific information on the equipment and software used to perform the research. Chapter 4 discusses the I/Q effect of radar systems and investigates the need for I/Q demodulation in radar units for vital signs monitoring. Chapter 5 presents the breathing estimation algorithm. Chapter 6 discusses the validation of the algorithm's breathing estimates using various SQI. Chapter 7 provides an analysis of breathing motion and relates it to the radar output signal. Chapter 8 presents preliminary work with a repeatable motion system using a linear actuator. Finally, Chapter 9 summarizes the contents of this thesis and proposes future work to further the research presented herein. 


\section{Chapter: Background}

This thesis investigates the use of a Doppler radar system for vital/life signs monitoring of inmates in correctional institutions. The aim of this thesis is to determine if Doppler radar can monitor breathing motion, and whether it can detect the small movements of the chest associated with the heartbeat, for the purposes of life signs monitoring. This chapter begins with a discussion on the lungs, heart, and data collection of respiratory and cardiac signals. Later sections provide an overview of radar principles, and discuss existing literature on the use of radar systems for detection and monitoring of life signs, and breathing motion. This chapter aims to provide a context which will help the reader understand the contributions presented in this thesis and the reasoning behind the approach that was used.

\subsection{Cardiopulmonary Signals}

\subsubsection{Respiration}

\subsubsection{The Lungs}

The lungs (Figure 2.1) are the principle organs of the respiratory system where the net diffusion of oxygen from air to blood, and the net diffusion of carbon dioxide from blood to air occur. The lungs are separated into two zones: 1) the conducting zone, through which fresh air is channeled for ventilation, and 2) the respiratory zone, the region where gas exchange with the blood occurs [8]. The conducting zone consists of the trachea, or windpipe, which branches to form smaller bronchi and even smaller bronchioles [8]. At the terminal bronchioles, air is channeled into the respiratory zone where gas exchange occurs across the walls of alveoli (small sac-like dilations) [8]. The upper body can be separated into a lower abdominopelvic cavity and an upper thoracic cavity (or thorax) 
and during inspiration (inhalation) or expiration (exhalation), the volume of both the lungs and thorax change during breathing, increasing during inspiration and decreasing during expiration. Changes in thoracic volume are caused by the contraction and relaxation of skeletal muscles, the chief of these being the diaphragm, a dome-shaped sheet of striated muscle which curves upward into the thoracic cavity while at rest [8].

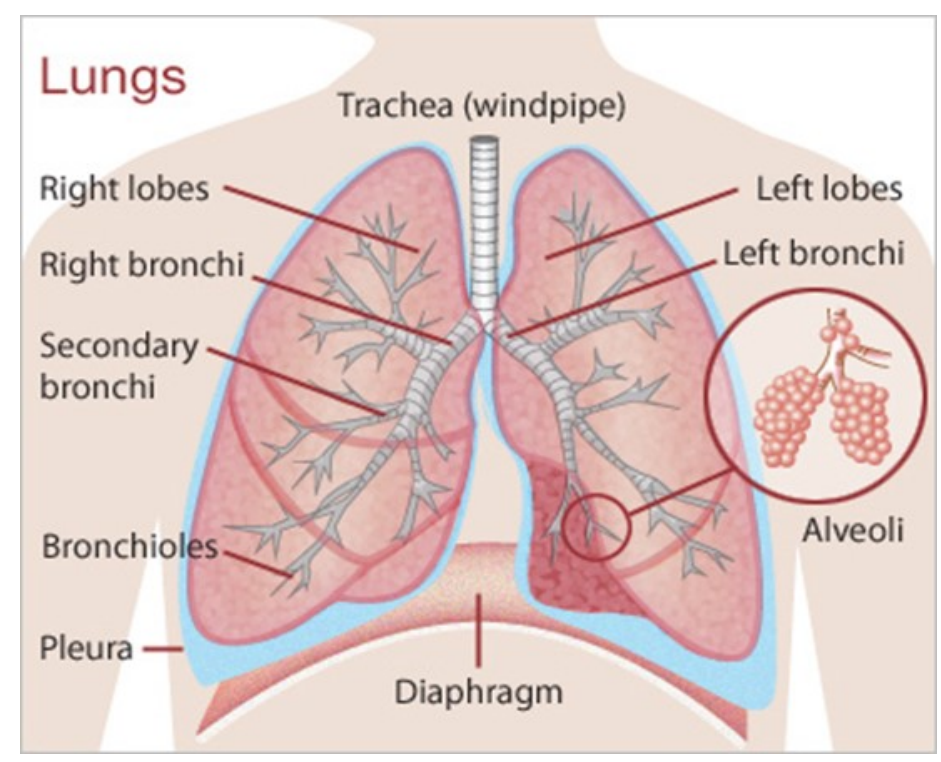

Figure 2.1 The lungs with key parts indicated [9].

\subsubsection{Respiratory Signals and Motion}

Pulmonary function is often tested using a spirometer, a device which measures the amount of air inhaled or exhaled during breathing [8], [10]. Respiration can also be measured using a respiratory inductance plethysmograph (RIP) band around a person's thorax, which is used in this thesis. Research has been done to analyse the motion of the upper body associated with breathing [11]-[21].

An optical reflectance system combining image processing and parallel computing were investigated as alternate methods for breathing analysis in [11] and [12]. A computer vision system (ELITE) paired with a television-image processor was proposed in [11] to 
provide a kinematic analysis of chest wall and lung volume during breathing. Subjects had passive reflective markers placed at specific body landmarks, and were observed using four specially designed television cameras which recognized the markers and computed their two-dimensional coordinates [11]. Post-signal processing and analysis confirmed the method's ability to monitor the motion of the chest wall, and estimate the change in volume of the chest. Researchers in [12] assessed the previous optical reflectance system by comparing the system's estimates of the changes in chest wall and lung volume to values estimated using spirometry. There was good correlation between the two estimates which showed the system's viability for chest wall and lung volume analysis [12].

A similar system was presented in [13] which used image and signal processing techniques to extract chest and abdominal movement information. The only difference with this system was that it extracted the movement information from a sequence of video images recorded using a single camera. Subjects were recorded (different shirt colours and patterns were worn to determine if there was any effect on the accuracy) producing sequential images which were stacked, and a specially designed algorithm was developed to perform image subtraction between the current and previous images [13]. Movement information was extracted and a plot of the breathing signal was generated along with an estimate of breathing rate. When compared to more conventional methods of measuring pulmonary function, the proposed method generated accurate breathing rate results, confirming its effectiveness. No information was provided regarding the range of motion of the chest or abdomen during breathing. 
In recent years, new instruments/devices have been proposed for measuring respiratory/breathing movement. Researchers in Sweden developed a Respiratory Movement Measuring Instrument (RMMI) [14], while researchers in Japan developed a Breathing Movement Measuring Device (BMMD) [15], [16]. These handheld devices make contact with subjects and have reported success and reliability in measuring breathing movement. The RMMI consists of six laser distance sensors held on two rods secured to a pole [14]. The rods and four of the sensors are moveable to account for differences in chest width and length. The BMMD comprises a syringe with a millimeter scale, angle gauge, a tension spring, a metal rod, a coupling part with a mark, and a discoid contact part with a ball joint [16]. Breathing movement is estimated from the position of the mark which moves on the scale through the discoid contact. Measurements were taken at specific observation points which included the third rib, and eighth rib. Different observation points were used in this thesis.

Investigations into how different physical factors including gender, posture, and age affect breathing movement have been conducted using previously mentioned methods [14]-[17]. Results have shown that breathing movements do not change significantly with increasing age, and that men and women have similar breathing movements (though men experience greater abdominal movements during deep breathing) [17], [18], [19].

Additionally, the contributions of the pulmonary and abdominal rib-cage, and abdomen, to the compartmental chest wall volume, change between standing, supine, and prone positions [20], [21]. The volume of the abdomen increases more when the subject is in a supine position compared to a prone position. 


\subsubsection{Heartbeat}

\subsubsection{The Heart}

The heart (Figure 2.2) is the primary organ of the circulatory system responsible for pumping blood around the body.

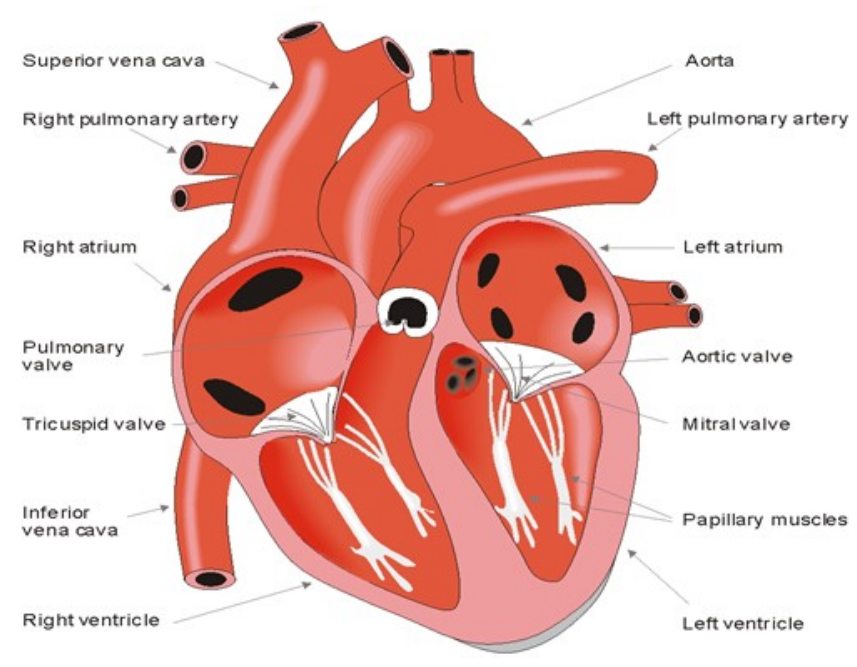

Figure 2.2 Basic anatomy of the heart [22].

The human heart has four chambers: the left and right atriums which receive blood from veins, and the left and right ventricles which pump blood into the arteries [8]. The right ventricle pumps blood into the lungs for pulmonary circulation, while the left ventricle pumps blood to tissues everywhere else around the body [8]. The heart also has two pairs of one-way valves: 1) the atrioventricular (tricuspid and mitral) valves, which separate the atria from the ventricles and only allow blood to move from the atria to the ventricles, and 2) the semilunar valves (aortic and pulmonary), which prevent blood from backing up into the ventricles from the arteries [8]. 


\subsubsection{The Electrocardiogram (ECG)}

The electrocardiogram is the electrical signal associated with the activation (depolarization and repolarization) of various locations across the heart [10]. Figure 2.3 shows how the waveforms of action potentials in the different specialized cardiac tissue combine to form the ECG [22]. ECGs are recorded by attaching a minimum of two electrodes on either side of the heart (one on the left side of the body and one on the right side of the body) [10].

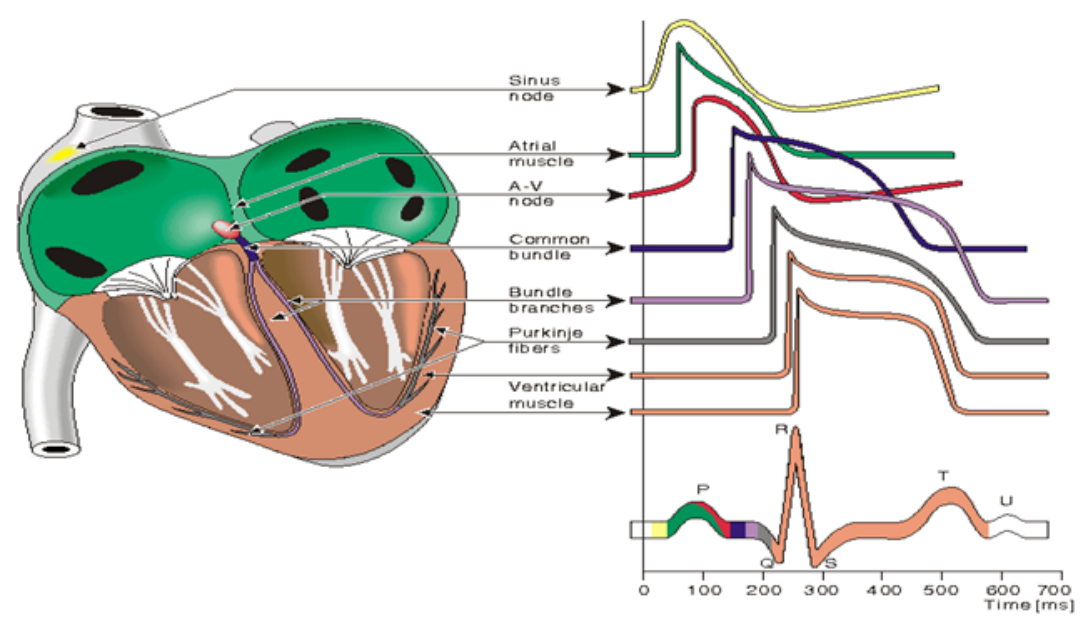

Figure 2.3 Electrophysiology of the heart and the resultant generation of ECG signal waveform [22]. The main components of an ECG are the P wave, QRS complex, and T wave [10]. Each of these components is associated with a specific electrical event in the heart. The $\mathrm{P}$ wave is produced by the depolarization of the atria, causing them to contract [10]. The QRS complex is produced when action potentials arrive at the ventricles, causing them to depolarize and the $\mathrm{T}$ wave is produced when the ventricles become repolarized [10].

\subsection{Basic Radar Principles}

\subsubsection{The Simple Radar}

Radar is an acronym for Radio Detection and Ranging. The principles of a primitive pulsed radar system (shown in Figure 2.4) are transmission, propagation, and reflection. 


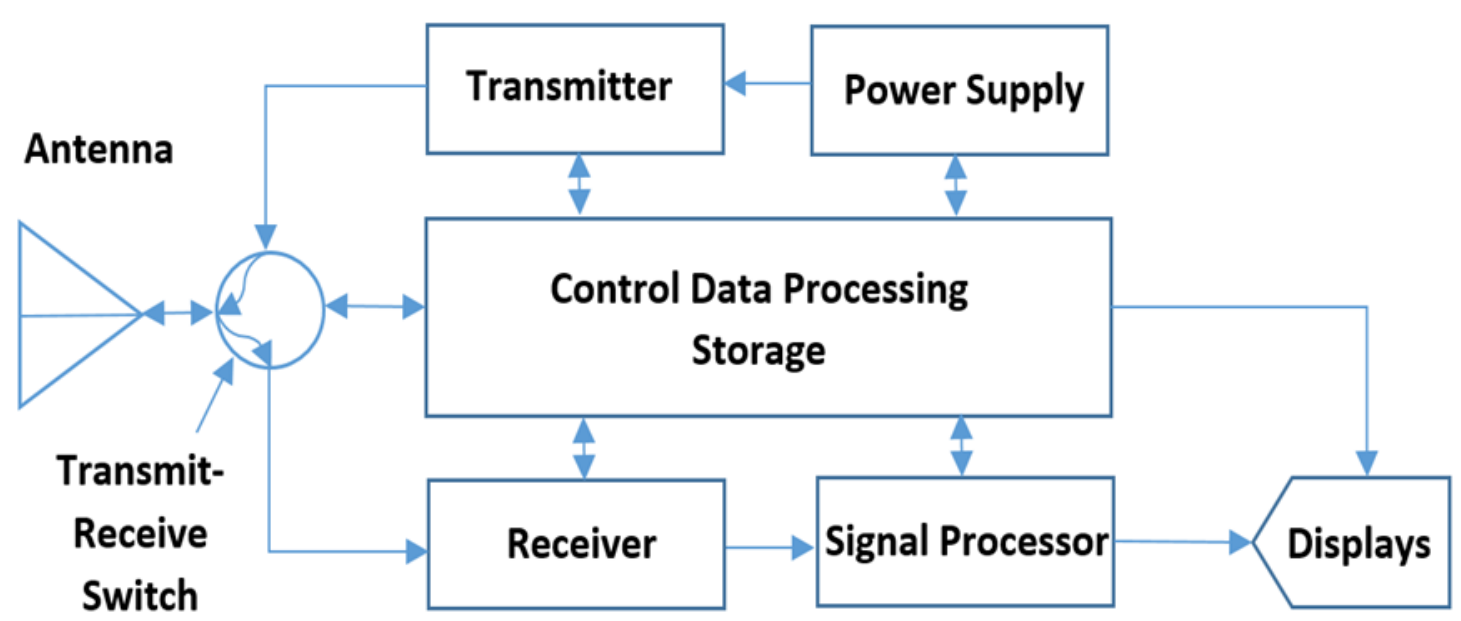

Figure 2.4 Radar block diagram. Adapted from [23].

The transmitter generates a pulse of electromagnetic energy, which oscillates at a predetermined frequency and duration. The pulse is then routed through the transmitreceive switch to the antenna which then radiates the pulse out into free space. The pulse propagates at the speed of light, scattering from objects it encounters along the way [23]. Parts of the scattered signal that return are collected by the antenna and routed through the transmit-receive switch to the receiver. The presence of the received signal can be discerned by the radar because the frequency and duration of the signal are the same as for the transmitted pulse [23]. Signal processing of the received signal involves enhancing the received signal, and removing or attenuating interfering signals [23]. The processed receive signal is presented to an operator on displays.

Estimating the distance from the radar to an object is a primary goal of radar. This distance can be calculated using basic physics. The elapsed time from when a pulse is transmitted to when the received signal is detected is measured. The distance to an object can be calculated, given that distance is equal to half the product of the elapsed time and speed (speed of light). 
Time delay can also be measured using range bins, or range zones [23]. A range bin is equal to approximately half the product of the pulse duration and the speed of light [23]. A received signal will arrive in a specific range bin based on its associated time delay. Radio waves occupy a section of the electromagnetic spectrum ranging from a few kilohertz to a few million megahertz, and the radar spectrum is subdivided into different subranges [23], [24]. The higher the transmitted frequency of the pulsed radar system, the better the range resolution; but higher frequency signals also experience greater atmospheric attenuation [25].

\subsubsection{The Doppler Radar}

Doppler radars make use of the Doppler effect (Doppler frequency shift) to measure the radial velocity of a moving object. The Doppler effect is the perceived change in frequency or pitch of an electromagnetic wave such as sound due to the relative motion between the source and the receiver [24].

When an object is moving towards a radar with a certain velocity (time rate of change of range), its radial velocity equates to the velocity component in the direction of the radar's line of sight (LOS) [23], [26]. The movement of the wave can be broken down into two segments, each producing a Doppler shift. As the wave travels from the transmitter to the object, the object will produce a Doppler frequency shift relative to its radial velocity, since the object will intercept more waves as it moves towards the radar [23], [26]. The second Doppler shift occurs as the wave travels from the object back to the receiver, since each wave that is reradiated from the moving object is reradiated at a point closer to the radar than the previous one [23], [26]. 
Doppler radar systems typically use Continuous Wave (CW) radar devices which transmit continuous and unmodulated waves with a constant frequency and amplitude (can be considered as pure sine waves) [24]. The echoes or received signals from CW radars will have the same frequency (stationary targets) or frequencies shifted by a Doppler frequency (moving targets). Because of the continuous nature of $\mathrm{CW}$ emission, range information can only be extracted by modulating the transmit wave. The most commonly used technique is Linear Frequency Modulation (LMF) which implements timing marks in the transmit and receive waves [27]. By comparing the timing marks in the transmit and receive waves, Frequency Modulated Continuous Wave (FMCW) radars can measure target range.

\subsection{CW and UWB Radars}

\subsubsection{General Overview}

With the advancement of microwave sensor technologies, a great deal of work in the research community has focused on using CW radars and Ultra-Wideband (UWB) radars for vital signs detection [28]-[51]. UWB radars transmit short pulses, with pulse duration on the order of nanoseconds, while CW radars transmit continuous waves [28]. Despite these differences, both types of radars can detect the movement of an object by measuring the Doppler shift from the received signal. CW radars without LMF capabilities, are considered more simple systems but, UWB radars have, by default, better range resolution [28]. In applications or clinical environments where multiple people or objects are moving in the same field of view as the target under observation, the range resolution of UWB radars means that measurements of the target's range and motion will not be degraded so long as the target can be discriminated from the other people/objects. 
Spread spectrum systems have a transmitted baseband signal that is modulated with a wideband encoding signal, spreading the baseband signal over a larger bandwidth; much wider than the minimum bandwidth required to transmit the information being sent [29]. UWB radars operate as spread spectrum systems; UWB is simply a modulation technique which serves to spread the baseband signal over a larger bandwidth [29]. UWB systems differ from general spread spectrum systems because their fractional bandwidth is well above $25 \%$ [29].

Figure 2.5 shows a simple block diagram of the Pseudorandom Spread Spectrum CW Doppler radars (provided by K\&G Spectrum) used in this thesis.

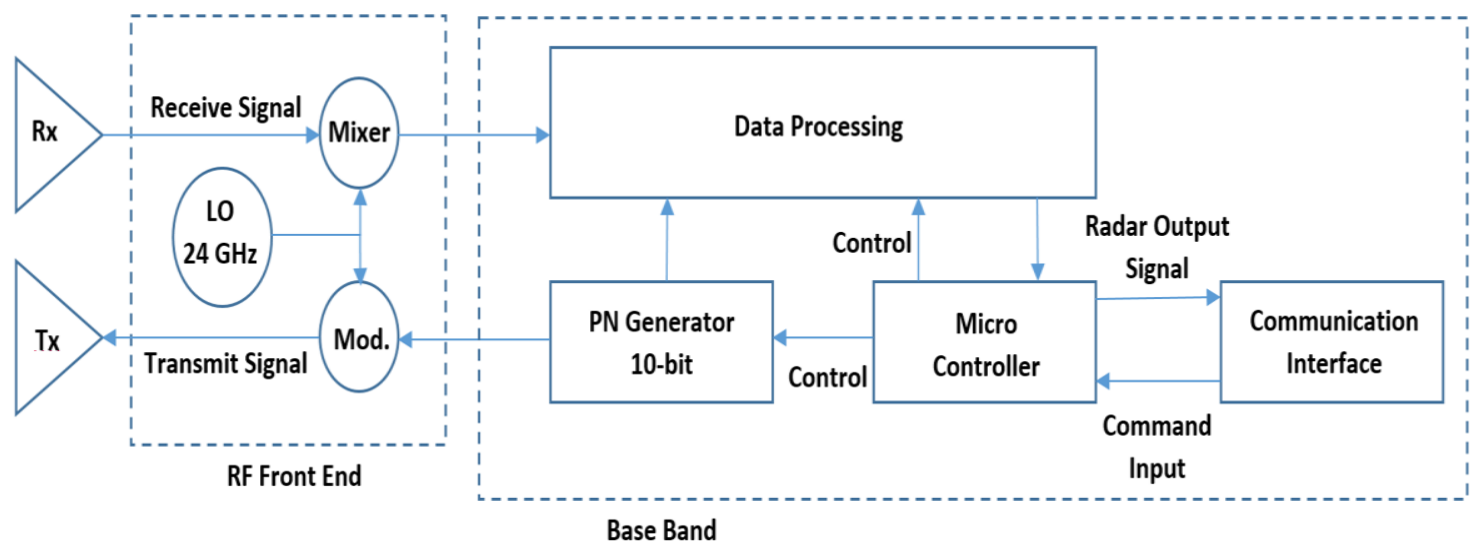

Figure 2.5 Block diagram of the Pseudorandom Spread Spectrum CW Doppler radar used in this thesis.

The radar produces the spread spectrum using a 10-bit Pseudorandom Number (PN) generator. The radar transmits a $24.125 \mathrm{GHz}$ continuous wave out into free space which reflects off a target and is then received at the antenna. The received signal is mixed with a signal from the Local Oscillator (LO) to produce an intermediate frequency, and is then processed (i.e. passed through attenuators, filters, amplifiers, and a 16-bit analog-todigital converter). The digitized data are sent to the micro controller which sends the 
demodulated baseband output signal to the communication interface. Throughout this thesis, the demodulated baseband signal is referred to as the (Doppler) radar output signal for brevity.

\subsubsection{Safety Concerns Related to Electromagnetic Radiation}

The increased use of microwave radiation in radar technologies warranted in-depth studies of the dielectric properties of various human tissues for safety analysis, and the evaluation of potential hazards to humans resulting from exposure to microwave radiation [30], [31], [32].

In [30], the then current state of knowledge of dielectric properties of tissues, based on data from the previous half century, was assessed, and gaps in knowledge were exposed in response to the need for such information in electromagnetic dosimetry. In [31], researchers measured the dielectric properties of human skin in the frequency range of 20 - $38 \mathrm{GHz}$. In [32], a novel database for biological tissues and organs was developed and comprehensive measurements systems were introduced.

Because the electromagnetic waves/pulses emitted by $\mathrm{CW}$ and UWB radars are able to probe a human body, research was conducted in [33] to understand what is measured when a UWB radar is aimed at the thorax. As part of this research, a model was studied which considered the thickness, impedance, linear attenuation, and wave speed of six superimposed living tissues to be found by the UWB pulse while travelling through the human thorax from the skin to the heart [33]. Table 2.1 lists the electromagnetic and anatomical properties of tissue layers in the thorax. 
Table 2.1 Electromagnetic and anatomical properties of tissue layers in the thorax. Adapted from [33].

\begin{tabular}{|c|c|c|c|c|}
\hline & Impedance $(\Omega)$ & Attenuation $(\mathrm{dB} / \mathrm{m})$ & Speed $(\mathrm{m} / \mathrm{s})$ & Thickness $(\mathrm{mm})$ \\
\hline Air & 376.7 & 0 & $2.998 \times 10^{8}$ & 10 \\
\hline Fat & 112.6 & 8.96 & $8.958 \times 10^{7}$ & 9.6 \\
\hline Muscle & 49.99 & 31.67 & $3.978 \times 10^{7}$ & 13.5 \\
\hline Cartilage & 58.16 & 31.93 & $4.628 \times 10^{7}$ & 11.6 \\
\hline Lung & 52.86 & 29.62 & $4.206 \times 10^{7}$ & 5.78 \\
\hline Heart & 49.17 & 38.71 & $3.912 \times 10^{7}$ & - \\
\hline
\end{tabular}

\subsection{Applications of Doppler Radar Technologies}

Although the primary applications of Doppler radar technologies have been storm tracking, perimeter detection, and vehicle speed measurement (police speed guns), the same principle of detecting a frequency shift in a reflected/received radar signal can be used to detect small physiological movements non-obtrusively. This has many applications in both clinical and non-clinical environments including:

- Search and rescue operations where people are people are trapped under snow (avalanches), earth, soil, and debris (earthquakes and other natural disasters) [34], [35], [36].

- In pediatric clinics to monitor Sudden Infant Death Syndrome (SIDS) [35].

- In sleep studies as a cost-effective solution for long-term monitoring of sleep apnea which does not affect or alter the daily/nightly rhythms of patients [34], [35].

- Assessment of key parameters relating to cardiopulmonary activity, and as a potential substitute to conventional respiratory monitors in hospitals [34], [35].

The remainder of this section reviews work done on the application of Doppler radar to vital-signs monitoring and also discusses advancements in technology and signal processing for the purposes of this application [37] - [51]. 


\subsubsection{Vital Signs Monitoring with Doppler Radar}

\subsubsection{Continuous Wave Radars}

A primary goal of sleep studies is to monitor the breathing rate and heart rate of a patient in order to detect potential sleep disorders. Ideally, these data should be monitored using nonobtrusive, contactless methods. Researchers in [37] sought to improve the monitoring of these vital signs and developed a $24 \mathrm{GHz}$ medical $\mathrm{CW}$ radar. This novel method applied optical interferometry to a six-port receiver providing accurate detection and minimized hardware complexity [37]. Subjects were monitored lying down with the radar positioned on a table above them (the height was not specified). Following signal processing, the radar output signal was converted into the frequency domain using the Fast Fourier Transform (FFT). Results showed that the radar could accurately detect the breathing and heartbeat of a person, even at a distance of several meters.

In [38], researchers performed similar experiments using dual $24 \mathrm{GHz}$ radars installed between the bed bases and mattresses of elderly patients (with and without breathing disorders) to screen for Sleep Apnea-Hypopnoea Syndrome (SAHS). Their method was based on estimating the amplitude probability distribution of the microwave radar respiration signals. Results showed that the estimated amplitude probability can be used to screen for SAHS. Patients with breathing disorders were found to have a higher probability of apnea/hypopnoea occurrence than patients without breathing disorders, as expected.

A 2.4 GHz CW wireless bio-radar sensor was designed and implemented in [39]. Because of its prevalence in wireless communication systems, researchers used signal-tonoise ratio (SNR) analysis as a key design parameter and also took into consideration 
signal power attenuation in the human body. To verify the performance of the bio-radar sensor, the output signal was compared to reference ECG and reference respiration signals (via chest strap) which were collected simultaneously from subjects asked to breathe normally while sitting down. The extracted respiration signals were similar to the reference signals, both having the same respiration rate but being slightly out of phase with each other. The heartbeat signals from the sensor and the ECG differed in terms of peak-to-peak interval but overall, it was concluded that the bio-radar sensor could detect and measure breathing and heartbeat accurately.

$\mathrm{CW}$ radars have also been tested for monitoring the elderly, and those with less mobility, or long term health conditions, in their households. In [40], [41], a pervasive health care system was presented in which non-contact vital signs sensors were embedded in wheelchairs, beds, and walls, and the system was evaluated using several antenna types. Test were conducted on healthy volunteers who were seated and asked to breathe normally. The ability of the system to continuously monitor breathing and heart rates showed the viability of using such sensors for pervasive health care monitoring.

\subsubsection{Ultra-Wideband Radars}

Preliminary work was conducted in [42] using short-distance UWB radars for the medical application of measuring a patient's heart activity and breathing. Advantages of using UWB radars as a measurement method compared to $\mathrm{CW}$ radars were listed and included: smaller device size, decreased electromagnetic radiation level, and increased resolution, which permits the measurement of heart and thorax motion separately. Tests in which subjects were standing still compared the radar output signal with ECG signal data as well as a medical cardiograph to quantify the accuracy of the radar measurements. The 
correlation coefficient was calculated to be approximately 0.91 with an average error of less than $3 \%$.

Testing was also conducted to validate the radar's ability to detect a live person concealed behind a non-transparent barrier. Subjects on the opposite side of a brick wall 0.45 meters thick were asked to alternate between standing still and moving around the room [42]. A person moving and remaining stationary could be clearly seen from the time domain signals (large amplitude signals during motion and smaller periodic breathing signals while person was standing) and a breathing rate was evident in the frequency domain analysis [42].

Previous work with UWB radars focused on detecting cardiorespiratory signals and monitoring the movement of a single subject in a room, but because UWB radars have better spatial resolution than $\mathrm{CW}$ radars, they should be able to track multiple subjects in an environment. A low cost UWB radar sensor which could simultaneously localize and detect breathing of a human subject was presented in [43]. Three separate tests were conducted: detecting a single human subject, detecting two human subjects at different distances (approximately 1.5 meters down range of one another and 0.7 meters off to the side), and detecting two human at the same distance (both 2.5 metres down range of the radar and separated by 0.8 meters) [43]. In all the tests, the radar was able to accurately locate the position of the subjects and measure their breathing, which shows the viability of this method for vital signs detection in hospital monitoring and especially, in rescue operations.

Another potential application of UWB radars is lung cancer radiotherapy as presented in [44]. In lung cancer radiotherapy, respiratory gating and tumour tracking help to deliver 
radiation doses straight to a tumour and minimize the irradiation of surrounding tissues. Since lung tumour motion is induced by respiratory motion (which is closely related to the external chest wall motion and abdominal surface motion), two miniature $2.4 \mathrm{GHz}$ UWB radars were used to simultaneously monitor and measure the thorax and abdominal motions of subjects who were lying down. These demodulated signals were processed and combined to generate gating signals (amplitude and phase gating) to track tumour motion. Experimental analysis proved the reliability of using UWB radar for respiratory gating and accurate tumour tracking.

\subsubsection{Advances in Technology and Signal Processing}

Unwanted body movements can impact the signal reflected off a person and the detection accuracy. Mathematical modelling of the Doppler breathing signal can help develop and improve vital sign monitoring algorithms, such that they can predict a vital sign (radar output) signal being collected in real time, and reduce noise [45]. A mathematical model of the breathing signal was created in [45], comprised of three essential signals: 1) the displacement of the chest due to breathing (represented by four partial functions), 2) the displacement of the chest due to heart motion (considered as a sinusoidal wave), and 3) additive noise (white Gaussian noise). The results from the model were compared to reference data obtained from a $24 \mathrm{GHz} \mathrm{CW}$ Doppler radar and a respiratory transducer belt, and showed good agreement in both the time and frequency domains [45].

One element missing from above-mentioned model is the presence of random body movement. When a person is awake and lying down, they can remain still however, when that person is asleep, sitting, or standing, they are more likely to move randomly or sway from side to side. In [46], complex signal demodulation was studied with the goal of 
eliminating false alarms due to random body movement in monitoring systems. Simulated results matched up with experimental results obtained from $7 \mathrm{GHz}$ portable radars demonstrating that signal demodulation can be used for random body movement cancellation [46].

Researchers in [47] considered applications where moving radar systems may be used for mobile non-contact vital sign monitoring (e.g. unmanned vehicles as first responders). The movement of the radar as opposed to the subject under observation presents different and unfamiliar challenges with regards to vital sign detection. It was speculated that if the radar motion could be accurately tracked then the accuracy of vital sign measurements should improve [47]. Researchers tracked the movement of a $2.4 \mathrm{GHz}$ radar unit using infrared tracking, and applied both Butterworth IIR and adaptive filters for movement cancellation [47]. The filtered signals were compared to breathing signals using a respiratory band and were found to match up well.

Additional research has investigated alternative signal processing techniques to remove noise as well as improve sensitivity [48], [49], [50]. Wavelet transform (now often used in data compression, image processing, and electrocardiogram analysis) has been applied to $\mathrm{CW}$ radars to accurately detect heart rate without the need for filtering [49]. Efforts have also been made to develop more cost efficient and power efficient radar sensors using System-On-Chip RF transceivers, which can accurately detect motion under different modes of operation (CW, FMCW, and packet) [51].

\subsection{Summary}

Doppler radar technology is capable of measuring the radial velocity of a moving object. Although Doppler radar has been primarily used for weather tracking and speed 
detection, numerous studies have shown the viability of using continuous wave and ultrawideband Doppler radars to detect and monitor breathing and heartbeat in clinical and non-clinical environments. The accuracy of these methods have been confirmed using more conventional methods of breathing and heartbeat measurement (RIP bands and ECG respectively). Additionally, the low power output of these radars means that electromagnetic emissions can meet safety guidelines and have no adverse effect on people under observation. 


\section{Chapter: Experimental Setup and Equipment}

This chapter provides information regarding the experimental setup and equipment used for data collection and testing.

\subsection{Equipment}

\subsubsection{Doppler Radar System}

The radar units in this thesis are pseudorandom spread spectrum CW Doppler radars. The use of these radars was a matter of convenience in that the choice of the radar was not optimized, but rather, it was supplied by K\&G Spectrum. The main advantage of the $\mathrm{K} \& \mathrm{G}$ radars is that they use a $24.125 \mathrm{GHz}$ operational frequency, which is consistent with previous investigations. Additionally, this operational frequency is an Instrument Scientific and Measurement (ISM) approved FCC band. The bandwidth of the radar is $\pm 200 \mathrm{MHz}$, which is wide enough to prevent the occurrence of dead RF spots within a cell, but narrow enough to be attenuated by a wall. Three different Doppler radar units (provided by K\&G Spectrum) were used during the different rounds of testing. Different radar units were used based on their availability (the radar units were shared between Carleton University, the University of Ottawa, and $K \& G$ Spectrum) and because $K \& G$ Spectrum occasionally updated the software and firmware.

The radars have a range up to 200 meters. Each radar's range is split into smaller "zones"; their size based on the radar's range resolution. The radars have two operating modes: 1) Scan, and 2) Dwell mode. In Scan mode, the radar collects data for all the channels (zones) it can collect from (10 for the case of the SR12003 radar unit) and samples the radar output signal at a rate of $147 \mathrm{~Hz}$. In Dwell mode, the radar collects data 
from one channel/zone and the radar output signal is sampled at a higher rate of $1471 \mathrm{~Hz}$.

The specifications of the K\&G radars are listed in Table 3.1.

Table 3.1 Doppler radar units used in this research.

\begin{tabular}{|c|c|c|c|c|}
\hline $\begin{array}{c}\text { Radar } \\
\text { Model }\end{array}$ & $\begin{array}{c}\text { Beam } \\
\text { Width }\end{array}$ & $\begin{array}{c}\text { Range } \\
\text { Resolution (m) }\end{array}$ & $\begin{array}{c}\text { Operating } \\
\text { Frequency (GHz) }\end{array}$ & $\begin{array}{c}\text { Number of } \\
\text { Zones }\end{array}$ \\
\hline SR12005 & $120^{\circ} \times 5^{\circ}$ & 3.00 & 24.125 & 50 \\
\hline SR12003 & $120^{\circ} \times 5^{\circ}$ & 0.75 or 3.00 & 24.125 & 10 \\
\hline SR2003 & $20^{\circ} \times 3^{\circ}$ & 0.75 & 24.125 & 10 \\
\hline
\end{tabular}

Figure 3.1 shows the SR12003 radar in its vertical and horizontal orientations. In the vertical orientation, the beam spreads more in the horizontal direction, as opposed to the horizontal orientation of the radar, where the beam spreads more in the vertical direction.

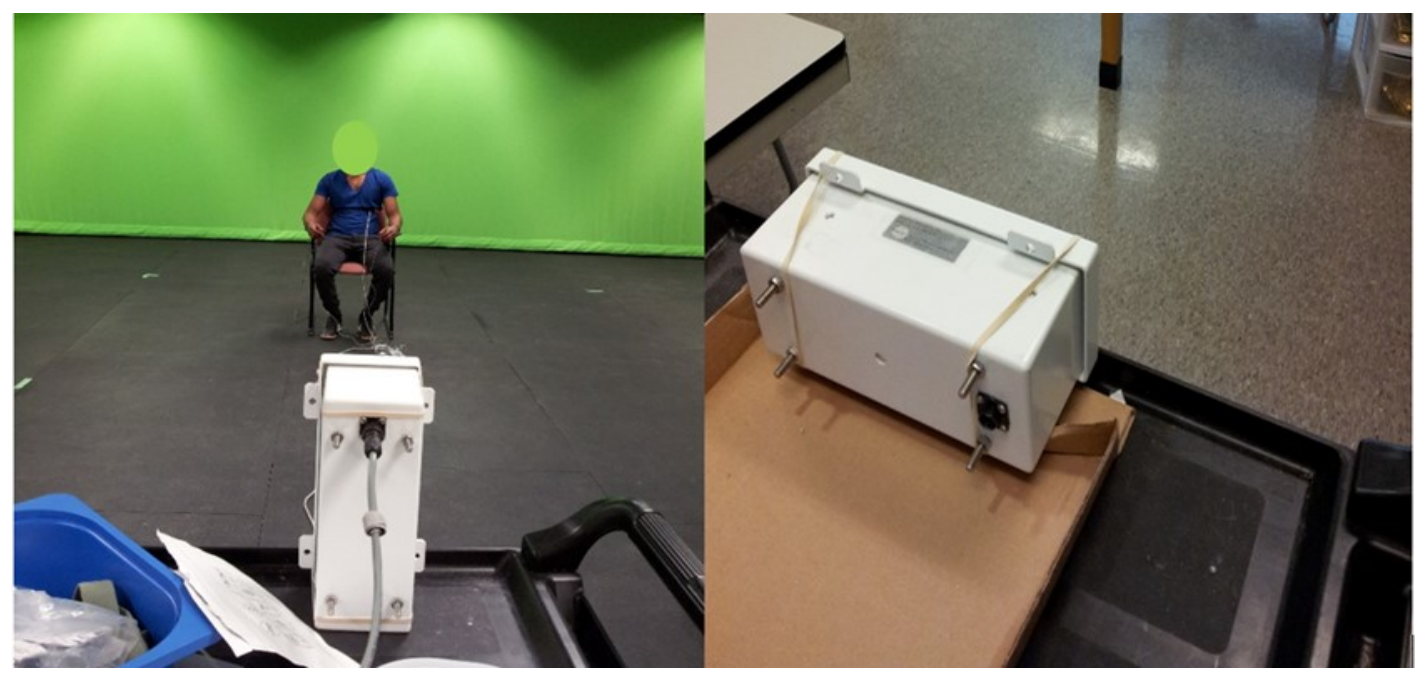

Figure 3.1 The SR12003 radar unit in its vertical orientation (left) and horizontal orientation (right).

The Doppler radar units were all configured as shown in Figure 3.2. 


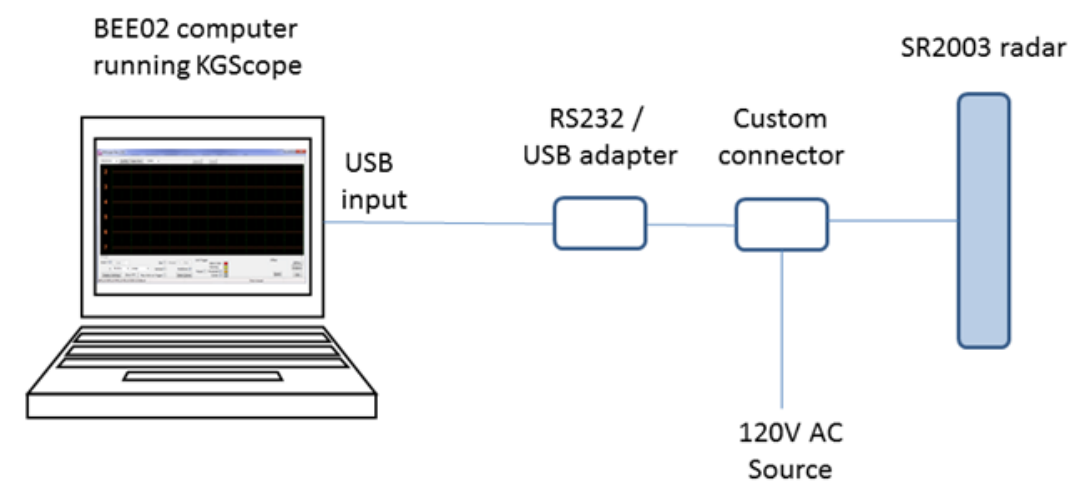

Figure 3.2 Doppler radar system setup.

KGScope software (version 2.52) was used to control the radar unit and record data. The KGScope software configuration for the data collection differed for the different rounds of testing. The specific configurations parameters for each round of testing will be explained in later sections. Figure 3.3 shows the configuration setup screen. For signal analysis, data collected using the KGScope software was converted to CommaSeparated-Values (CSV) format using the GUI-based KGtoCSV tool (version 1.00) and then imported into Matlab. It should be noted that the time scale in the KGScope software was incorrect but since the data were converted and analysed in Matlab, this had no effect on the results. K\&G Spectrum plans to resolve this time scale issue in a future release of the software. 


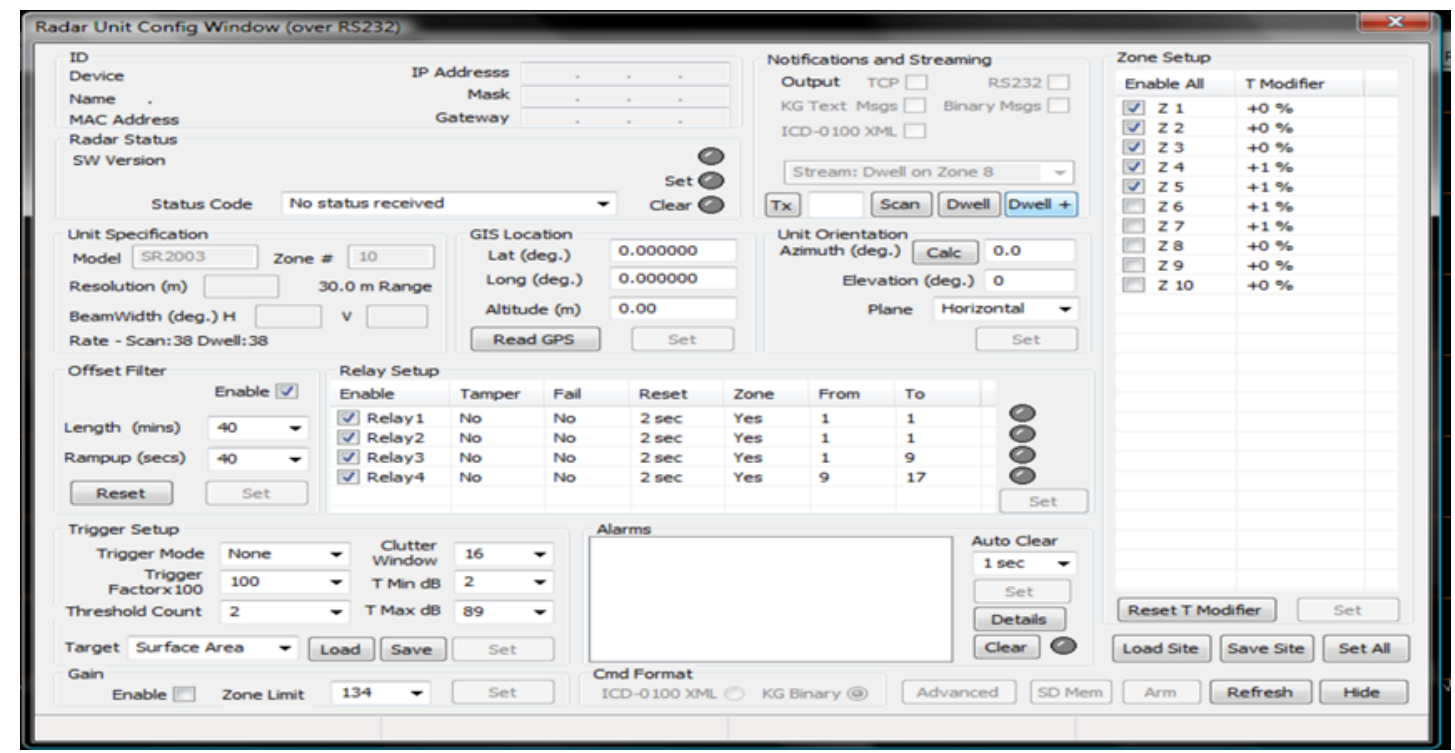

Figure 3.3 KGScope radar configuration setup screen.

\subsubsection{Respiratory Inductance Plethysmography (RIP) Band}

In some tests, subjects wore a Respiration Inductance Plethysmography (RIP) band (model 0528 Piezo Respiratory Effort Sensor; Braebon Medical Corporation; Kanata, ON, Canada) which was used as a gold standard for respiration activity that the Doppler radar could be compared against. The RIP band (Figure 3.4) was placed mid-chest around the subject slightly below the armpits.

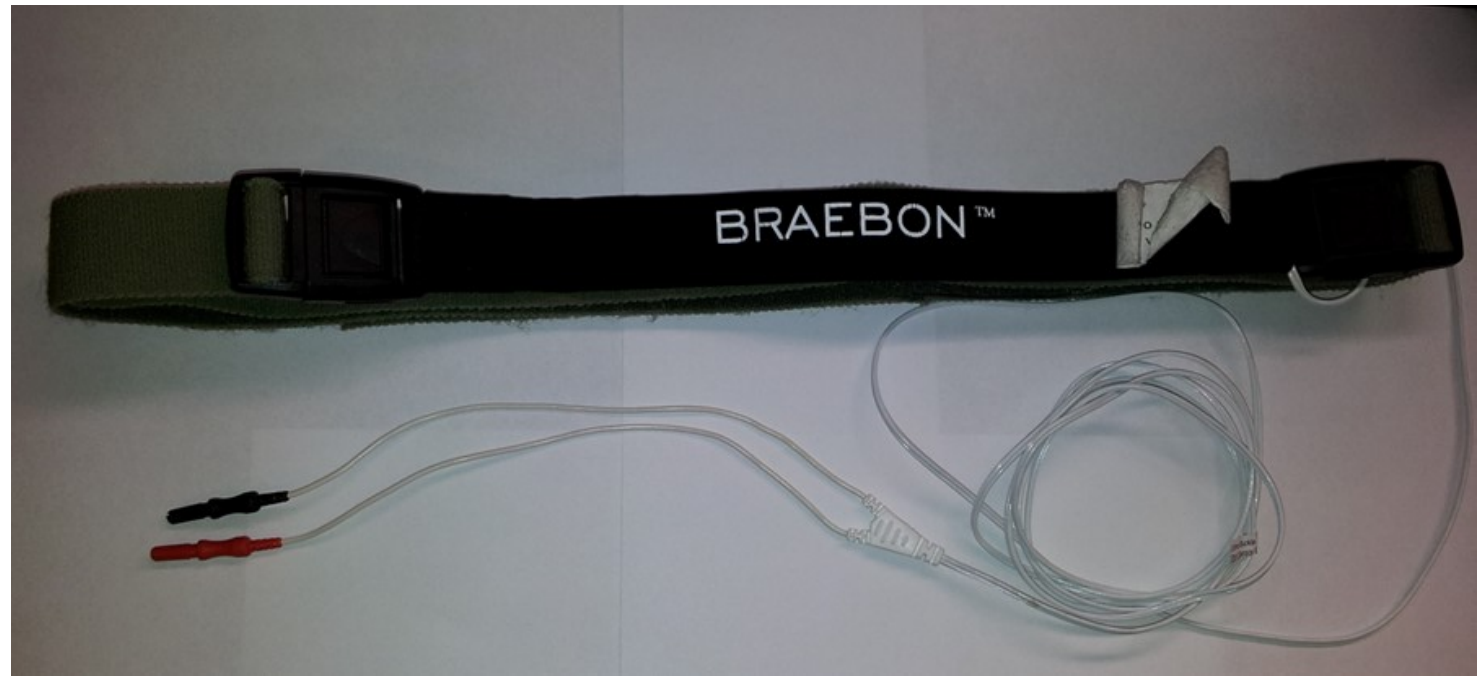

Figure 3.4 RIP band for respiration data collection. 


\subsubsection{Electrocardiogram (ECG) Electrodes}

Some tests acquired the ECG from subjects, which was used as a gold standard for heartbeat activity that the Doppler radar could be compared against. In these tests, conventional $\mathrm{Ag} / \mathrm{AgCl}$ surface electrodes (model MVAP-II; MVAP Medical Supplies; Newbury Park, CA, USA; Figure 3.5) were used. A Lead I ECG was acquired. Subjects had the electrodes placed on each wrist on the ventral side of the arm, and a ground electrode on the left ankle. Typically, in a standard Lead I ECG, the ground is the right leg, so the placement used in this thesis is not a standard Lead I ECG.

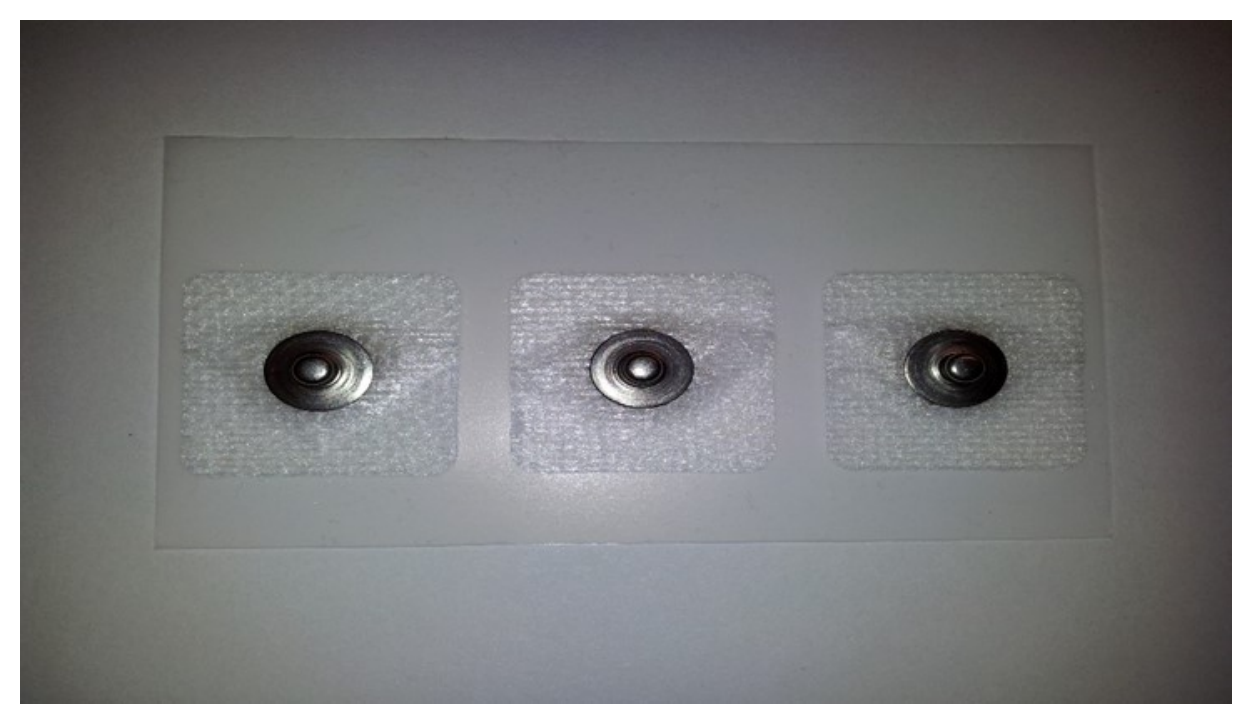

Figure 3.5 Ag/AgCl electrodes for ECG data collection.

\subsubsection{Bioinstrumentation Amplifier and Data Acquisition Unit (DAQ)}

The ECG electrodes and RIP band were connected to an electrode connector box (Model F-15EB/B2; Grass Telefactor; Warwick, RI, USA). The connector box was connected to the Grass Telefactor M15LT amplifier system, which housed programmable quad amplifiers (model 15A54; Grass Telefactor; Warwick, RI, USA). The ECG signal was amplified with a gain of 2000 and a bandwidth of $1 \mathrm{~Hz}$ to $100 \mathrm{~Hz}$. The RIP band signal was amplified with a gain of 200 and a bandwidth of $0.1 \mathrm{~Hz}$ to $30 \mathrm{~Hz}$. The signals were 
sampled at $1000 \mathrm{~Hz}$, using a 16-bit analog-to-digital converter (model USB-6216; National Instruments; Austin, TX, USA) set to a $\pm 5 \mathrm{~V}$ input range.

\subsubsection{Vicon Motion Capture System}

The Vicon motion capture system at Carleton University’s Motion Capture Lab (Azrieli Pavilion Room 236) was used to collect data on the breathing motion of the upper body. The system is comprised of three main components: 1) the cameras, 2) the room, and 3) the markers.

The six cameras in the Motion Capture Lab were Vicon MX40 cameras. Each camera has red LED lights that circle the front of the camera. These lights make up the camera's strobe, which emits infrared light to illuminate the reflective markers. Additionally, each camera has an onboard processor, which collects information from the sensor, processes it, and sends it in the form of compressed 2D data (containing only the grayscale reflections of the markers) to the network which links the cameras together, and then onto a program called Tarsus [52].

The floorplan of the Motion Capture Lab is shown in Figure 3.6. The dimensions of the room are $27^{\prime}$ x $38^{\prime}$ [52]. Since at least 3 cameras are required to accurately track a reflective marker, the subject must be located within the Field-of-View (FOV) of at least 3 cameras. All reflective surfaces in the room have been removed or covered, such that the cameras should only pick up the reflective markers within their FOV. Additionally, blackout curtains have been used on the windows in order to block out any infra-red light from the sun. 


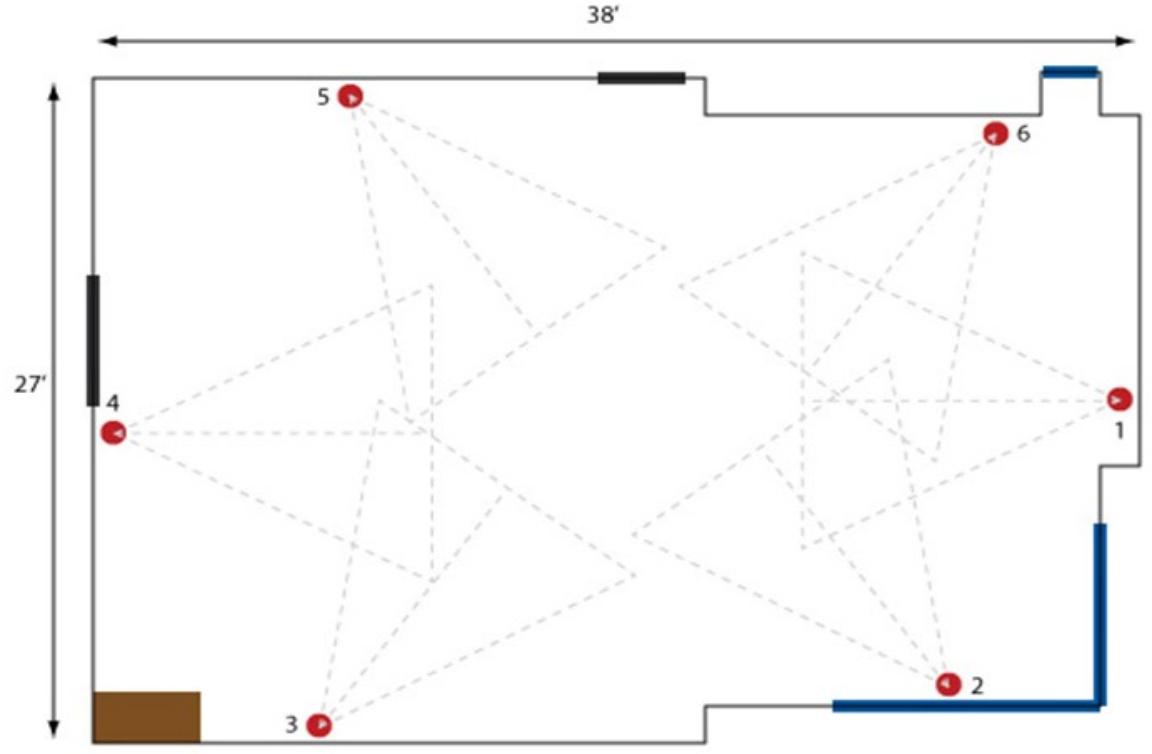

Figure 3.6 Floorplan of the Motion Capture Lab at Carleton University (modified from [52]).

The reflective markers (pictured in Figure 3.7) used were spheres (14 $\mathrm{mm}$ in diameter) with retroreflective tape on them. These markers had removable bases which were attached to subjects using two-sided tape.

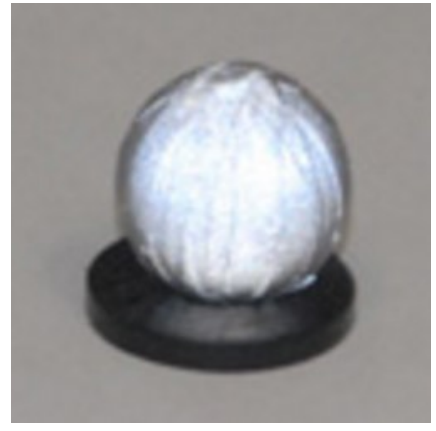

Figure $3.714 \mathrm{~mm}$ reflective marker used in Vicon motion capture.

\subsubsection{Signal Processing}

Data were processed offline using Matlab (version R2014b; Mathworks; Natick, MA, USA). 


\section{Chapter: In-Phase Quadrature (I/Q) Testing}

\subsection{Introduction}

Doppler radars transmit signals which are reflected off a target and are then demodulated in the receiver [53], [54]. According to Doppler theory, a target with a time-varying position but a net zero velocity (e.g. a stationary human body), will reflect the signal with its phase modulated proportionally to the time-varying target position [53], [55]. The breathing and cardiac activity in a stationary human body represent two sources of independent time-varying sources of motion with zero net velocity [54]. The largest reflection of incident radio frequency power occurs at the body surface (chest). Demodulating the phase information will provide information on the position of the chest from which, data on the movement and rate due to breathing and heart beat can be extracted [53], [55].

Problems arise when the same source in the radar is used for transmitting and receiving, relating to the periodic phase relationship between the received signal and the local oscillator (a clock responsible for timing of all radar operations) [23], [53], [54]. The received signal is mixed with the local oscillator signal, and the resulting baseband signal (see equation 4.1) is a function of residual phase noise and a constant phase shift, which is dependent on the nominal distance to the target [53], [55].

$$
\begin{gathered}
B(t)=\cos \left[\theta+\frac{4 \pi x(t)}{\lambda}+\Delta \emptyset(t)\right] \quad \text { (4.1) } \\
x(t)=\text { target displacement } \quad \phi(t)=\text { phase noise } \quad \theta=\text { phase shift }
\end{gathered}
$$

When the phase shift is an odd multiple of $\pi / 2$, the optimum phase-demodulation sensitivity is achieved and the baseband is linearly proportional to the periodic chest displacement summed with the residual phase noise (see equation 4.2) [53], [55]. 


$$
B(t) \approx \frac{4 \pi x(t)}{\lambda}+\Delta \emptyset(t)
$$

When the phase shift is an integer multiple of $\pi$, the null-demodulation sensitivity is decreased and the baseband output (see equation 4.3) is no longer proportional to the time-varying displacement [53], [55].

$$
B(t) \approx 1-\left[\frac{4 \pi x(t)}{\lambda}+\Delta \emptyset(t)\right]^{2}
$$

When the LO and received signals are either in-phase or 180 degrees out-of-phase, "optimum" and "null" points occur periodically with a target distance every quarter wavelength $(\lambda / 4)$ from the radar [53], [54]. The wavelength is calculated as follows:

$$
\begin{gathered}
\lambda=c / f_{c} \\
c=\text { speed of light }=3 \times 10^{8} \frac{\mathrm{m}}{\mathrm{s}} \quad \text { and } f_{c}=\text { radar carrier frequency }(\mathrm{Hz})
\end{gathered}
$$

At null points, the signal power is low. Quadrature (or phase) detectors have two receiver channels which separate the received signal into in-phase (I) and quadrature (Q) channels which are 90 degrees out-of-phase from each other [23], [53], [55]. Quadrature detectors ensure that the signals will never be zero in both channels (at least one channel signal is not in the null point) [23], [53].

$$
I=A \cos \emptyset \quad \text { (4.5) } \quad Q=A \sin \emptyset
$$

Using the above equations, when the phase shift is an odd multiple of 90 degrees, the baseband signal of the I channel will be at an optimum, while the Q channel will be at a null point. Conversely, when the phase shift is an integer multiple of 180 degrees, the baseband signal of the Q channel will be at an optimum, and the I channel will be at a null point [54]. 
Testing was done to investigate if there was a noticeable I/Q effect during data collection. The radars supplied by $K \& G$ spectrum do not currently have quadrature detectors, making them susceptible to these nulls. This is only a concern if the motion of interest is also small, since a large motion would move the detector in and out of the null point. The radar unit has a carrier frequency of $24.125 \mathrm{GHz}$, and therefore, has a wavelength of approximately $12.4 \mathrm{~mm}$. This means that the distance between an optimum point and a null point is expected to be $3.1 \mathrm{~mm}$ (a quarter wavelength of the $12.4 \mathrm{~mm}$ wavelength). It is unclear whether the breathing motion would be large enough to move the detector in and out of the null point. Results from this testing can inform the need to include quadrature detectors in future radar units. The addition of quadrature detectors will increase the cost and complexity of design and implementation of the radar units; unless it is necessary, it should be avoided.

\subsection{Methodology}

The SR12005 radar unit was used for the testing described in this chapter. The radar's operating frequency of $24.125 \mathrm{GHz}$ implies a signal wavelength of approximately 12.4 $\mathrm{mm}$.

\subsubsection{Experimental Setup}

The testing was conducted in a Carleton University classroom (University Centre Room 280 ), approximately 28 " by 47 ," where all the chairs and tables were moved to the sides of the classroom to minimize any potential interference and sources of reflection (see Figure 4.1). The subject was seated in a chair with a backrest 7 feet (measured from the front legs of the chair) from the SR12005 radar unit, optimizing recording in Zone 2; optimized by shifting the radar unit a few centimetres towards and away from the seated 
subject until the signal amplitude appeared to be at its maximum. The radar was set to Dwell mode and oriented vertically so that the beam was spread across the width of the classroom (see Figure 4.2).

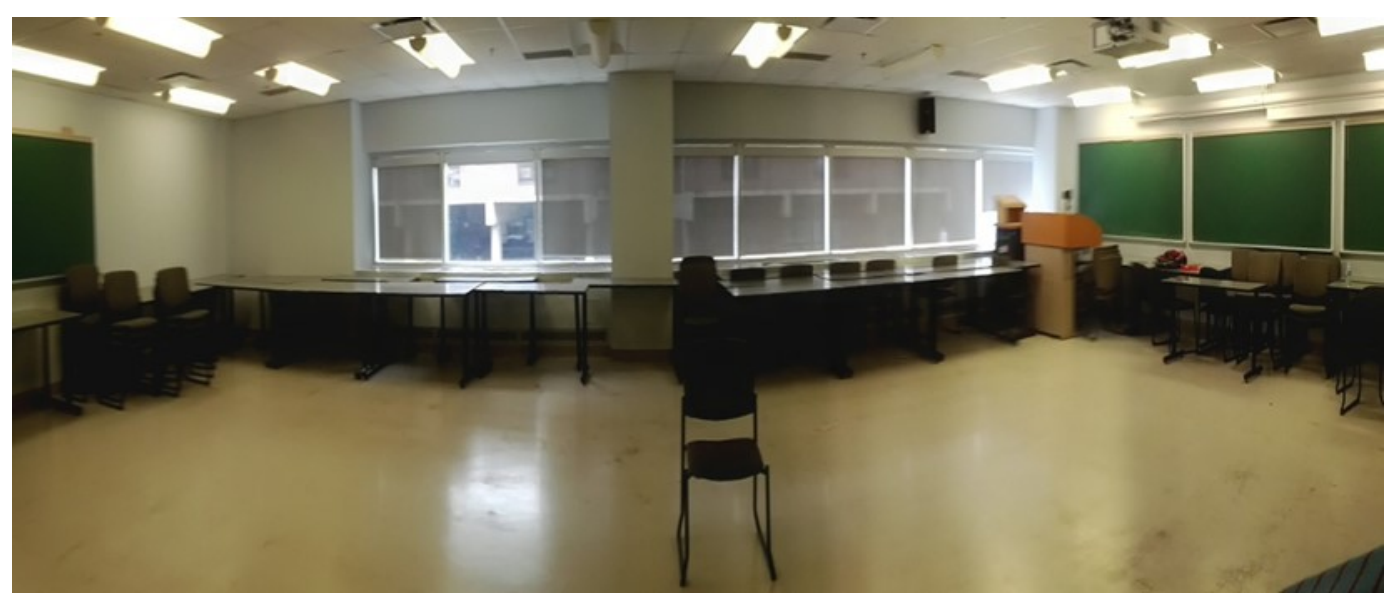

Figure 4.1 Picture of measurement environment facing away from the SR12005 radar unit.

Subject

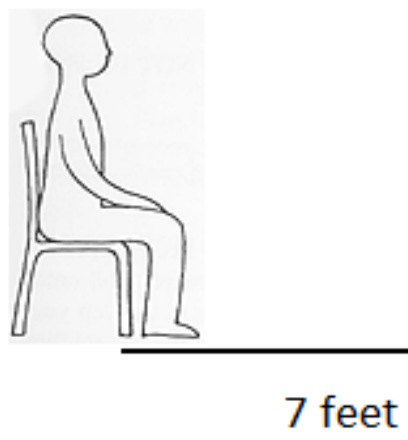

SR12005 in vertical orientation

Figure 4.2 Subject position and experimental setup of radar unit.

\subsubsection{Experimental Procedure}

A wooden board with millimeter markings was placed beneath the radar to accurately move the radar by small increments. For the first recording (i.e., the optimized position), the radar was placed on a marking which was deemed position $0 \mathrm{~mm}$, and the subject's breathing was recorded for 30 seconds (zones 1-5 were monitored in scan mode). Subsequent recordings were conducted moving the radar in $1 \mathrm{~mm}$ increments, with the 
subject's breathing recorded for 30 seconds in each recording. The radar was moved in 1 $\mathrm{mm}$ increments backward (away from the subject) to a relative position of $-7 \mathrm{~mm}$. Then the radar was moved in $1 \mathrm{~mm}$ increments forward (towards the subject) to a relative position of $7 \mathrm{~mm}$. Then the radar was moved in $1 \mathrm{~mm}$ increments backward to a relative position of $0 \mathrm{~mm}$. The produced a total of 31 recordings, with each position measured twice, except for position $0 \mathrm{~mm}$, which was repeated three times.

\subsection{Results}

For each of the 31 recordings, the biased variance estimate for the zone 2 data was calculated to determine the AC power of the radar output signal (see equation 4.7). The radar output signal amplitude is represented by $R, N$ refers to the number of samples, and $\mu$ is the mean of the radar output signal amplitude.

$$
V=\frac{1}{N} \sum_{i=1}^{N}\left|R_{i}-\mu\right|^{2}
$$

The zone signal power in decibels was then plotted against the position of the radar (relative to position $0 \mathrm{~mm}$ ). Figure 4.3 shows the scatter plots of Zone 2's power plotted against the position of the radar. The data set "AC Power" is made up of the AC power values calculated for each test. There are multiple data points at the same distance markers because recordings were taken twice at each position (three at Position 0). The data set "Average AC Power" is made up of the average of each position's power values.

Figure 4.3 shows maximum power values in the range $-1 \mathrm{~mm}$ to $1 \mathrm{~mm}$ and minimum power values around $-4 \mathrm{~mm}$ to $-5 \mathrm{~mm}$ and $4 \mathrm{~mm}$ to $5 \mathrm{~mm}$. 


\section{Zone 2 AC Power vs. Radar Position}

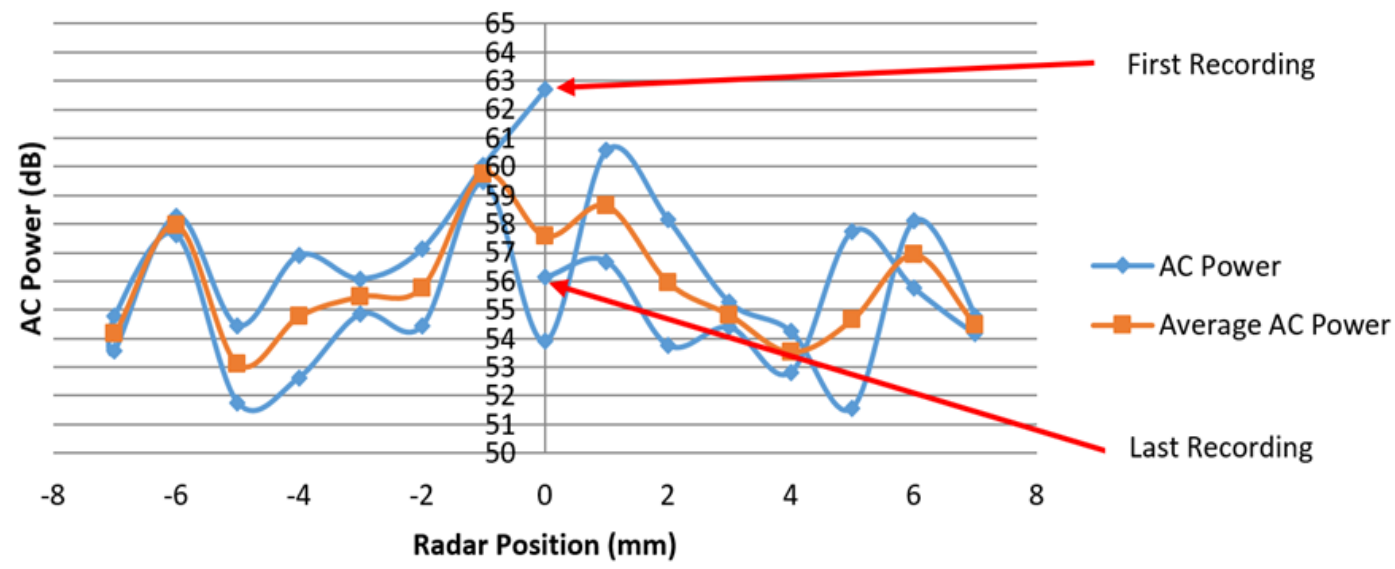

Figure 4.3 Scatterplot of Radar's Zone 2 signal power versus its position relative to Position 0.

\subsection{Discussion}

From the $0 \mathrm{~mm}$ position, the signal power does first decrease and then increases. Null points were observed at $4 \mathrm{~mm}$ and $-5 \mathrm{~mm}$, though they were expected closer to $\pm 3 \mathrm{~mm}$, and optimum points were observed at $\pm 6 \mathrm{~mm}$, as expected. The repeatability was not great in general for each point. For example, at $0 \mathrm{~mm}$, and $5 \mathrm{~mm}$, there is large variability in the $\mathrm{AC}$ power.

Noise resulting from subject motion could confound results. There were 31 recordings of 30 seconds each for the I/Q testing, resulting in a very tedious data collection process. While the radar was moved incrementally by $1 \mathrm{~mm}$, it is unlikely that the subject maintained sub-mm accuracy in their position relative to the radar throughout the collection process, which lasted longer than 20 minutes (there was time required to reposition the radar between recordings as well.

Despite the noise, it seems there is an I/Q effect since the I/Q trend is still visible, with the signal power oscillating in a pattern that appears consistent with the radar wavelength. Despite this, it is not clear whether I/Q demodulation features are necessary in future 
radar units. There is a max difference of approximately $9 \mathrm{~dB}$ between the max and min magnitude points. With every $3 \mathrm{~dB}$ loss, the signal power is halved, so a $9 \mathrm{~dB}$ difference results in the null points having roughly one-eighth the signal power compared to the optimum points. Although, the signal-to-noise ratio (SNR) was noticeably poorer at the null points, the breathing signal was still measurable in all positions. Additionally, it is unlikely that a person would remain in a "null" for an extended period of time. For the target application (i.e., correctional institutions), multiple radar units would likely be used to allow localization of targets within a room.

With such a multi-radar system, the likelihood of a target/person being in a "null" for two radars for an extended amount of time would be much lower than for a single radar system. Though adding I/Q demodulation features would avoid the null point problem altogether, it would also increase the cost and complexity of the radar system.

\subsection{Conclusion}

Testing was conducted to determine if future radar units would benefit from I/Q demodulation features. Results do confirm the presents of null points where the AC power and SNR were poorer; however, considering the increased cost and complexity, which would come with the addition of this technology, it is difficult to say with absolute certainty that I/Q demodulation is necessary for future radar units. For the given application of vital-signs monitoring of inmates, I/Q demodulation is probably not essential, especially if there are multiple radar units simultaneously employed. 


\section{Chapter: Breathing Estimation Algorithm}

\subsection{Introduction}

The main focus of this chapter is the implementation and evaluation of a breathing estimation algorithm for a Doppler radar system, which estimates a person's breathing rate from the output signal measured by the radar unit. The breathing estimation algorithm was created with the intent of providing real-time vital signs monitoring, and forms a baseline for future algorithms to be compared against; as such, the estimation algorithm complexity was kept low. It is assumed that the person under observation is not moving (since movement would likely indicate that they were alive). Future work can be done to determine the activity associated with movement.

This chapter describes the breathing estimation algorithm, as well as a performance analysis of the breathing estimation algorithm based on data from human subjects. All testing described in this chapter was reviewed and approved by Carleton University's Research Ethics Committee.

\subsection{Breathing Estimation Algorithm}

Breathing estimation is performed in the frequency domain using a sliding analysis window. Within each analysis window, the absolute magnitude of the Fast Fourier Transform (FFT) analysis is used to generate the magnitude spectrum. The breathing estimation algorithm finds the highest peaks in the magnitude spectrum; the corresponding frequency is considered the breathing rate.

In this chapter, the analysis window was 30 seconds in length, which slides by an increment of 5 seconds; this provides a breathing estimate every 5 seconds. The radars used in this thesis have a sampling frequency of $1471 \mathrm{~Hz}$, which means that there are 
44,130 samples in each analysis window. The FFT has a frequency resolution of 0.033 $\mathrm{Hz}$ making the breathing rate estimation have a resolution of 2 breaths per minute.

All peak frequencies relating to breathing were compared to normal rates for the average healthy adult at rest. The normal breathing rate ranges from 12 to 20 breaths per minute (brpm), or $0.2 \mathrm{~Hz}$ to $0.333 \mathrm{~Hz}$, with rates below $12 \mathrm{brpm}$ or above $25 \mathrm{brpm}$ considered abnormal [56]. Physical exertion/activity however, can increase the breathing rate to 50 breaths per minute, or $0.833 \mathrm{~Hz}$ [56]. Additionally, individuals can intentionally slow their breathing or use techniques like diaphragmatic breathing to lower their breathing rate below 12 breaths per minute (as low as $6-8$ breaths per minute) [57]. During periods of normal breathing (periods of physical activity are not considered normal breathing), this algorithm finds the highest peaks in the magnitude spectrum between 8 brpm and 25 brpm, or $0.133 \mathrm{~Hz}$ to $0.42 \mathrm{~Hz}$.

It is also possible for the Doppler radar to detect the motion associated with the heartbeat and therefore it is possible that one could estimate heart rate. A heart rate estimation algorithm was also implemented, which also operated on the magnitude spectrum of the Doppler radar output signal. The normal heart rate for healthy adults ranges from 60 to 80 beats per minute (bpm), or $1 \mathrm{~Hz}$ to $1.333 \mathrm{~Hz}$ [56]. During periods of breath hold (when there should theoretically be no breathing signal) the algorithm finds the highest peaks in the magnitude spectrum between $50 \mathrm{bpm}$ and $100 \mathrm{bpm}$, or $0.833 \mathrm{~Hz}$ and $1.667 \mathrm{~Hz}$.

\subsection{Methodology}

The breathing estimation algorithm was implemented using Matlab (version R2014b) with the window size and sliding increment between windows as input variables. The window size can be set to analyse longer or shorter segments of data as desired; longer 
windows provide high frequency resolution in the spectrum (and higher breathing rate resolution) but lower time resolution. By varying the overlap between windows, one can adjust how frequent the breathing rate estimate is updated.

Data were collected from human participants to evaluate the breathing estimation algorithm. These data included the Doppler radar output signal, RIP band signal, and the ECG. The RIP band signal was used as a gold standard for the breathing rate, to which the breathing rate estimate from the Doppler radar could be compared against. Since it was also possible to estimate the heart rate using the Doppler radar, the ECG was collected to provide a gold standard for the heart rate to compare against.

\subsubsection{Data Collection}

During preliminary testing, different factors (e.g., distance from radar, radar orientation, etc.) were considered and finalized for the experiments in this chapter. The goal of this data collection was to obtain data as clean and noise free as possible, which could then be used for baseline evaluations of the breathing estimation algorithm. This was done by attempting to position the radar unit directly facing the front of the subject and conducting the tests in an environment which would have as few sources of reflection as possible.

A secondary goal of the testing was to provide the dataset using a well-defined, systematic protocol that future research could use. Maintaining consistency in testing will produce data and results that can be compared to each other.

\subsubsection{Experimental Participants}

Data were collected from 5 male subjects with no history of cardiovascular problems or disorders. Physical data on the subjects can be found in Table 5.1 below. 
Table 5.1 Age, weight, and height data of subjects.

\begin{tabular}{|c|c|c|c|}
\hline Subject (\#) & Age & Weight (kg) & Height (cm) \\
\hline 1 & 23 & 84.0 & 174 \\
\hline 2 & 26 & 88.5 & 176 \\
\hline 3 & 25 & 82.0 & 178 \\
\hline 4 & 24 & 64.0 & 175 \\
\hline 5 & 24 & 88.0 & 187 \\
\hline
\end{tabular}

\subsubsection{Experimental Setup}

The majority of the data collection was conducted using the SR12003 radar unit. With subject \#5, an additional set of data was collected using the SR2003. The SR2003 has the same specifications as the SR12003, except the beam width is smaller at $20^{\circ} \times 3^{\circ}$. (The reader can refer to Section 3.1.1 of the Methodology chapter for additional specifications of these radar units).

Experiments for subjects \#1 to \#4 were conducted in Carleton University's Vicon room (Azrieli Pavilion Room 234; $27^{\prime}$ x $38^{\prime}$ ). It is a wide open space with few sources of reflection. Subjects were placed (sitting in a chair with back support and standing) facing the radar unit which was approximately 4 metres away (Figure 5.1). The exact distance and orientation of the radar were optimized during the first test so that the subjects were centered in zone 8. Tape was placed on the ground to mark the subject's position and ensure that the distance from the radar does not change between tests. The radar was set to Dwell mode and was placed in a horizontal orientation so that the beam spread across the entire subject. Tests were done in both the sitting and standing positions to investigate how posture affects the radar output signal and the radar's ability to pick up a person's breathing and heartbeat. 


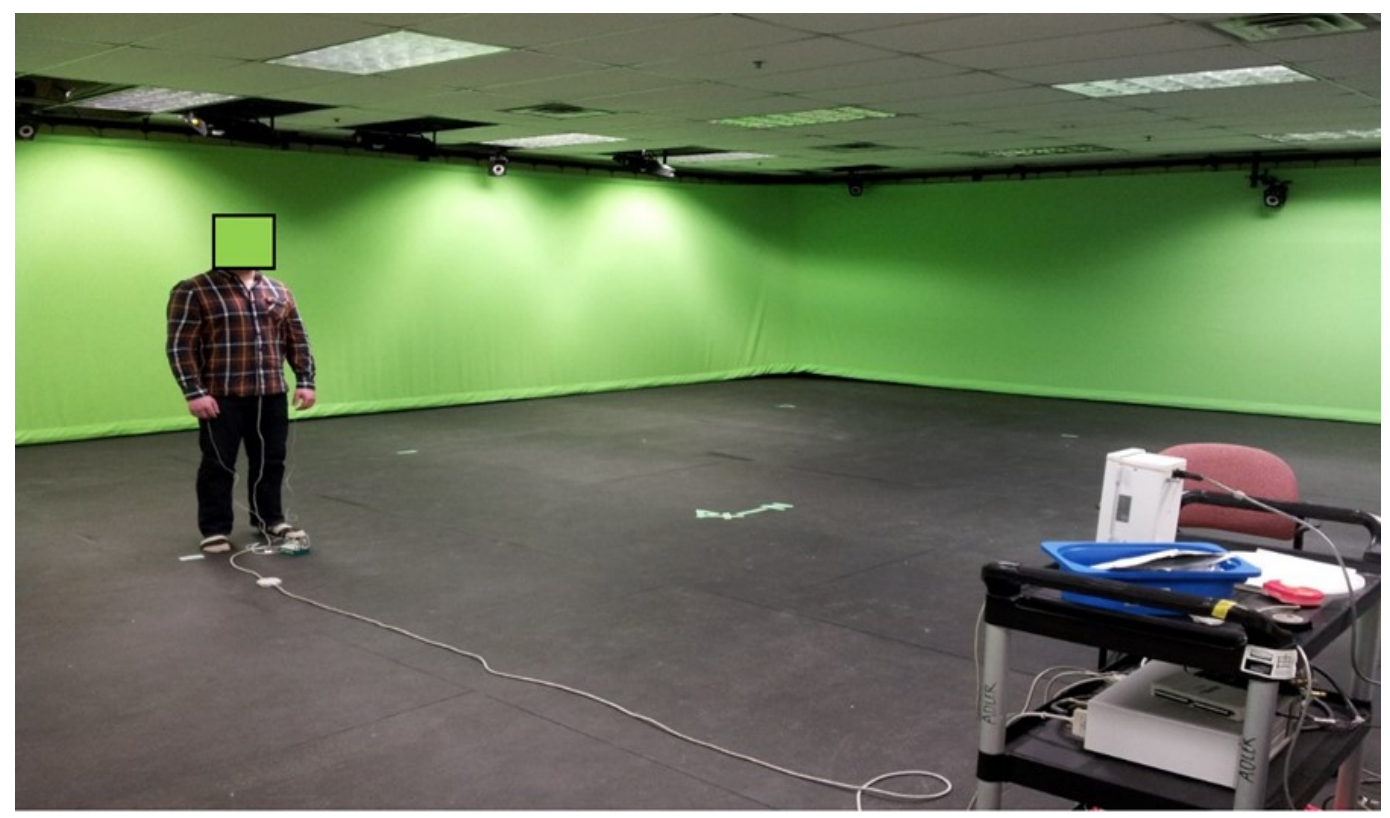

Figure 5.1 View of Vicon room and experimental setup.

With subject \#5, testing was conducted in a Carleton University graduate student lab (Canal Building Room 6207) because of issues with accessing the Vicon room. For these tests, KG Spectrum asked that the SR12003 radar unit be oriented vertically, and that one sitting test be conducted with the SR2003 radar unit. The subject was placed (sitting in a chair with back support and standing) facing the radar unit which was approximately 2.6 metres away for the SR12003 radar tests and 3.1 metres for the SR2003 test. The exact distance and orientation of the radar were optimized during the first test so that the subjects were centered in zone 6 and zone 9 respectively. Table 5.2 highlights the radars used for each test as well as their orientation.

Table 5.2 Radar unit and orientation setup for testing with each subject.

\begin{tabular}{|c|c|c|}
\hline Subject (\#) & Radar Unit Used & Radar Orientation \\
\hline 1 & SR12003 & Horizontal \\
\hline 2 & SR12003 & Horizontal \\
\hline 3 & SR12003 & Horizontal \\
\hline 4 & SR12003 & Horizontal \\
\hline 5 & SR12003 \& SR2003 (one sitting test) & Vertical \\
\hline
\end{tabular}


Electrocardiogram (ECG) data were collected using conventional $\mathrm{Ag} / \mathrm{AgCl}$ electrodes and respiration data were collected using a RIP band. Subjects had $\mathrm{Ag} / \mathrm{AgCl}$ electrodes placed on each wrist and a ground electrode on the left ankle for the ECG data collection. The electrodes were secured using tape to ensure good electrode-skin contact throughout the recording. The RIP band was placed around the subject's thorax (Figure 5.2). To prevent or reduce wire motion stemming from the subject's movement, the ECG and RIP wires were run underneath the subject's clothing and behind their back though this is not shown in Figures 5.1 and 5.2. The ECG electrodes and RIP band were connected to an electrode connector box which was connected to the Grass Telefactor M15LT amplifier system housing programmable quad amplifiers. Signals were sampled at $1000 \mathrm{~Hz}$ using a 16-bit analog-to-digital converter set to a $\pm 5 \mathrm{~V}$ input range. The $\mathrm{ECG}$ signal was amplified with a gain of 2000 and a bandwidth of $1 \mathrm{~Hz}$ to $100 \mathrm{~Hz}$. The RIP band signal was amplified with a gain of 200 and a bandwidth of $0.1 \mathrm{~Hz}$ to $30 \mathrm{~Hz}$. Please refer to section 3.1.4 of the Methodology for more detailed information on the equipment.

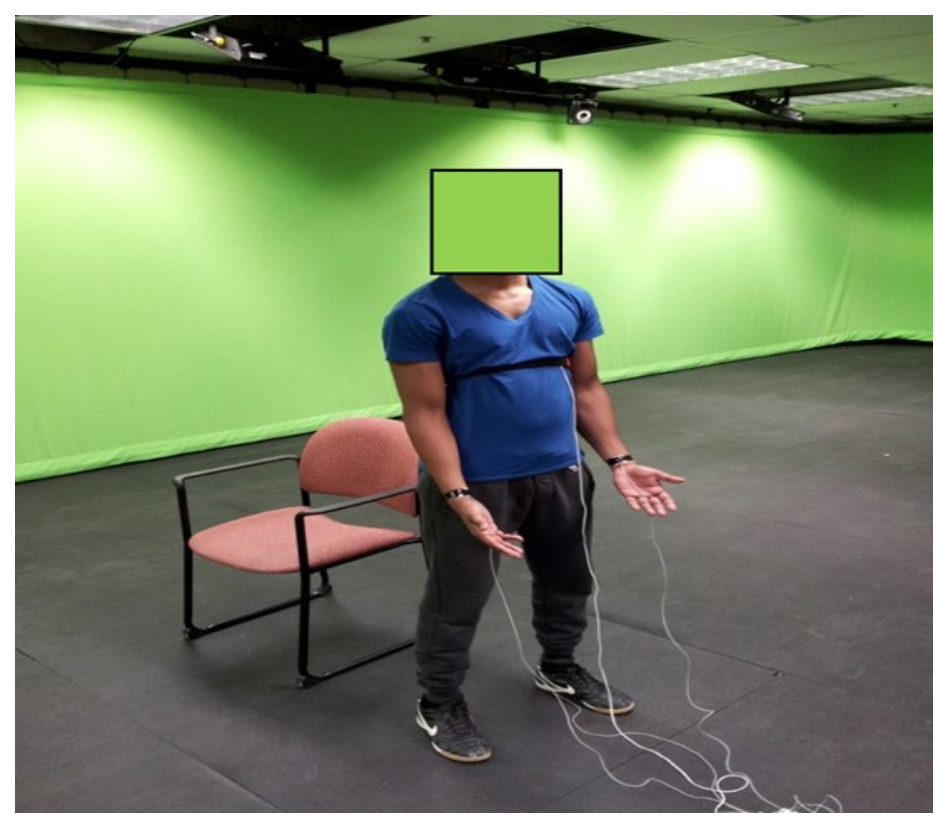

Figure 5.2 ECG and RIP band connections to subject. 


\subsubsection{Experimental Procedure}

Each participant performed six tests, except for subject \#5 who performed seven tests. A single test was broken down into three 5-minute sections as follows:

1. Subjects were asked to remain still and breathe normally.

2. Subjects were asked to perform jumping jacks for 30 seconds to increase their heart rate and breathing rate. For the remaining 4 minutes and 30 seconds, subjects were asked to remain still, and let their breathing and heart rate return to normal.

3. Subjects were asked to remain still, hold their breath for as long as possible/comfortable and then continue breathing, allowing their breathing to return to normal. It was stressed to the subjects that they remain as still as possible during the breath hold.

The period of physical activity was expected to provide noisy data, where any breathing estimate would be unreliable. The change from an elevated breathing and heart rate to baseline levels would help with testing breathing rate estimation by providing a range of breathing rates. The breath hold and recovery section would also help with the performance analysis of the breathing rate estimation algorithm, and would help determine whether a heart rate can be estimated from the radar output signal.

Subjects performed this test in both standing and sitting positions. Three repetitions were done in each posture for a total of six tests per subject. At the beginning and end of each test, the subject was asked to make a 'jolting' motion (e.g., quickly standing up and sitting back down when in the sitting posture, quickly crouching/squatting and standing back up when in the standing posture). This motion was visible on the radar, RIP band, 
and ECG signals allowing the radar output signal to be time aligned with the RIP band and ECG signals. Subject \#5 completed a seventh test in the sitting position using the SR2003 radar unit.

Prior to breathing rate estimation, pre-processing was done on the radar output signal to remove noise. The output signal was passed through a second order low pass Butterworth filter with a cut-off frequency of $5 \mathrm{~Hz}$ which removed $60 \mathrm{~Hz}$ power line interference along with other high frequency noise. Baseline wander was also removed from the signal using a second order high pass Butterworth filter with a cut-off frequency of 0.05 Hz. The RIP band and ECG signals were resampled at $1471 \mathrm{~Hz}$ to match the sampling frequency of the radar.

\subsubsection{Estimation of Breathing and Heart Rate}

Following the signal processing, the breathing estimation algorithm (refer to Section 5.2) was applied to the signals. SR12003 and SR2003 radar, RIP band, and ECG signal segments were analysed in the frequency domain. Magnitude spectrums were computed for each signal and its corresponding segments. From the magnitude spectrums, the primary peaks were identified. The peaks in the radar and RIP band signals were compared to evaluate the accuracy of the breathing estimate algorithm. The breathing rate estimates calculated from the primary peak locations of the RIP band signal magnitude spectrums were manually verified as accurate through visual inspection of the time domain RIP band signal (this meant checking to see that the frequency of peaks within one analysis window equated to the peak frequency in the magnitude spectrums). The peaks in the radar and ECG signals were compared to help determine if the radar is capable of detecting the small chest motions resulting from a subject's heartbeat. The 
heart rate estimates calculated from the primary peak locations of the ECG signal were verified as accurate through visual inspection of the time domain ECG signal.

\subsection{Results}

All figures in this chapter are taken from subject 3 as representative data from all subjects.

\subsubsection{Tests with Subjects Seated}

All figures in this section are taken from the third sitting test of subject 3 as representative data for all subjects during sitting tests. Figure 5.3 is a plot of the radar, RIP band, and ECG signal. Five segments are labeled in this figure. The first segment represents the section of regular breathing $(\mathrm{RgBr})$, which is clean and periodic. The second segment represents the period of physical activity (PA) wherein the signal is noisy, aperiodic, and has a fluctuating amplitude. The third segment represents the recovery breathing segment $(\mathrm{RcBr})$ when the subject returns to regular breathing following the period of physical activity. The fourth segment represents the breath hold segment $(\mathrm{BrH})$. The fifth segment represents the deep breathing segment $(\mathrm{DBr})$ when the subject returns to regular breathing following the breath hold. The times between these five segments are considered a transitory period in the data. The recovery and deep breathing segments were both chosen to be 120 seconds long. This way, half the segment shows the return to regular breathing, and the second half shows a period of regular breathing.

Figure 5.4 shows zoomed in plots (30 seconds in length) of the regular breathing, recovery breathing, deep breathing, physical activity, and breath hold segments of the radar output signal. The regular breathing segment shows 4 breathing cycles. Both the 
recovery and deep breathing segments show 5 breathing cycles. The regular, recovery, and deep breathing segments all have good repeatability in waveform shape. The physical activity segment waveform does not have a regular periodic pattern. The breath hold signal has a smaller amplitude compared to the other segments, but shows a periodic waveform for the majority of the plot window. Figure 5.5 shows the magnitude spectrums of the regular breathing, recovery breathing, deep breathing, physical activity, and breath hold segments of the radar and RIP band signals. 


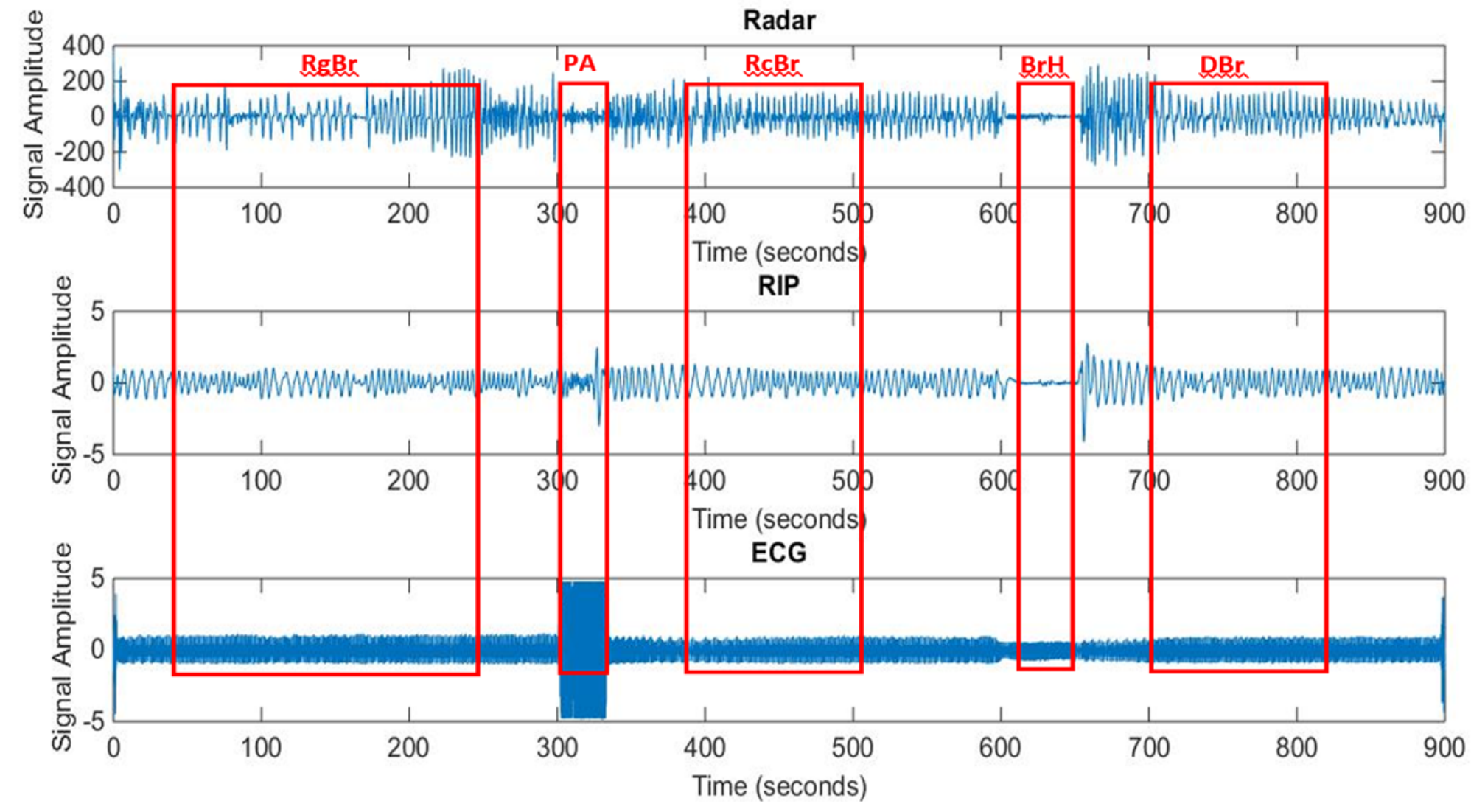

Figure 5.3 Radar output signal from the SR12003 radar unit (top), RIP band signal (middle) and ECG signal (bottom) from subject 3's third sitting test with all breathing segments $(\mathrm{RgBr}, \mathrm{PA}, \mathrm{RcBr}, \mathrm{BrH}, \mathrm{DBr})$ labelled. 


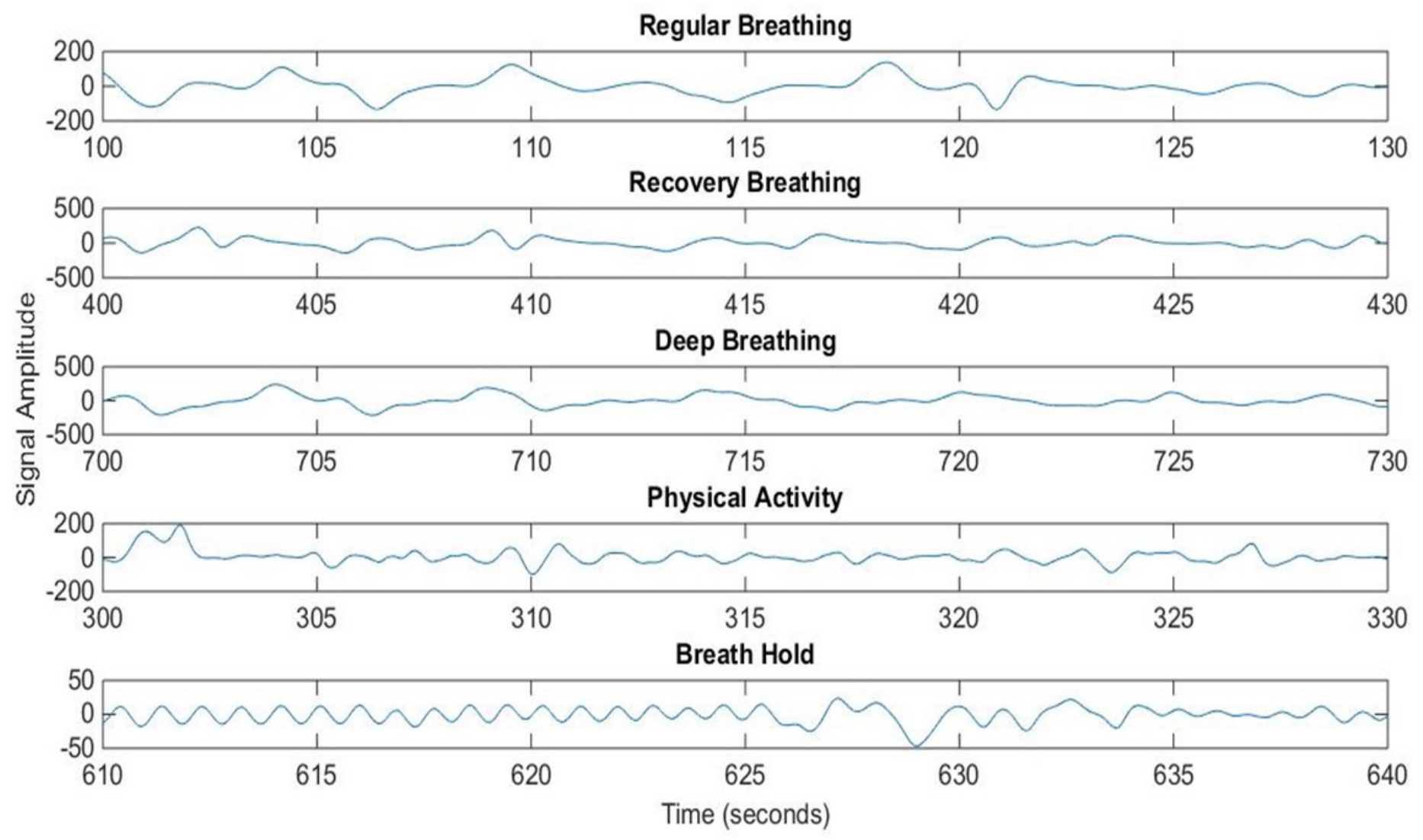

Figure 5.4 Thirty second windows of the regular breathing, recovery breathing, deep breathing, physical activity, and breath hold segments from Subject 3's third sitting test. 

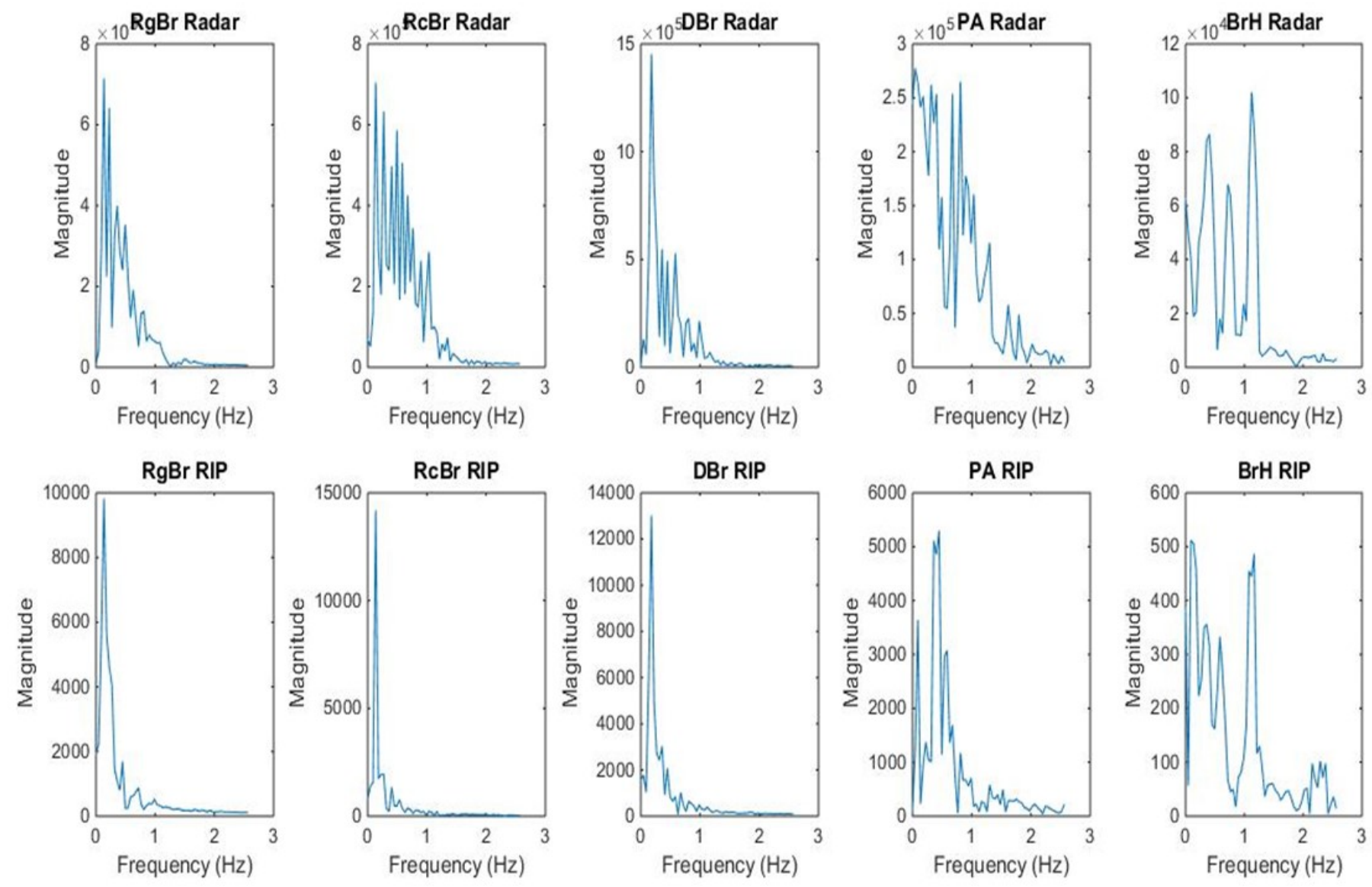

Figure 5.5 Magnitude spectrum plots for the regular breathing, recovery breathing, deep breathing, physical activity, and breath hold segments of the radar (top row) and RIP band (bottom row) signals corresponding to the analysis windows from Figure 5.4. 
Table 5.3 compares the primary peak frequencies from the radar magnitude spectrums with the breathing rate manually calculated by measuring the peak to peak distance in the RIP band time domain signals.

Table 5.3 Primary peak locations of the radar output signal obtained from the magnitude spectrums and the calculated breathing rate of the RIP band signals corresponding to the analysis windows from Figure 5.4.

\begin{tabular}{|c|c|c|}
\hline Regular Breathing Segment & Primary Peak $(\mathrm{Hz})$ & Breathing Rate (brpm) \\
\hline Radar & 0.135 & 8 \\
\hline RIP Band & 0.135 & 8 \\
\hline Recovery Breathing Segment & Primary Peak (Hz) & Breathing Rate (brpm) \\
\hline Radar & 0.135 & 8 \\
\hline RIP Band & 0.135 & 8 \\
\hline Deep Breathing Segment & Primary Peak (Hz) & Breathing Rate (brpm) \\
\hline Radar & 0.180 & 11 \\
\hline RIP Band & 0.180 & 11 \\
\hline Physical Activity Segment & Primary Peak (Hz) & Breathing Rate (brpm) \\
\hline Radar & 0.808 & 48 \\
\hline RIP Band & 0.449 & 27 \\
\hline Breath Hold Segment & Primary Peak (Hz) & Breathing Rate (brpm) \\
\hline Radar & 1.122 & 67 \\
\hline RIP Band & - & 0 \\
\hline
\end{tabular}




\subsubsection{Tests with Subject Standing}

All figures in this section are taken from the third standing test of subject 3 as representative data for all subjects during standing tests.

Figure 5.6 is a plot of the radar, RIP band, and ECG signal. The same five segments (regular breathing, recovery breathing, deep breathing, physical activity, and breath hold) were analysed as in section 5.4.1.

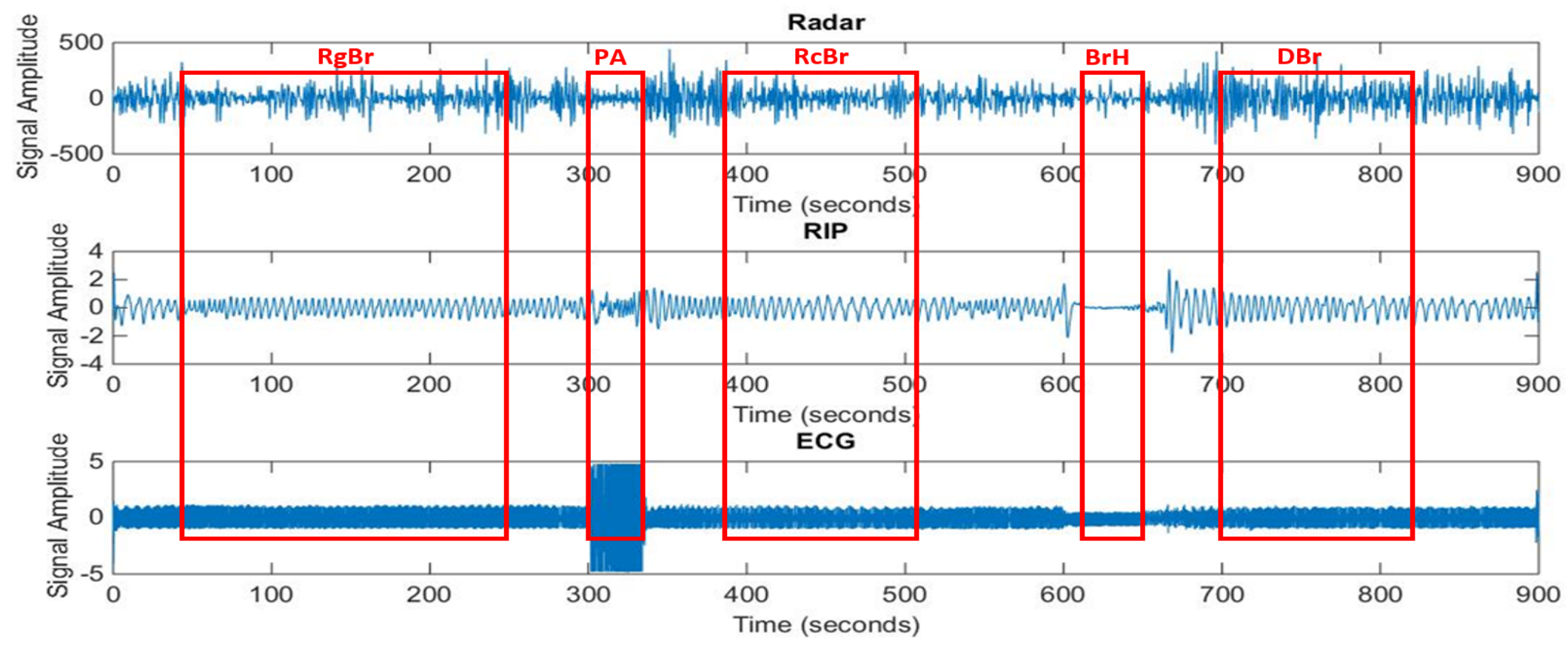

Figure 5.6 Radar output signal from the SR12003 radar unit (top), RIP band signal (middle), and ECG signal (bottom) from subject 3's third standing test. 
Figure 5.7 shows zoomed in plots (30 seconds in length) of the regular breathing, recovery breathing, deep breathing, physical activity, and breath hold segments of the radar output signal. The regular, recovery, and deep breathing segments are much noisier than in the sitting tests, and the signal waveforms are not as repeatable making it more difficult to estimate the number of breath cycles in the window using visual inspection. The regular breathing pattern appears to show 6 breath cycles. The recovery breathing segment appears to show 5 breath cycles. The deep breathing segment appears to show 5 or 6 breath cycles. The physical activity signal waveform has poor repeatability, and the breath hold shows a periodic waveform, apart from the middle part of the analysis window.

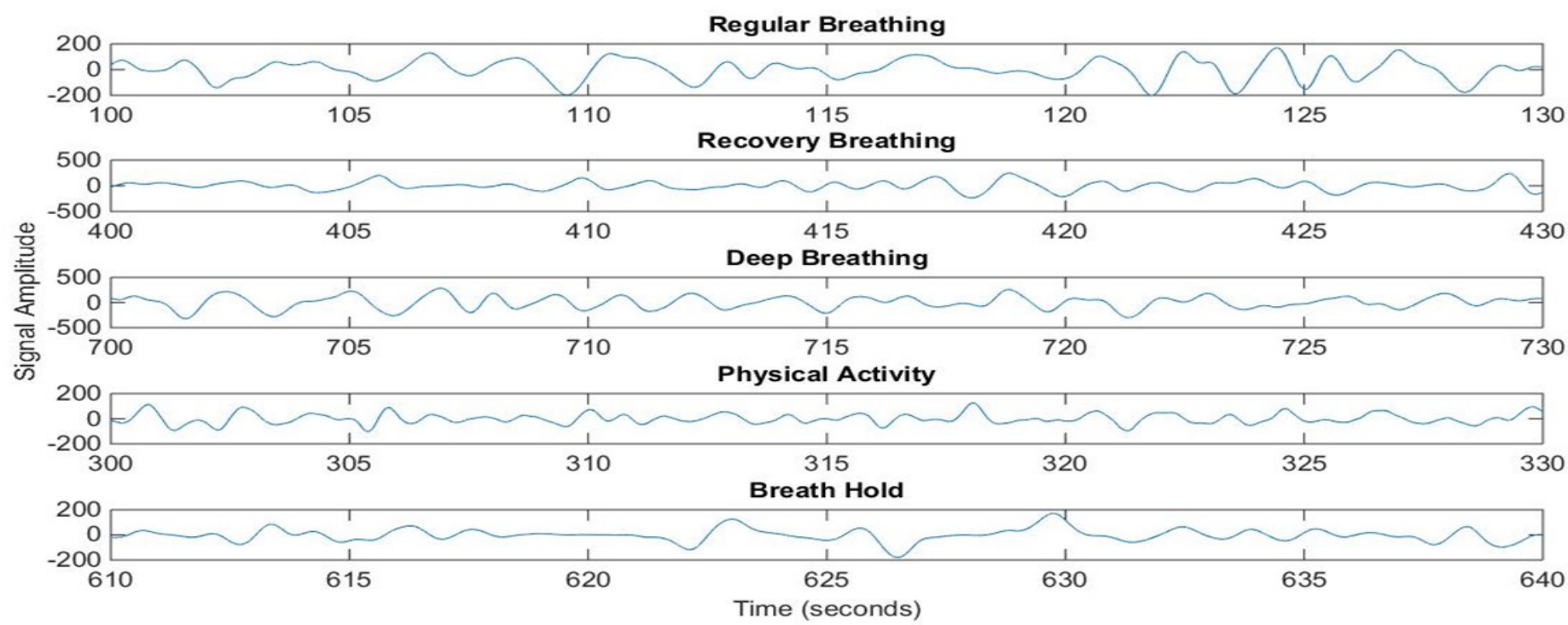

Figure 5.7 Thirty second windows of the regular breathing, recovery breathing, deep breathing, physical activity, and breath hold segments from Subject 3's third standing test. 
Figure 5.8 shows the magnitude spectrums of the regular breathing, recovery breathing, deep breathing, physical activity, and breath hold segments of the radar and RIP band signals.
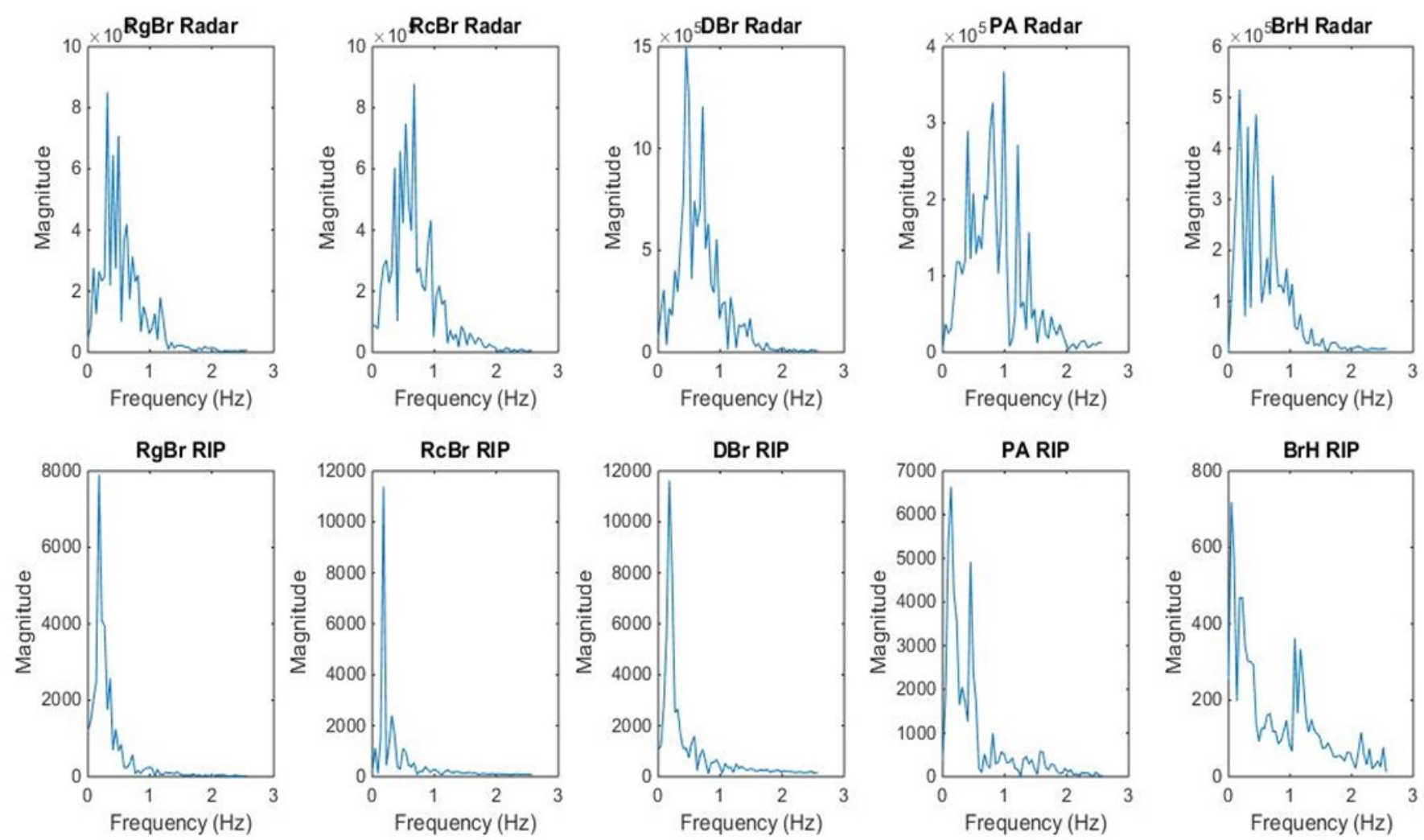

Figure 5.8 Magnitude spectrum plots for the regular breathing, recovery breathing, deep breathing, physical activity, and breath hold segments of the radar (top row) and RIP band (bottom row) signals corresponding to the analysis windows from Figure 5.7. 
Table 5.4 compares the primary peak frequencies with of the radar segments with the breathing rate calculated by measuring the peak to peak distance in the RIP band time domain signals.

Table 5.4 Primary peak locations of the radar output signal obtained from the magnitude spectrums and the calculated breathing rate of the RIP band signals corresponding to the analysis windows from Figure 5.7.

\begin{tabular}{|c|c|c|}
\hline Regular Breathing Segment & Primary Peak (Hz) & Breathing Rate (brpm) \\
\hline Radar & 0.314 & 19 \\
\hline RIP Band & .177 & 11 \\
\hline $\begin{array}{c}\text { Recovery Breathing } \\
\text { Segment }\end{array}$ & Primary Peak (Hz) & Breathing Rate (brpm) \\
\hline Radar & 0.359 & 22 \\
\hline RIP Band & 0.180 & 11 \\
\hline Deep Breathing Segment & Primary Peak (Hz) & Breathing Rate (brpm) \\
\hline Radar & 0.404 & 24 \\
\hline RIP Band & 0.180 & 11 \\
\hline Physical Activity Segment & Primary Peak (Hz) & Breathing Rate (brpm) \\
\hline Radar & 0.988 & 59 \\
\hline RIP Band & 0.449 & 27 \\
\hline Breath Hold Segment & Primary Peak (Hz) & Breathing Rate (brpm) \\
\hline Radar & .180 & 11 \\
\hline RIP Band & - & 0 \\
\hline
\end{tabular}

\subsubsection{Breathing Estimation Algorithm}

Figure 5.9 shows box plots of the error in the breathing rate estimate for each signal segment from all three of subject 3's sitting tests. This error is calculated by computing the difference between the breathing rates associated with the peak frequencies in the radar output signal and RIP band signal spectrums. 


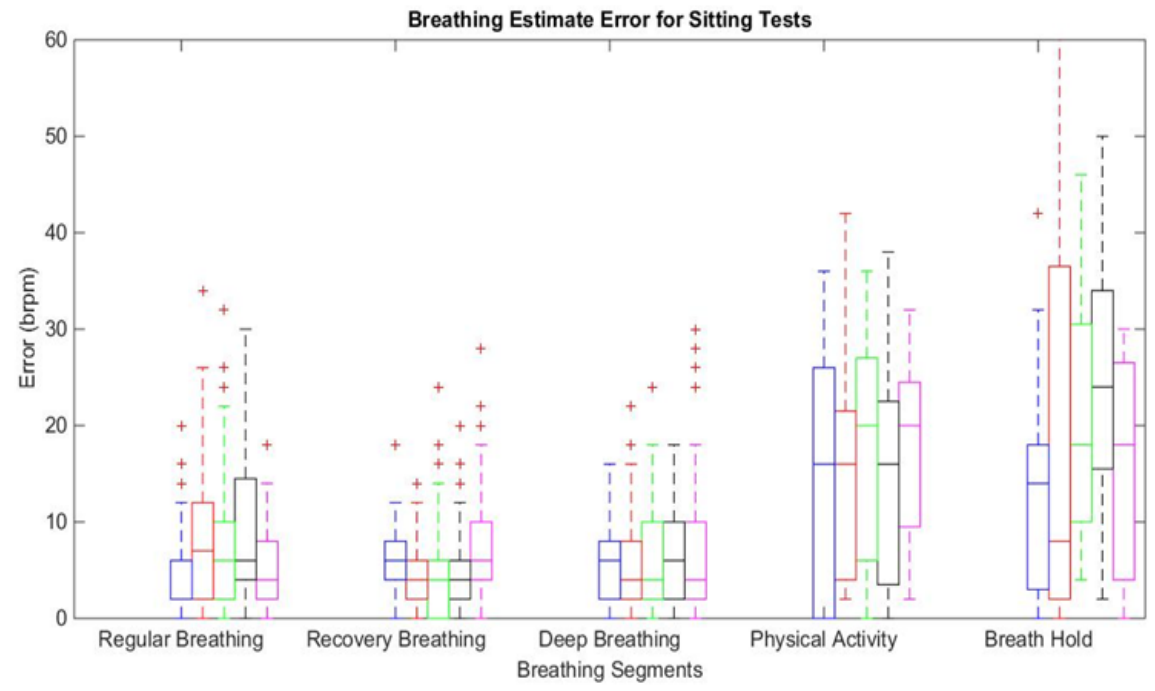

Figure 5.9 Box plots of the breathing estimate error for all five breathing segments from the sitting tests of each subject (subject 1 - blue, subject 2 - red, subject 3 - green, subject 4 - black, subject 5 magenta).

Figure 5.10 shows box plots of the error in the breathing rate estimate for each signal segment from all three of subject 3's standing tests.

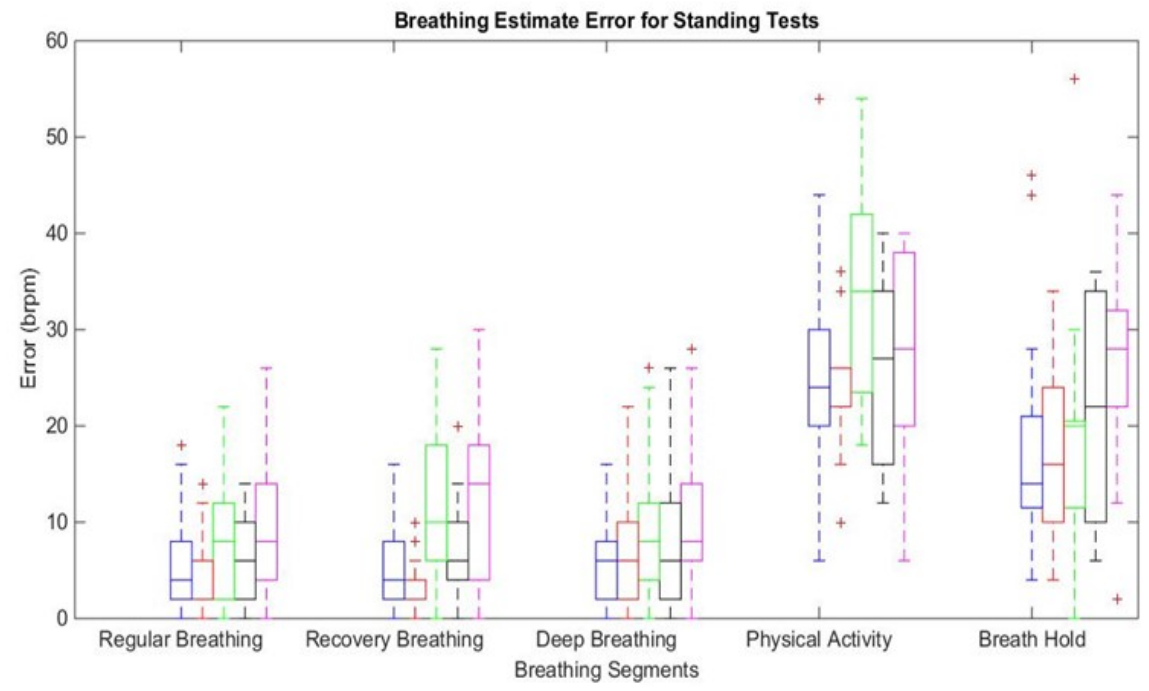

Figure 5.10 Box plots of the breathing estimate error for all five breathing segments from the standing tests of each subject (subject 1 - blue, subject 2 - red, subject 3 - green, subject 4 - black, subject 5 - magenta).

Table 5.5 shows the mean and standard deviation values of breathing estimate error for each breathing segment from each subject's sitting tests. 
Table 5.5 Mean and standard deviation of breathing estimate error in each breathing segment for all sitting tests from each subject.

\begin{tabular}{|c|c|c|c|c|c|}
\hline \multirow{2}{*}{ Subject (\#) } & \multicolumn{5}{|c|}{ Mean and Standard Deviation of Error (brpm) } \\
\cline { 2 - 6 } & $\mathbf{R g B r}$ & $\mathbf{R c B r}$ & $\mathbf{D B r}$ & $\mathbf{P A}$ & BrH \\
\hline $\mathbf{1}$ & $5 \pm 5$ & $6 \pm 4$ & $5 \pm 4$ & $14 \pm 13$ & $13 \pm 11$ \\
\hline $\mathbf{2}$ & $5 \pm 4$ & $5 \pm 3$ & $6 \pm 5$ & $15 \pm 12$ & $20 \pm 25$ \\
\hline $\mathbf{3}$ & $7 \pm 6$ & $5 \pm 5$ & $6 \pm 5$ & $18 \pm 11$ & $21 \pm 12$ \\
\hline $\mathbf{4}$ & $9 \pm 8$ & $5 \pm 4$ & $7 \pm 5$ & $16 \pm 13$ & $26 \pm 16$ \\
\hline $\mathbf{5}$ & $5 \pm 4$ & $8 \pm 7$ & $7 \pm 7$ & $17 \pm 9$ & $16 \pm 11$ \\
\hline Average & $\mathbf{6} \pm \mathbf{6}$ & $\mathbf{6} \pm \mathbf{5}$ & $\mathbf{6} \pm \mathbf{5}$ & $\mathbf{1 6} \pm \mathbf{1 2}$ & $\mathbf{1 9} \pm \mathbf{1 6}$ \\
\hline $\mathbf{5}$ (SR2003) & $6 \pm 6$ & $10 \pm 6$ & $7 \pm 7$ & $12 \pm 12$ & $14 \pm 6$ \\
\hline
\end{tabular}

Table 5.6 shows the mean and standard deviation values of breathing estimate error for each breathing segment from each subject's standing tests.

Table 5.6 Mean and standard deviation of breathing estimate error in each breathing segment for all standing tests from each subject.

\begin{tabular}{|c|c|c|c|c|c|}
\hline \multirow{2}{*}{ Subject (\#) } & \multicolumn{5}{|c|}{ Mean and Standard Deviation of Error (brpm) } \\
\cline { 2 - 6 } & RgBr & RcBr & DBr & PA & BrH \\
\hline $\mathbf{1}$ & $5 \pm 4$ & $6 \pm 4$ & $6 \pm 4$ & $26 \pm 12$ & $18 \pm 11$ \\
\hline $\mathbf{2}$ & $4 \pm 3$ & $4 \pm 2$ & $7 \pm 6$ & $25 \pm 7$ & $17 \pm 10$ \\
\hline $\mathbf{3}$ & $8 \pm 6$ & $9 \pm 8$ & $9 \pm 7$ & $33 \pm 10$ & $18 \pm 12$ \\
\hline $\mathbf{4}$ & $6 \pm 4$ & $7 \pm 4$ & $7 \pm 5$ & $26 \pm 9$ & $22 \pm 11$ \\
\hline $\mathbf{5}$ & $9 \pm 6$ & $12 \pm 8$ & $10 \pm 6$ & $28 \pm 9$ & $26 \pm 10$ \\
\hline Average & $\mathbf{6} \pm \mathbf{5}$ & $\mathbf{8} \pm \mathbf{6}$ & $\mathbf{8} \pm \mathbf{6}$ & $\mathbf{2 8} \pm \mathbf{1 0}$ & $\mathbf{2 0} \pm \mathbf{1 1}$ \\
\hline
\end{tabular}

\subsection{Discussion}

By visually inspecting the time domain, it can be seen that the radar output signal provided a clearer breathing signal when the subject was seated. The radar output signal is much cleaner when the subject is sitting compared to standing. This is not surprising since the subject is less likely to move when they can comfortably sit back in a chair. When a subject is standing, they will sway back and forth despite their best efforts to remain as still as possible. When the radar was positioned vertically compared to horizontally, the quality of the radar output signal did not appear to improve. 
Additionally, the one test conducted using the SR2003 radar unit did not provide any noticeable improvements in signal quality. Figure 5.11 shows the same analysis window from the regular breathing segment of three different tests. The top analysis window, from subject 3's third sitting test with the radar in its horizontal orientation, shows a signal waveform with good repeatability. The middle analysis window is from subject 5's second sitting test with the radar in its vertical orientation, and the bottom analysis window is from subject 5's fourth sitting test with the SR2003 radar. When the radar is oriented vertically, the signal waveform is not repeatable and appears to be noisier. With the SR2003 radar unit, the signal waveform does have good repeatability but is not as clear as the signal from subject 3's third sitting test. The noisier signals could be due to the fact that the tests with subject 5 were conducted in a room with more sources of reflection, as opposed to previous tests which were conducted in the Vicon room.
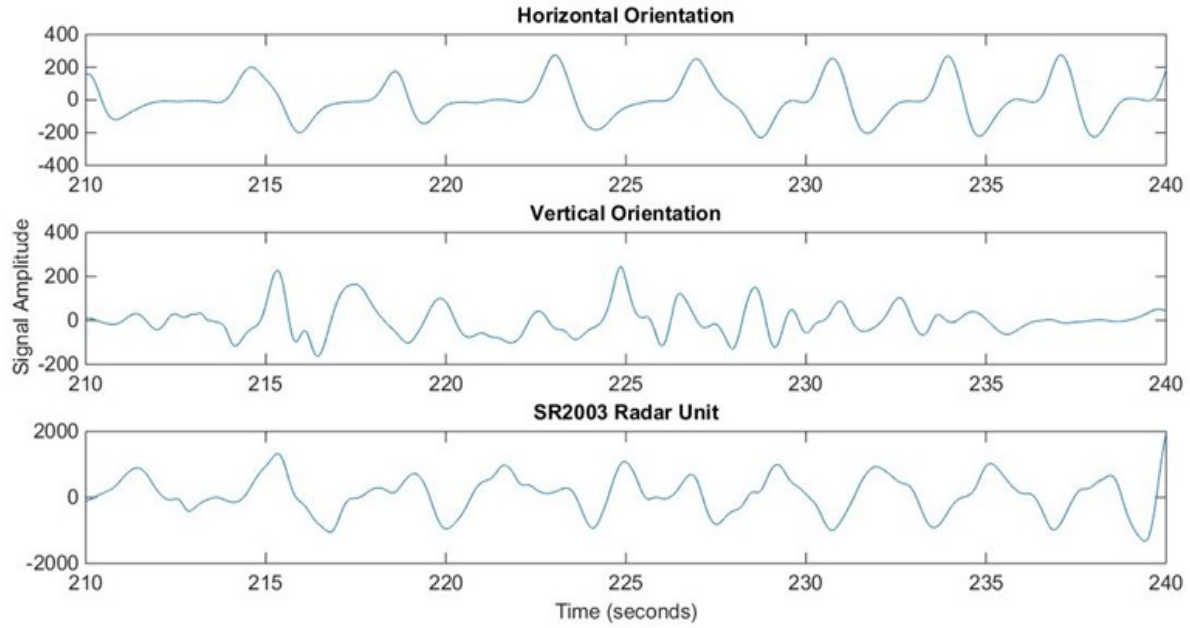

Figure 5.11 Plot of regular breathing segments from test with radar in horizontal orientation (top), test with radar in vertical orientation (middle), and from test using the SR2003 radar unit.

The same conclusions can be drawn from the frequency domain analysis of the different test conditions. In the regular breathing, recovery breathing, and deep breathing segments, the radar output signal spectrum has a peak associated with a breathing rate 
close to the rate manually calculated from the RIP band signal. For sitting tests, the mean error between the peak rate and the manually calculated rate is on average $0.1 \mathrm{~Hz}$ or 6 brpm; for standing tests, the difference is slightly higher, between $0.1 \mathrm{~Hz}-0.133 \mathrm{~Hz}$ or 6 brpm -8 brpm (see Tables 5.5 and 5.6). This is promising because it provides evidence that the radar can estimate breathing rate. Ideally, we would expect mean error values closer to the estimate resolution of 2 breaths per minute; however, the radar system is a contactless monitoring method, estimating the breathing rate based on the detected motion, observed at a distance. The radar monitoring system is much more susceptible to noise, interference, and artifacts and is not be expected to be as accurate at estimating breathing rate as the RIP band. Changing the orientation of the radar (from horizontal to vertical) resulted in a higher mean error in both sitting and standing positions. The mean error from the SR2003 test was the same as the average error (from all 5 subjects) for the regular breathing segment, but slightly higher than the average error for the recovery and deep breathing segments.

Many of the large errors which occur for both the sitting and standing tests seem to be a result of harmonic peaks, which have higher amplitudes than the peak corresponding to the breathing rate. Other potential sources of noise may include motion due to swaying, and small involuntary movements by the subject. Figure 5.12 shows two cases (from the regular and recovery breathing segments) where the peak of a harmonic has a higher amplitude, and as a result of this, would be considered the primary peak and associated with the estimated breathing rate. 



Figure 5.12 Radar and RIP band magnitude spectrums showing the presence of harmonic peaks with larger amplitudes than the peak which should be associated with the breathing rate.

In both segments, the magnitude spectrum from the radar output signal has the same peak as in the RIP band signal magnitude spectrum, but the harmonic peaks present in the radar magnitude spectrums have higher amplitudes. The algorithm looks for the primary peaks (the harmonic peaks in these cases) and associates them with the estimated breathing rate of the subject. The breathing rates of the harmonic peaks are approximately twice the actual breathing rate of the subject (see Table 5.4), resulting in large estimate error. These cases where the harmonic peaks with large amplitudes occur suggest that finding the primary peak is not the optimal solution of the algorithm. A better estimate could be achieved via harmonic analysis [46]; this should be explored in future work.

For the physical activity segments, similar spectrums occur for both sitting and standing tests in that the rate estimates associated with the locations of the primary peaks in the radar spectrum do not match up with the manually calculated rate. The radar output signal spectrums all have multiple peaks of similar magnitude, making it difficult to estimate a breathing rate. This is not unexpected because the large body motions would 
obscure the breathing motion in the radar output signal; breathing estimates would not be reliable during these segments.

For the breath hold segments, the breathing rate should be zero; however, the breathing algorithm will always find a peak in the radar output signal spectrum, resulting in an incorrect breathing rate.

Frequency domain analysis of the radar, RIP band, and ECG signal shows a promising result. Figure 5.13 shows the magnitude spectrum of the radar, RIP band, and ECG signal from subject 3's third sitting test.
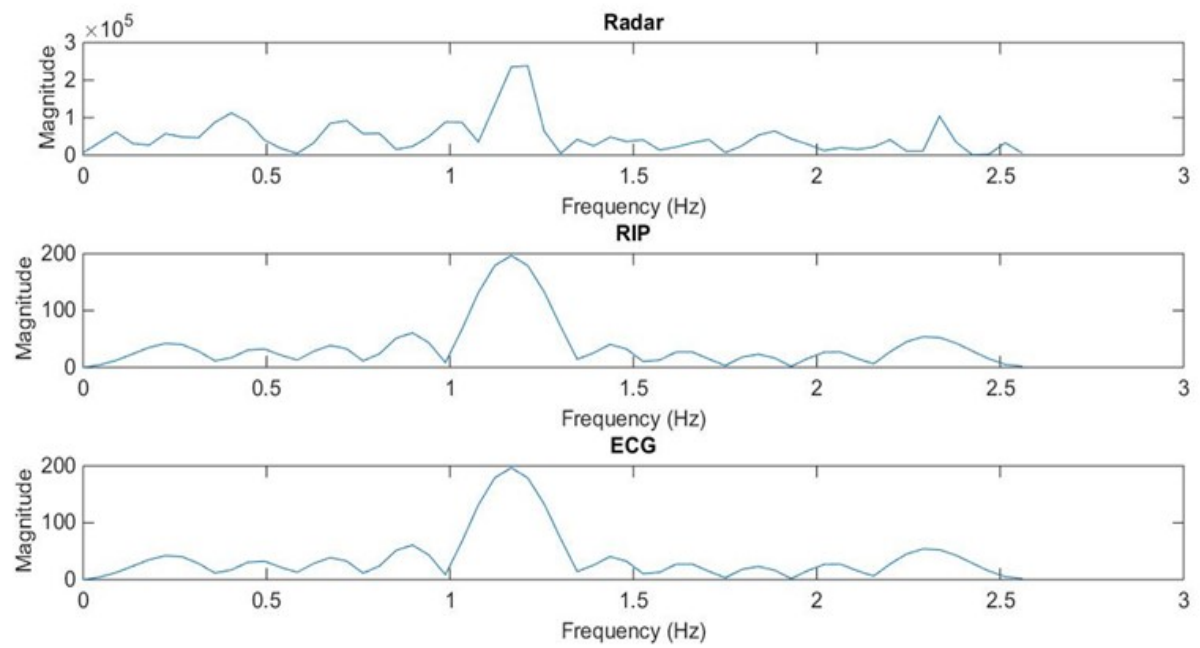

Figure 5.13 Magnitude spectrum of Radar (top), RIP band (middle), and ECG (bottom) signal during breath hold spectrum from subject 3 's third sitting test.

All three signal spectrums share a common primary peak, which is evidence of the radar's ability to discern and estimate a person's heart rate. Table 5.7 shows the mean and standard deviation values of heart rate error for the breath hold segment from each subject's sitting and standing tests, as well as the mean heart rate (measured by the radar and ECG) over the breath hold segment for each subject. The average error in the heart rate for sitting tests is $5 \mathrm{bpm}$, and $6 \mathrm{bpm}$ for standing tests. The standard deviation in the 
error is the same for both sitting and standing tests. The SR2003 gave a mean error of 6 bpm; $1 \mathrm{bpm}$ higher than the average error for sitting tests from all subjects.

Table 5.7 Mean and standard deviation of heart rate estimate error from breath hold segment for all sitting and standing tests from each subject, and average heart rate over the breath hold for each subject.

\begin{tabular}{|c|c|c|c|c|}
\hline \multirow{2}{*}{ Subject \# } & \multicolumn{2}{|c|}{$\begin{array}{c}\text { Mean and Standard Deviation of } \\
\text { Error } \mathbf{( b p m )}\end{array}$} & \multicolumn{2}{c|}{$\begin{array}{c}\text { Average Heart Rate } \\
\text { (bpm) }\end{array}$} \\
\cline { 2 - 5 } & $\begin{array}{c}\text { Breath Hold } \\
\text { while Sitting }\end{array}$ & $\begin{array}{c}\text { Breath Hold } \\
\text { while Standing }\end{array}$ & Radar & ECG \\
\hline $\mathbf{1}$ & $5 \pm 5$ & $6 \pm 4$ & 61 & 65 \\
\hline $\mathbf{2}$ & $1 \pm 3$ & $5 \pm 4$ & 70 & 72 \\
\hline $\mathbf{3}$ & $6 \pm 3$ & $6 \pm 6$ & 61 & 66 \\
\hline $\mathbf{4}$ & $5 \pm 4$ & $7 \pm 4$ & 58 & 63 \\
\hline $\mathbf{5}$ & $6 \pm 6$ & $7 \pm 4$ & 66 & 73 \\
\hline Average & $\mathbf{5} \pm \mathbf{4}$ & $\mathbf{6} \pm \mathbf{4}$ & $\mathbf{6 3}$ & $\mathbf{6 8}$ \\
\hline $\mathbf{5}$ (SR2003) & $6 \pm \mathbf{5}$ & - & 67 & 72 \\
\hline
\end{tabular}

The box plots analysing the accuracy and precision of the breathing estimation algorithm (paired with the information from Tables 5.5 and 5.6) reinforce the observations and results from the time and frequency domain analysis. It can be clearly seen that boxes for the regular, recovery, and deep breathing segments are typically smaller than the boxes for the physical activity and breath hold segments (for standing tests, the boxes are bigger because of the subject swaying). This indicates lower estimate error for the regular, recovery, and deep breathing segments, which means the algorithm can better estimate the breathing rate when the subject is stationary. The physical activity segments have a very high mean and median error which indicates that the algorithm cannot accurately estimate the breathing rate when the subject is moving around. For the breath hold segment, there is a large range of estimates and a high mean and median error. This means the algorithm cannot accurately estimate breathing rate during a breath hold but it may estimate the heart rate. 
The box plots indicate how well the algorithm can estimate breathing rates in a wide variety of scenarios/conditions; however, they do not factor in or indicate the quality of the signal when the breathing estimates are made. In the context of a vital-signs monitoring system, the signal quality must be known to decide if an estimate is correct or can be ignored.

\subsection{Conclusion}

A breathing estimation algorithm was implemented and evaluated to estimate a person's breathing rate from the radar output signal. The algorithm was created with the intent of providing real-time vital signs monitoring. Data were collected, providing a rich dataset that can be used for baseline evaluation of the breathing estimation algorithm, as it includes:

- Clean data (collected in a setting with minimal reflections and with the radar optimized such that the subject is situated right in the middle of a chosen zone)

- Data with varying breathing and heart rates (regular breathing, breath holds, and after physical activity)

- Data with varying signal quality, and

- Simultaneous ECG and RIP band data collection for gold standard measurements and comparison

Results from the testing showed that the radar could estimate a person's breathing rate, and potentially even estimate a person's heart rate in certain conditions. The breathing estimation algorithm appears to work well. When a subject was motionless, it was capable of estimating the breathing rate during periods of regular, recovery, and deep breathing. During periods of physical activity, the algorithm had trouble estimating 
breathing rate as expected. During breath holds, the algorithm incorrectly estimated the breathing rate but was capable of estimating the subject's heart rate. Although the algorithm shows promise, it should also factor in the quality of the signal to determine whether an estimate should be trusted or ignored.

Future work includes collecting further data for analysis of the algorithm and the radar. Because the tests with the SR12003 in its vertical orientation, and the SR2003, were not conducted in the Vicon room, further testing should be done to investigate their influence on the radar output signal quality. The algorithm should be modified so that it can analyse radar data in real time. Additionally, special signal filtering could be incorporated in an effort to improve the accuracy of the algorithm. 


\section{Chapter: Breathing Estimate Reliability Measure}

\subsection{Introduction}

Results shown in Chapter 5 suggest that the breathing estimation algorithm works well when a person is relatively still. When a person is sitting, the algorithm estimates the breathing rate well during periods of regular, recovery, and deep breathing, and poorly during physical activity and breath holds. When the subject is standing, the algorithm does not perform as well. An issue with the algorithm is that it does not take into account the quality of the signal, which impacts the reliability of the breathing estimate. A reliability measure is proposed in this chapter to accompany the breathing estimate. If the reliability measure is low, the breathing estimate may be ignored or discarded. If the reliability measure is high, appropriate decisions could be made using the breathing estimate. This chapter describes the methods used to quantify signal quality, which in turn is used as a measure of the reliability of the breathing estimate.

\subsection{Signal Quality Indices and Reliability Measure}

The breathing estimation algorithm analyses the radar data in the frequency domain. The following three measures of signal quality, or signal quality indices (SQIs), were investigated: 1) signal-to-noise ratio (SNR), 2) magnitude squared coherence estimate, and 3) variance. The breathing estimation algorithm uses a sliding analysis window. For each analysis window, each of the three SQI is computed to accompany the breathing estimate.

The SNR is calculated by computing the quotient of power of the signal and noise segments from the radar magnitude spectrum. Since the 30 second window provides a frequency resolution of $0.033 \mathrm{~Hz}$, the signal segment is a window centered on the largest 
peak extending $0.033 \mathrm{~Hz}$ on either side of the peak; the signal segment is comprised of the peak value, and one value on either side of the peak. Anything outside this window between $0 \mathrm{~Hz}$ and $2 \mathrm{~Hz}$ is considered noise for the purposes of the SNR calculation. The signal power is the sum of the absolute squares of its time-domain samples divided by the signal length, or equivalently, the square of its RMS level; the noise power is the square of its RMS level. Figure 6.1 below shows the signal and noise segments from one time window. If the SNR is high, the signal is clean with little noise, and the breathing estimate should be accurate. If the SNR is low, the signal will be very noisy and the estimate will be unreliable.

The magnitude squared coherence function compares the magnitude spectrum of the radar output signal in the current window with the magnitude spectrum of the radar output signal in the previous window. It provides an estimate which is a function of frequency with values between 0 and 1 that indicates how well the current window's radar spectrum corresponds to the previous window's radar spectrum at each frequency [58]. It is a function (see equation 6.1) of the power spectral densities, $P_{x x}(f)$ and $P_{y y}(f)$, of the current analysis window (x) and the previous analysis window (y), and the cross power spectral density, $P_{x y}(f)$, of x and y:

$$
C_{x y}(f)=\frac{\left|P_{x y}(f)\right|^{2}}{P_{x x}(f) P_{y y}(f)}[58]
$$

If the magnitude squared coherence is high (close to 1), then it means that the locations of peaks in the magnitude spectrums of the current and previous window match up. If it is low (close to 0 ), then the magnitude spectrums of the current and previous analysis window differ. 
Each analysis window looks at the magnitude of the radar output signal amplitude $R$, composed of $N$ samples. Variance, $V$, is computed as follows:

$$
V=\frac{1}{N} \sum_{i=1}^{N}\left|R_{i}-\mu\right|^{2}[59]
$$

Where $\mu$ is the mean of the radar output signal amplitude. Variance can tell us if the signal is noisy, or if there is a low energy signal. If a signal is noisy, the variance should be high because the amplitude will be fluctuating around the mean. If there is a low energy signal, there will be almost zero variance because the signal amplitude will be very low; this would be useful in such cases as breathing cessation.

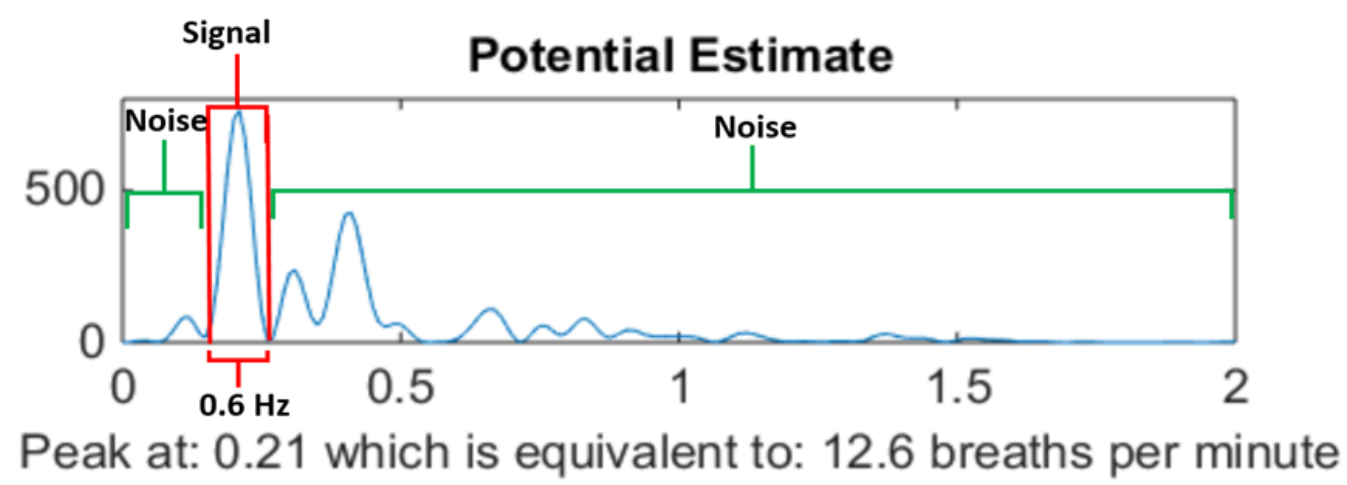

Figure 6.1 Single time window which highlights the signal and noise segments used to calculate the Signal-to-Noise SQI.

\subsection{Methodology}

The same data that were used in Chapter 5 (data from sitting and standing tests from subject 3) are used in this chapter. The breathing estimation algorithm used in this chapter is the same as was described in Chapter 5. The algorithm uses a 30 second analysis window, and the windows overlap by 5 seconds. For each analysis window, the three SQI were computed in each of the 5 breathing segments (regular breathing, recovery breathing, deep breathing, physical activity, and breath hold). SQI values from standing tests were plotted separately from the SQI values from the sitting tests. 


\subsection{Results}

Figures 6.2 and 6.3 contain box plots of the values of the SNR SQI in each of the breathing segments for the sitting tests and standing tests respectively.

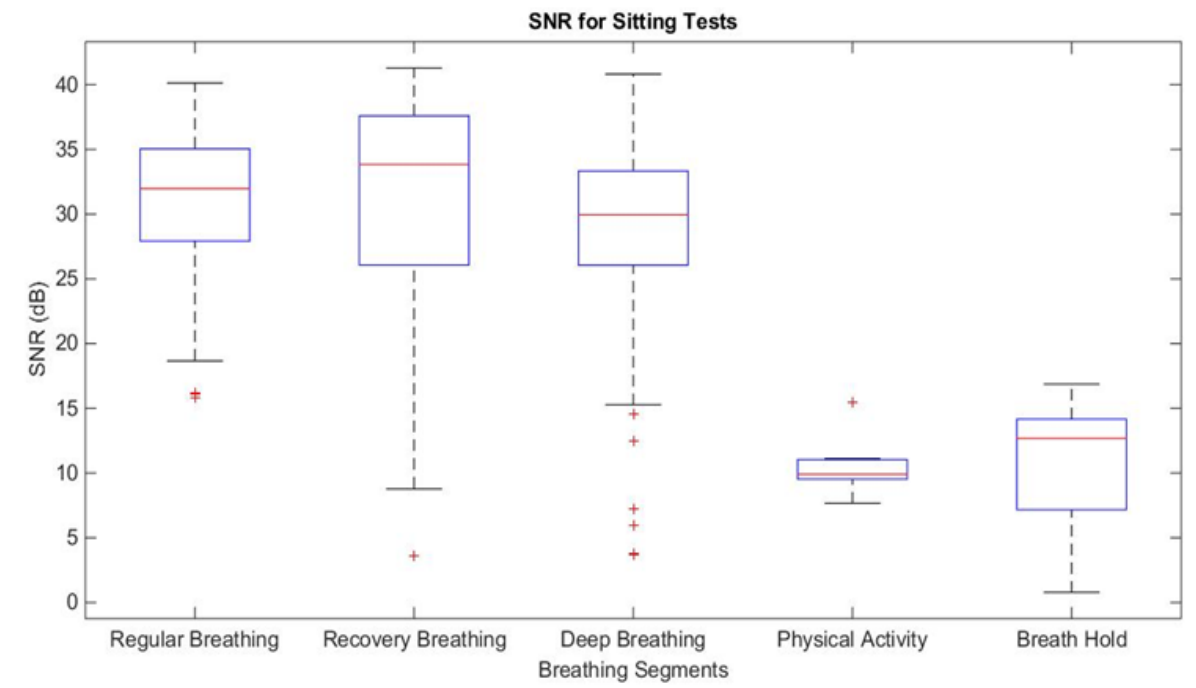

Figure 6.2 Box plot of SNR values in each breathing segment from sitting tests.

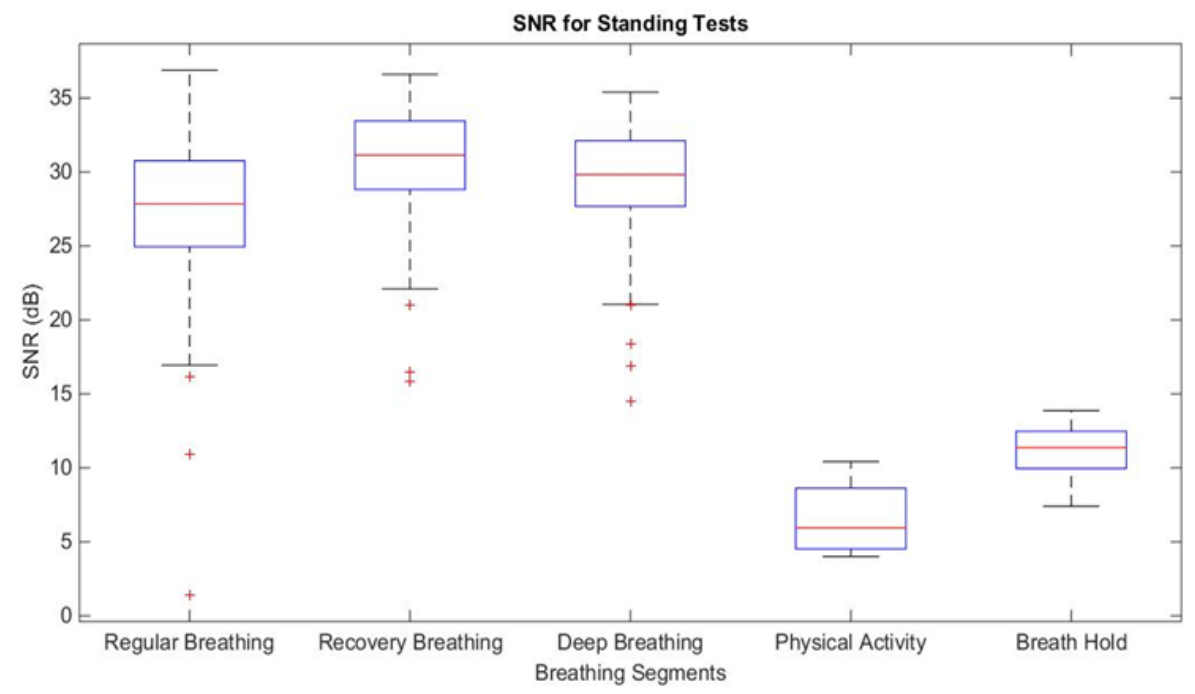

Figure 6.3 Box plot of SNR values in each breathing segment from standing tests. 
Figures 6.4 and 6.5 contain box plots of the values of the magnitude squared coherence SQI in each of the breathing segments for the sitting tests and standing tests respectively.

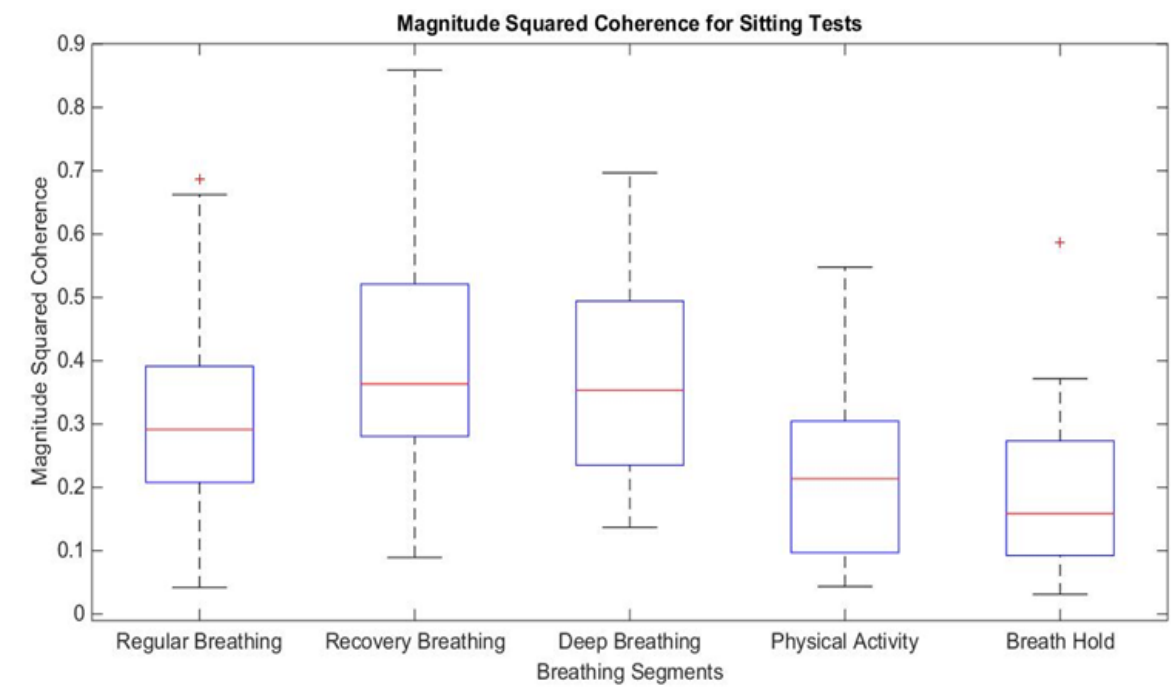

Figure 6.4 Box plot of coherence values in each breathing segment from sitting tests.

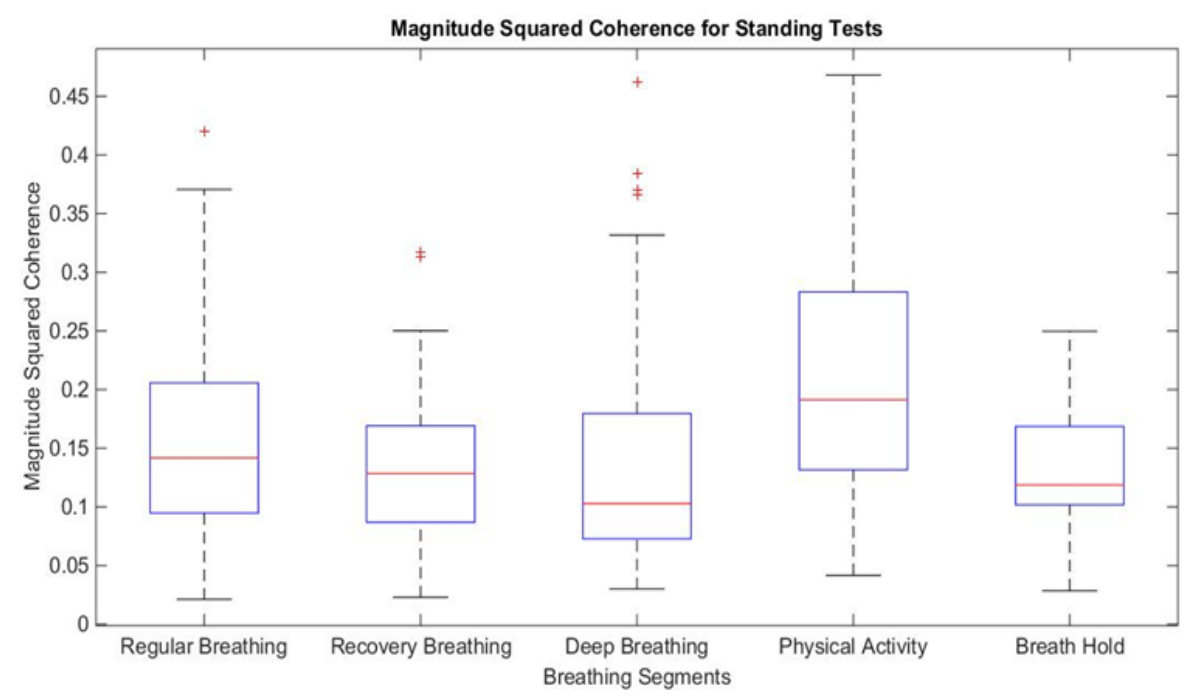

Figure 6.5 Box plot of coherence values in each breathing segment from standing tests. 
Figures 6.6 and 6.7 contain box plots of the values of the variance SQI in each of the breathing segments for the sitting tests and standing tests respectively.

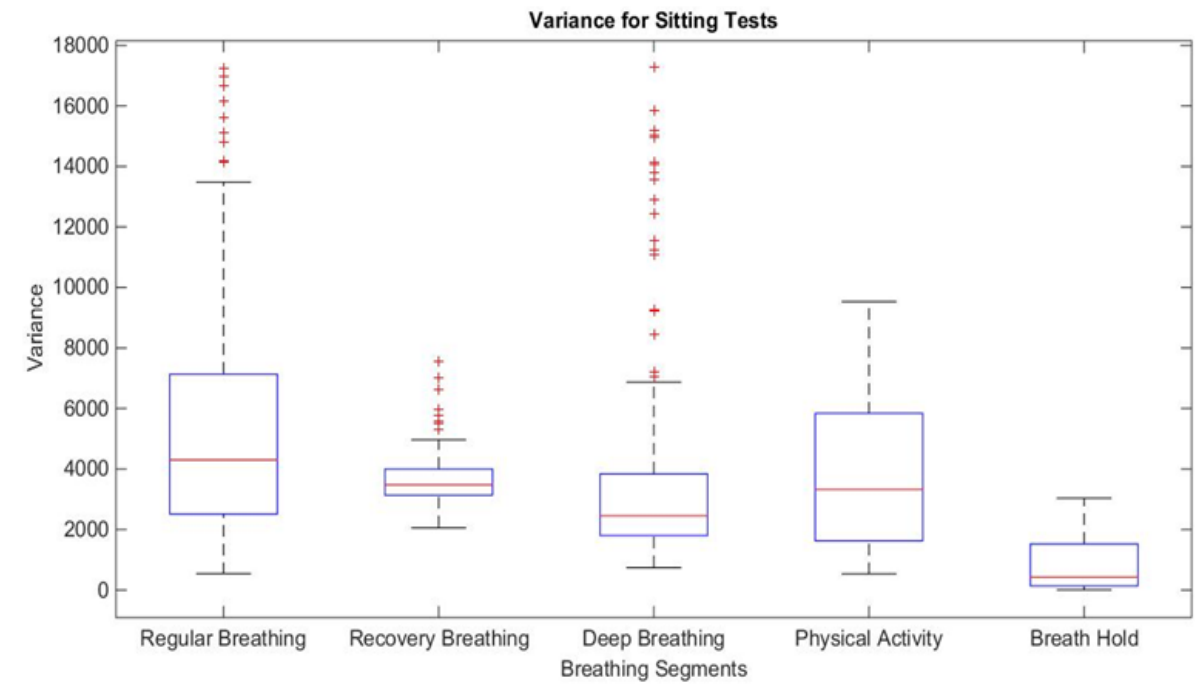

Figure 6.6 Box plot of variance values in each breathing segment from sitting tests.

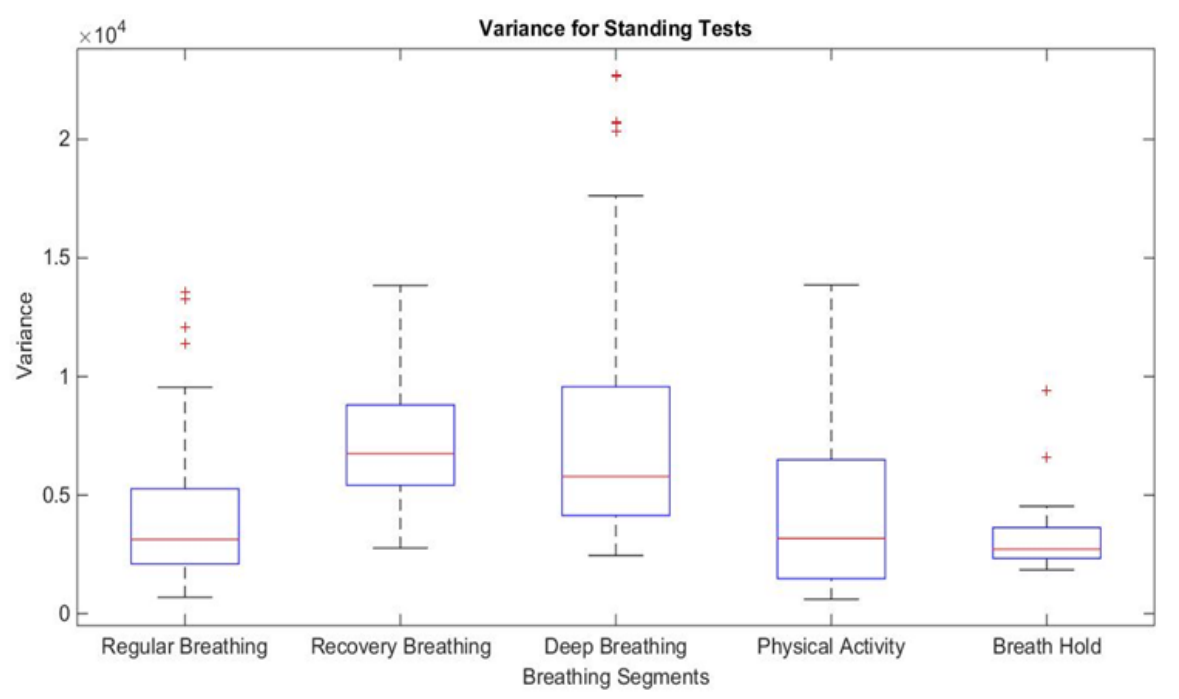

Figure 6.7 Box plot of variance values in each breathing segment from standing tests.

\subsection{Discussion}

SNR appears to work well as a reliability measure. During periods of regular, recovery, and deep breathing, when the subject is not moving, the SNR is high, providing high reliability in the breathing rate estimate, which was found to be similar to the actual 
breathing rate calculated from the RIP band (see Tables 5.5. and 5.6). During physical activity, when the subject is moving, and during the breath hold, the SNR is much lower, providing low reliability. For the physical activity segment, the SNR is lower because there is an increase in the noise power due to the additional subject motion. The SNR is lower in the breath hold segment because there is no prominent peak; the signal power goes down, while the noise power remains the same. Comparing between sitting and standing tests, the SNR values are slightly higher in the sitting tests for each segment; the mean error in the breathing rate estimate was slightly lower for sitting tests compared to standing tests (see Tables 5.5 and 5.6).

Magnitude squared coherence had mixed results as a reliability measure. For the sitting tests, the box plots show that the magnitude squared coherence is higher for the regular, recovery, and deep breathing segments, and lower for the physical activity and breath hold segments. This is a good sign but there is little difference in magnitude between the higher and lower values. For the standing tests, the box plots do not show the same pattern. Instead of the regular, recovery, and deep breathing segments having the highest magnitude squared coherence, the physical activity segment has the highest values, and the breath hold segment has similar values to the recovery breathing segment. The breath hold is not well distinguished with magnitude squared coherence because the spectrum will not likely change from window to window.

Variance looks to be a poor measure of reliability. There doesn't appear to be any way of determining signal quality using the variance, since the mean values and ranges for different segments are all similar. It was expected that the physical activity segment would have a noticeably higher variance but the results do not show this. The variance in 
the physical activity segment seems comparable to the other segments. The only segment that shows the same relative behavior between sitting and standing tests is the breath hold segment. It was expected that the breath hold segment would have a lower variance, and a small interquartile range, which it does for the sitting tests, but less so for the standing tests, which is comparable to other segments. This is expected since the radar output signal should not fluctuate greatly when the subject is holding their breath.

Figure 6.8 shows the radar output signal, breathing rate estimates from the algorithm and the RIP band, the breathing rate estimate error, and the SNR, magnitude squared coherence, and variance SQI values throughout subject 3's third sitting test.

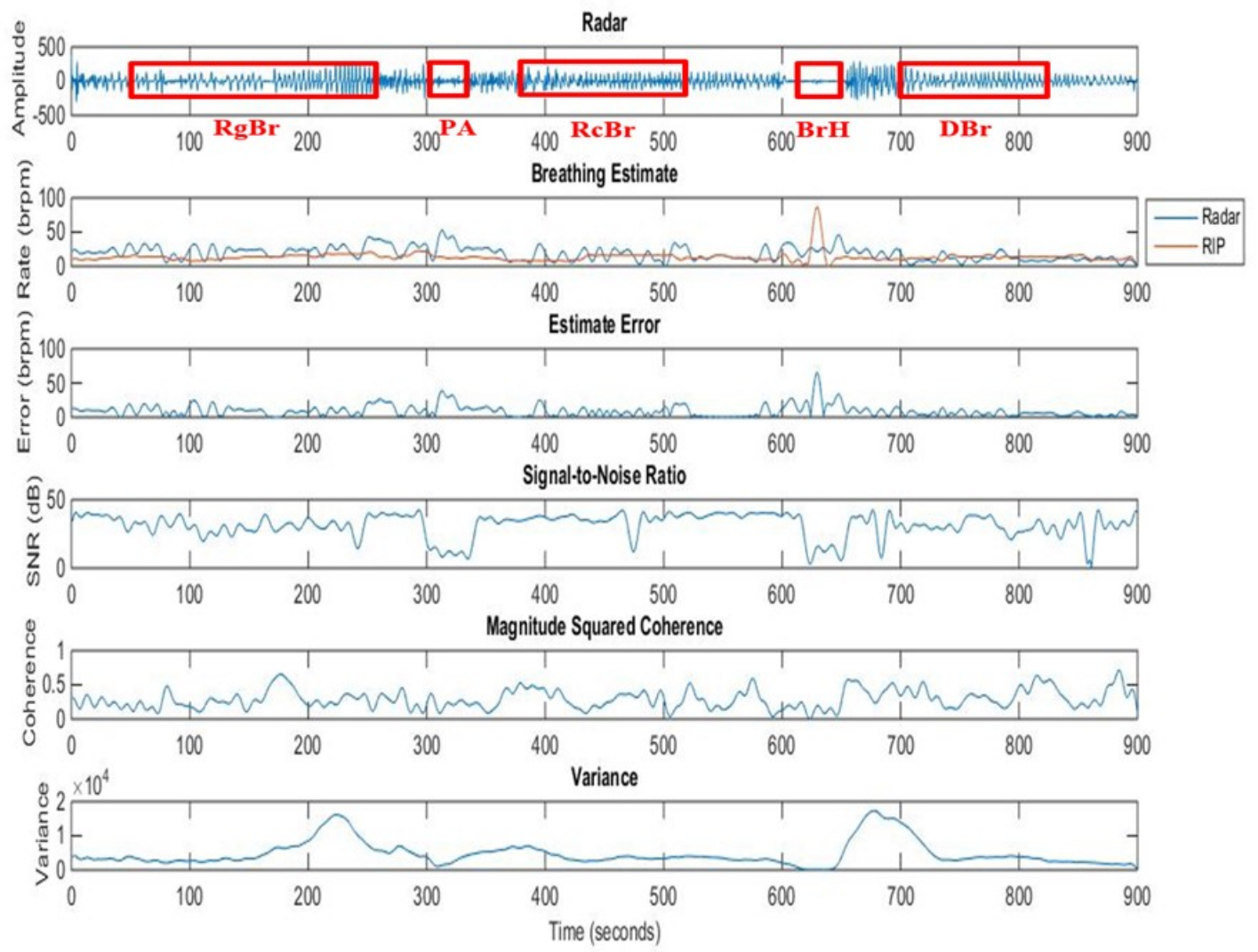

Figure 6.8 Plots of the radar output signal (top), breathing rate estimates from the algorithm and RIP band (second from top), the breathing rate estimate error (third from top), SNR values (third from bottom), coherence values (second from bottom) and variance values (b bottom) throughout subject 3's third sitting test. 
Figure 6.8 shows how the breathing rate estimates from the algorithm and RIP band, the breathing rate estimate error and SQI values change throughout the radar output signal recording. During the regular, recovery, and deep breathing segments, the two estimates are similar and the error is low. During the physical activity and breath hold segments, the estimates differ more and the error is higher. For the most part, the SNR is high during periods of normal breathing, when the breathing rate estimate error is lower, and low when the subject is moving or holding their breath, when the breathing rate estimate error is higher. The magnitude squared coherence drops slightly during the physical activity and breath hold segments and rises during the regular, recovery, and deep breathing segments, although the values fluctuate irrespective of the breathing rate estimate error. The variance also fluctuates irrespective of the breathing rate estimate error, but does decrease during the physical activity and breath hold segments, as with the magnitude squared coherence.

\subsection{Conclusion}

An analysis of signal quality indices was done to improve the performance of the breathing algorithm by taking into account the quality of the signal, improving the reliability of the breathing rate estimates. Signal-to-noise ratio, magnitude squared coherence, and variance were used to measure the quality of the radar output signal. The performance of each SQI was compared with to the others.

Of the different signal quality indices, the SNR appears to be the best at quantifying signal quality. The magnitude squared coherence does not perform as well as the SNR SQI but still shows some promise. Variance does not appear to do very well and does not seem to be a useful SQI. 
The signal-to-noise ratio signal quality index will improve the reliability of the breathing rate estimates. When a person is not moving, the breathing estimation algorithm can estimate the breathing rate, and there is a high SNR, resulting in high reliability. When a person is moving however, the algorithm gives both good and bad estimates, but since there are lower SNR values, there is low reliability in the estimates so they can be ignored.

The window size of $0.033 \mathrm{~Hz}$ was chosen empirically but was not optimized. Better performance may be gained by optimizing this value. 


\section{Chapter: Analysis of Breathing Motion}

\subsection{Introduction}

Early tests with the radar units, coupled with literature review, provided strong evidence that Doppler radar could detect a subject's breathing signal however, there is not a great deal of information on where the breathing signal originates. The research presented in this chapter performs an analysis of the breathing motion, investigating how different sections of the upper body move during breathing. This analysis helps to better understand what the radar was picking up when it returned a breathing signal. The tests described herein investigate how a subject's orientation affects the radar output signal, how much sections of the upper body contribute to the radar output signal, and provide a basic outline of movement of the upper body during breathing.

\subsection{Methodology}

\subsubsection{Effect of Orientation and Determination of Signal Source}

Tests were conducted using the SR12003 radar unit. Three tests were conducted on a 24year old male subject with no history of cardiovascular problems or disorders. The first two tests were done to investigate the effect of the subject's orientation on the radar output signal, and the last test was done to determine the source of the radar output signal.

\subsubsection{Experimental Setup}

All three tests were conducted in Carleton University's Canal Building Room 6112, which was empty, apart from a table, a few chairs (which were moved to one side of the room so as not to interfere with the testing), and a stool. The subject was seated in the stool 2 metres from the radar unit such that he was centered in Zone 5. The radar was set 
to Dwell mode and was oriented horizontally such that the beam spread vertically across the entire height of the subject. For the third test, intended to determine the source of the radar output signal, the subject had a Radio Frequency (RF) panel (which prevents any reflection of the radar output signal) to cover different sections of the body throughout the test.

\subsubsection{Experimental Procedure}

The first test was broken down into three two-minute segments with the subject changing his orientation in each segment. In the first segment, the subject was asked to face the radar. In the second segment, the subject was asked to turn sideways, and for the third segment, the subject was asked to turn such that his back was to the radar. The subject was asked to breathe normally throughout the test.

The second test was broken down into three segments as in the first test except that in the second test, the subject was asked to perform a breath hold during each segment. The subject shortly resumed normal breathing before changing his orientation and preparing to perform another breath hold.

The third test was broken down into four one-minute segments of regular breathing with the subject changing the position of the RF panel for each segment. The different positions (see Figure 7.1) are listed below:

1. The RF panel is covering everything below the subject's waist

2. The RF panel is covering the entire abdomen and lower thorax

3. The RF panel is covering much of abdomen, the entire thorax, and the neck

4. The RF panel is held up in front of the head but leaves the neck uncovered 

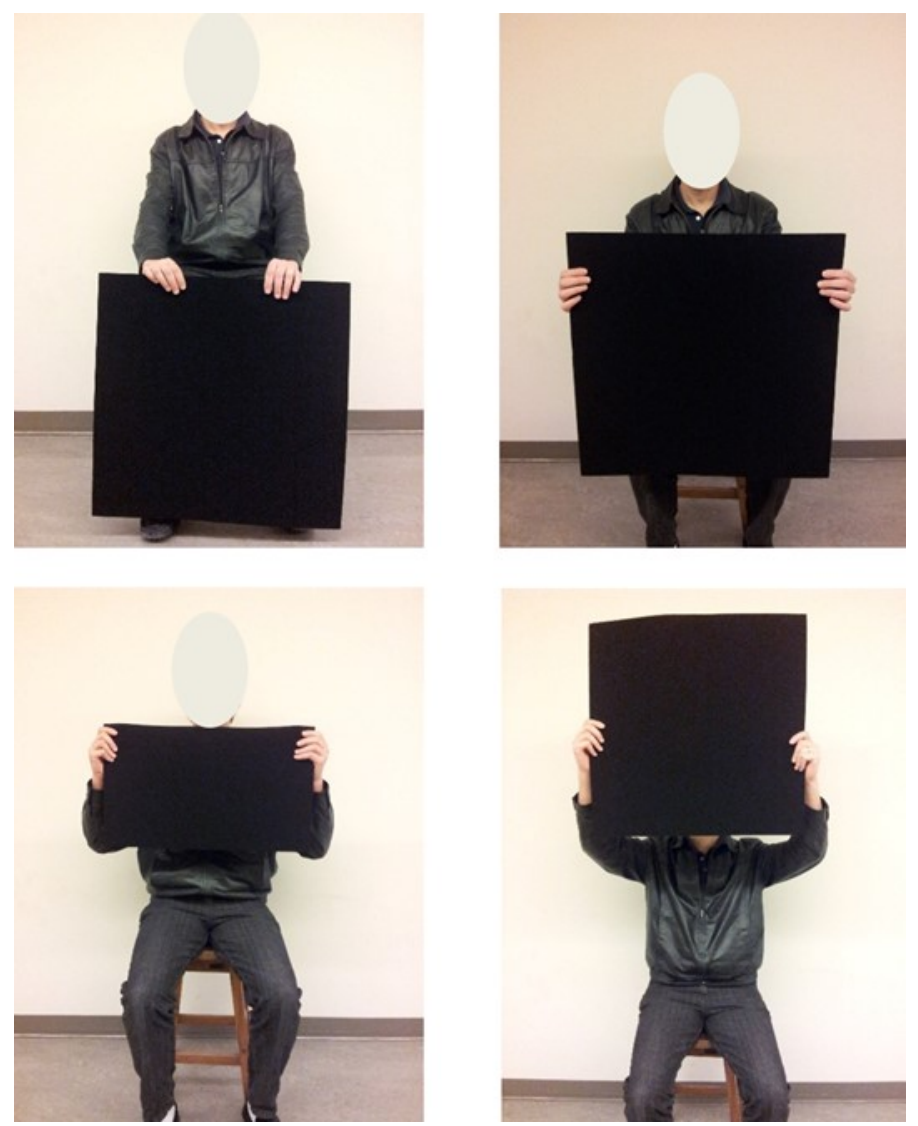

Figure 7.1 RF Panel positions. Position 1 (top left), position 2 (top right), position 3 (bottom left), and position 4 (bottom right).

\subsubsection{Analysis of Breathing Motion using Vicon System}

Tests/recordings were conducted using Carleton's Vicon motion capture system (refer to section 3.1.5 of the Methodology chapter for more information). The Vicon system was used for breathing motion analysis because of its real time tracking capabilities. The tests were conducted on three male subjects between 23 and 25 years of age with no history of cardiovascular problems or disorders. Table 7.1 below lists various physical traits such as height, weight, and waist size for each subject. 
Table 7.1 Physical traits of each subject that partook in the Vicon testing.

\begin{tabular}{|c|c|c|c|c|c|}
\hline Traits & Age & Height (cm) & Weight (kg) & $\begin{array}{c}\text { Chest Size } \\
\text { (cm) }\end{array}$ & $\begin{array}{c}\text { Waist Size } \\
\text { (cm) }\end{array}$ \\
\hline Subject 1 & 24 & 175 & 64 & 83.8 & 74.9 \\
\hline Subject 2 & 23 & 174 & 84 & 102.9 & 91.4 \\
\hline Subject 3 & 25 & 178 & 82 & 99.1 & 83.8 \\
\hline
\end{tabular}

\subsubsection{Experimental Setup}

The following is a brief description of the setup procedure and does not go into great detail; for more information, please refer to [52].

First, session files were created for all 6 recordings per subject. The cameras were then turned on and connected to the network and synced with the Vicon Blade software on the PC. The cameras' strobe intensity, threshold values, threshold grid, and sync rates were set, the cameras were calibrated using a process known as the wand wave, and the room's origin was set. The purpose of the calibration is to link the cameras to the real environment and create a relative 3-dimensional reference for each camera.

Subjects were given the choice of performing the recordings without a shirt or while wearing a dark colored (preferably black) compression shirt with no reflective surfaces.

Once chest and waist measurements were taken, reflective markers were placed on the subject. A total of 38 markers were placed on each subject. Thirty-two markers were used for the breathing motion analysis and were arranged in 4 rings of 8 markers, which were numbered as shown in Figure 7.2 and associated with one of the following four regions/sections of the upper body: 1) Lower Abdomen (purple), 2) Upper Abdomen (blue), 3) Lower Thorax (green), and 4) Upper Thorax (orange). The remaining 6 markers were used as reference markers to remove any rotation, translation, or shear movements associated with a subject swaying when sitting up without back support, or standing. Four 
of these reference markers were placed in line with the subject's hip; two on the side, one on the front, and one in the back. The remaining two reference markers were placed on the subject's shoulders. These locations of the reference markers were chosen because it was assumed that there would be negligible movement associated with breathing at these locations.


Figure 7.2 Marker Placement for Vicon testing. Red markers refer to reference markers, orange markers relate to the upper thorax region, green to the lower thorax, blue to the upper abdomen, and purple to the lower abdomen. Modified from [60].

For the recordings with the subjects seated, a stool with no back support (so as not to obscure the markers on the subject's back) was placed in the center of the room and removed following the conclusion of the recordings. When the subjects were lying down in a supine position, they were provided with a mat to lie down on, as well as pillows to put under their head and knees, for increased comfort.

\subsubsection{Experimental Procedure}

Once the reflective markers were placed on the subject, the subject was asked to stand in the center of the room, marked with tape. Each subject was instructed not to speak during 
any of the recordings, and to remain as still as possible. Six recordings were taken for each subject divided into two sets of recordings at each position. Prior to the first recording at a specific position (standing, sitting, or supine), a template recording was made where the subject was asked to breathe for 30 seconds. A template recording was made to ensure the proper labelling of markers, and to prevent temporary markers from appearing. Temporary markers are created when at least 3 cameras lose track of a marker in one frame and then reacquire the marker in a subsequent frame.

The breakdown of each recording from the first set is provided below.

1. The subject was asked to breathe normally while standing for 3 minutes.

2. The subject was asked to breathe normally while sitting for 3 minutes.

3. The subject was asked to breathe normally while in a supine position (lying down) for 3 minutes.

The second set of recordings at each position were exactly the same as the first set with the exception that prior to the start of the recording, the subjects were asked to perform jumping jacks for 30 seconds to increase their heart rate and breathing rate.

Once the final recording was completed, each recording was saved as a .c3d file. The Vicon (.c3d) files were imported into Matlab for post processing.

\subsection{Results}

\subsubsection{Effect of Orientation and Determination of Signal Source}

Figure 7.3 shows the filtered radar output signal from the first test. The orientation in each segment is labeled. One can clearly see three two-minute segments separated by large spikes in signal amplitude. The first segment, when the subject is facing the radar, has a very clear signal and the highest signal amplitude among the three segments. The 
second segment (side-facing) has the lowest signal amplitude, and the signal amplitude of the third segment (back-facing) falls in the middle.

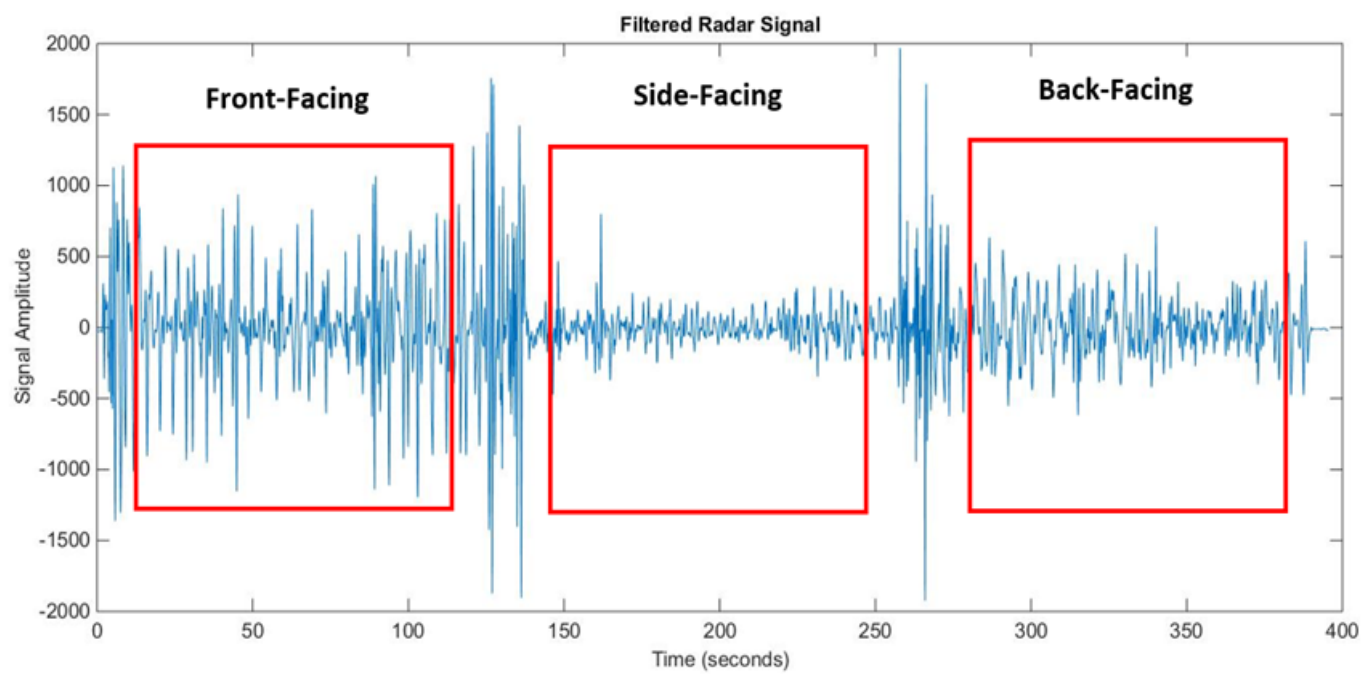

Figure 7.3 Filtered radar output signal from Test 1 where the subject is breathing normally while changing their orientation.

Figure 7.4 shows zoomed in plots (approximately 30 second windows) of the frontfacing, side-facing, and the back facing segments from Test 1. For the front-facing segment, there is a clear breathing signal with very good repeatability in the waveform shape. A breathing rate of roughly $0.22 \mathrm{~Hz}$ or 13 breaths per minute was calculated. For the side-facing segment, there appears to be a breathing signal, though it is not as clear as in the front-facing segment with poor repeatability in the waveform shape. A breathing rate of $0.27 \mathrm{~Hz}$ or 16 breaths per minute was calculated for the side-facing segment. For the back-facing segment, the breathing signal has poorer repeatability in the waveform shape than the front-facing segment, but better than the side-facing segment. A breathing rate of $0.2 \mathrm{~Hz}$ or 12 breaths per minute was calculated for the back-facing segment. Breathing rates were calculated using visual inspection. 

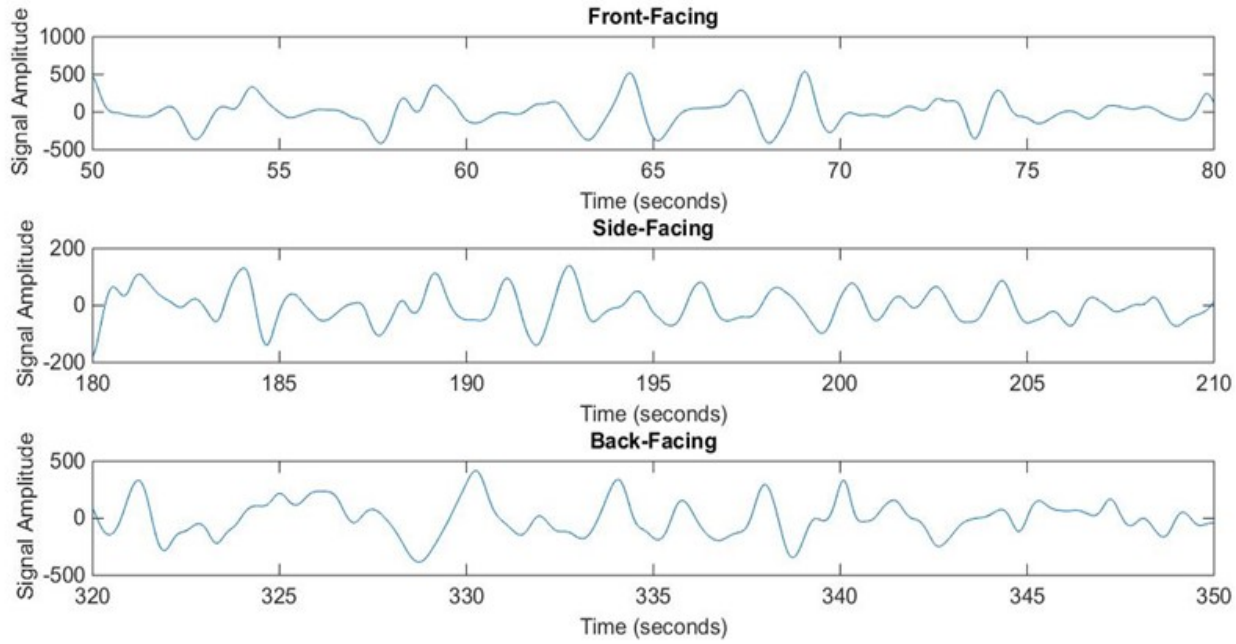

Figure 7.4 Zoomed in plots of the front-facing (top), side-facing (middle), and back-facing (bottom) segments from Test 1 .

Figure 7.5 shows the filtered radar output signal from the second test where the subject holds their breath during each segment. As in the first test, the three segments are labelled and can be clearly seen. The front-facing segment has a higher amplitude than the sidefacing segment and a similar amplitude compared to the back-facing segment.

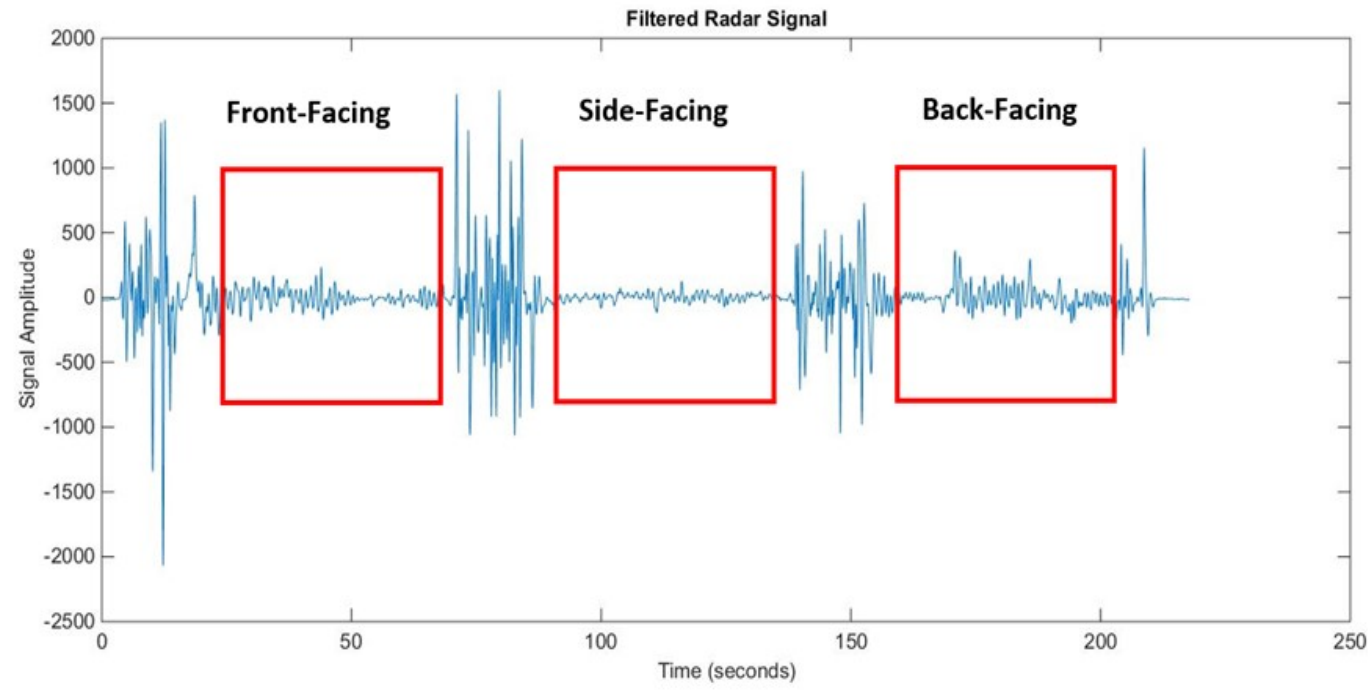

Figure 7.5 Filtered radar output signal from Test 2 where the subject is holding their breath after changing their orientation. 
Figure 7.6 shows zoomed in plots (approximately 30 second windows) of the frontfacing, side-facing, and the back facing segments from Test 2 .
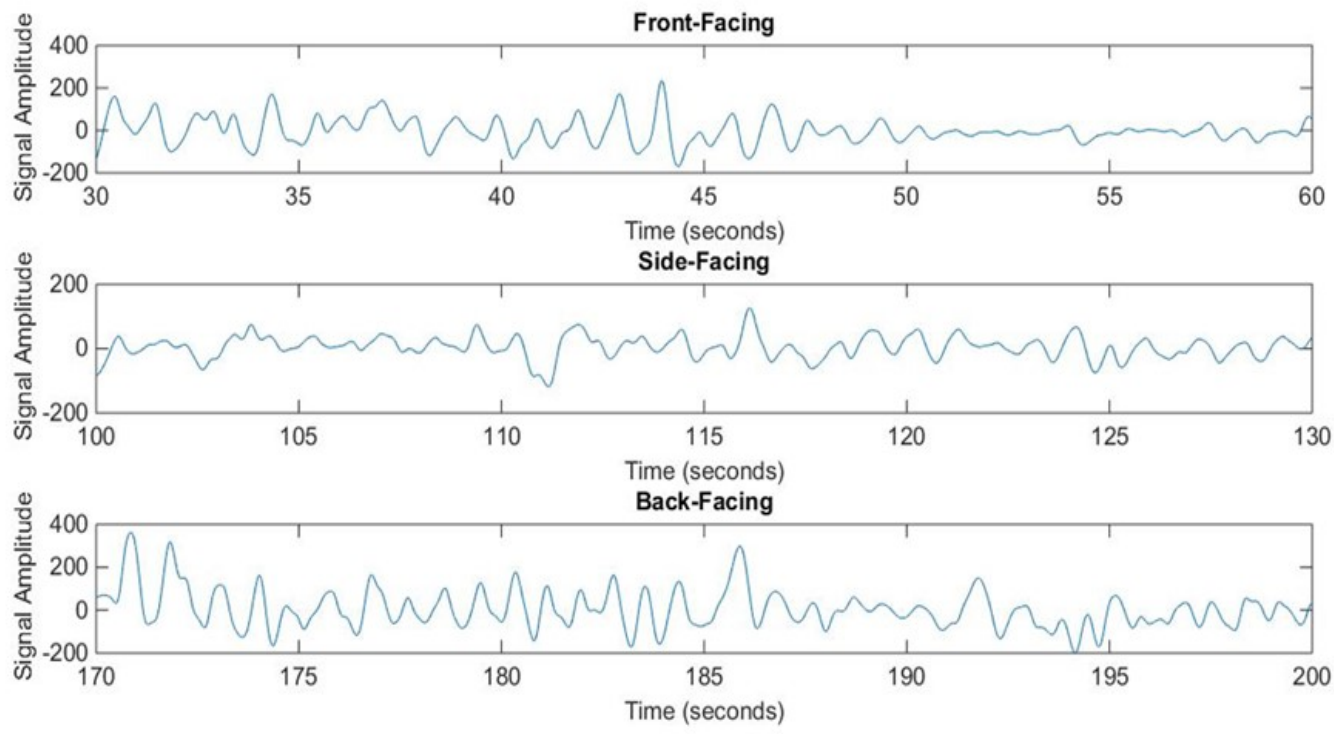

Figure 7.6 Zoomed in plots of the front-facing (top), side-facing (middle), and back-facing (bottom) segments from Test 2.

Figure 7.7 shows the magnitude spectrums for the front-, side-, and back-facing segments from Test 1 . The transition periods between each segment were not included in the spectral analysis. For the front-facing segment, there is a clear peak and another smaller peak around $0.22 \mathrm{~Hz}$ which likely corresponds to a breathing rate of 13 breaths per minute. This peak matches up with the breathing rate manually calculated for the frontfacing segment. For the side-facing segment, there is also a clear peak though it is outside the range of normal breathing. For the back-facing segment, there are two peaks with similar amplitudes though one is slightly greater than the other. Both peaks are within the normal breathing range. Table 7.2 lists the locations of the primary and secondary peaks from each of the segments. 

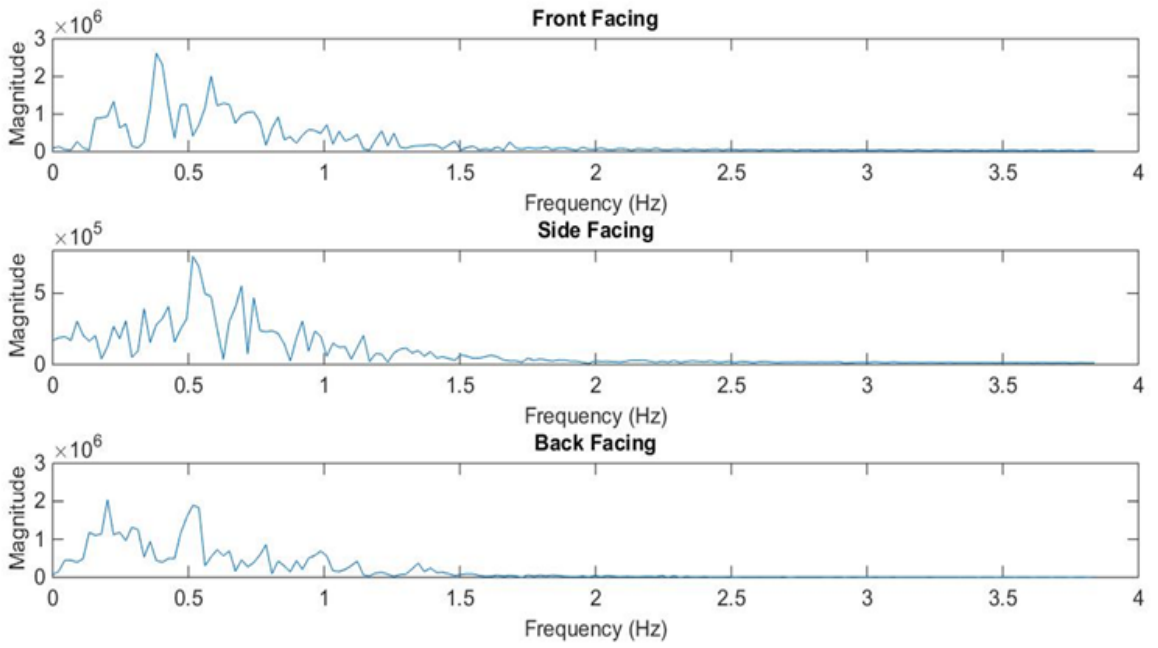

Figure 7.7 Magnitude spectrums for front-facing segment (top), side-facing segment (middle), and back-facing segment (bottom) from Test 1.

Table 7.2 Locations of primary and secondary peaks from Test 1.

\begin{tabular}{|c|c|c|c|c|}
\hline Segment & $\begin{array}{c}\text { Primary } \\
\text { Peak (Hz) }\end{array}$ & $\begin{array}{c}\text { Breathing Rate } \\
\text { (brpm) }\end{array}$ & $\begin{array}{c}\text { Secondary } \\
\text { Peak (Hz) }\end{array}$ & $\begin{array}{c}\text { Breathing } \\
\text { Rate (brpm) }\end{array}$ \\
\hline Front-facing & 0.38 & 22.8 & 0.22 & 13.2 \\
\hline Side-facing & 0.52 & 31.2 & 0.71 & 42.6 \\
\hline Back-facing & 0.20 & 12.0 & 0.51 & 30.6 \\
\hline
\end{tabular}

Figure 7.8 shows the magnitude spectrums of the breath hold sections for the front-, side, and back-facing segments from Test 2. Again, the transition periods between each segment were not included in the spectral analysis. Both the front and side-facing segments have clear, distinct peaks which are likely related to heart rates (as they fall within the expected range). For the back-facing segment, there are multiple peaks with similar amplitudes making it difficult to pick out a primary peak. Some of these peaks do fall within the expected range for heart rates. Table 7.3 lists the locations of the primary and secondary peaks from each of the segments. 

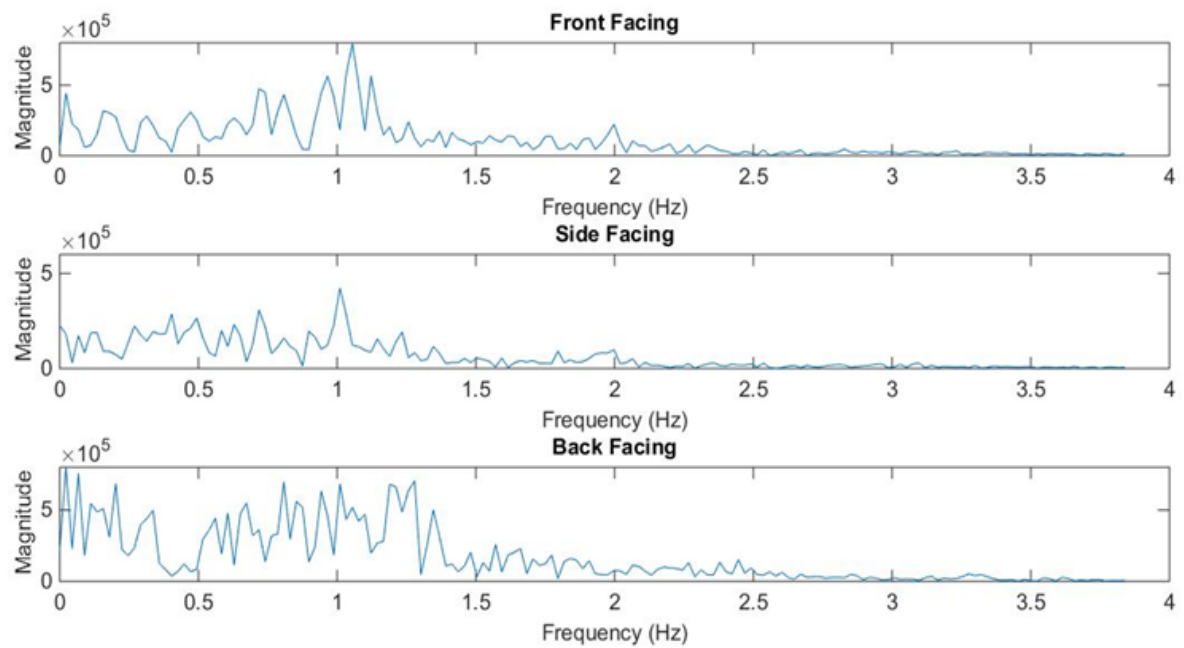

Figure 7.8 Magnitude spectrums for front-facing segment (top), side-facing segment (middle), and back-facing segment (bottom) from Test 2.

Table 7.3 Locations of primary and secondary peaks from Test 2.

\begin{tabular}{|c|c|c|c|c|}
\hline Segment & $\begin{array}{c}\text { Primary } \\
\text { peak (Hz) }\end{array}$ & $\begin{array}{c}\text { Heart Rate } \\
\mathbf{( b r p m )}\end{array}$ & $\begin{array}{c}\text { Secondary Peak } \\
\mathbf{( H z )}\end{array}$ & $\begin{array}{c}\text { Heart Rate } \\
\text { (brpm) }\end{array}$ \\
\hline Front-facing & 1.06 & 63.6 & 1.12 & 67.2 \\
\hline Side-facing & 1.01 & 60.6 & 0.72 & 43.2 \\
\hline Back-Facing & 0.02 & 1.2 & 1.01 & 60.6 \\
\hline
\end{tabular}

Figure 7.9 shows the filtered radar output signal from the third test where the subject moved the position of an RF panel in each of four one-minute segments. It is more difficult to distinguish the transition between the four segments, especially between segments three and four since they have similar signal amplitudes. During segment 2, when the RF panel is covering the abdomen, the signal amplitude decreases significantly. 


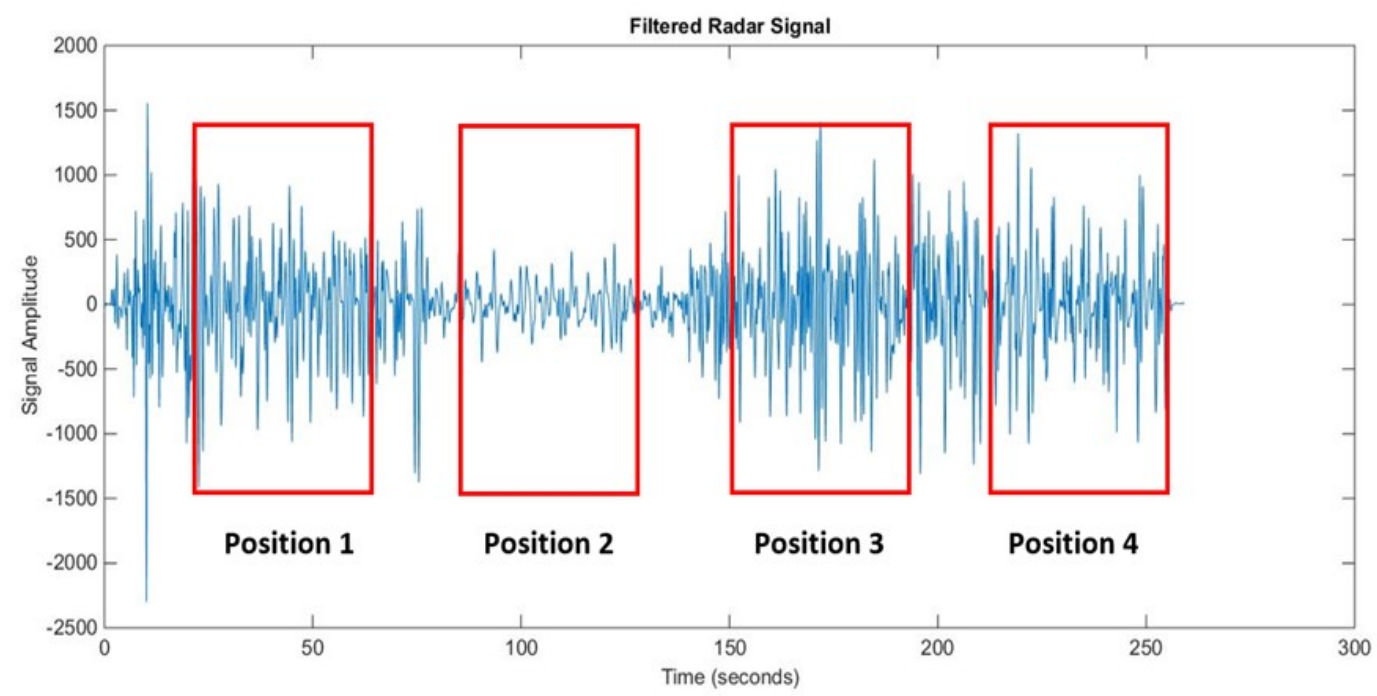

Figure 7.9 Filtered radar output signal from Test 3 where the subject is changing the position of an RF panel in each segment.

Figure 7.10 shows zoomed in plots (approximately 30 second windows) of the radar output signal from Test 3, where each plot is associated with one of the four positions of the RF panel. In positions 1, 3 and 4, the breathing signal is not very clear with very little repeatability in the waveform shape. For position 2, the breathing signal is clearer with better repeatability in the waveform shape. The estimated breathing rates (manually calculated using visual inspection) for positions 1-4 are 24, 14, 24, and 18 breaths per minute respectively, though it is difficult to estimate the breathing rates for positions 1,3 , and 4. 

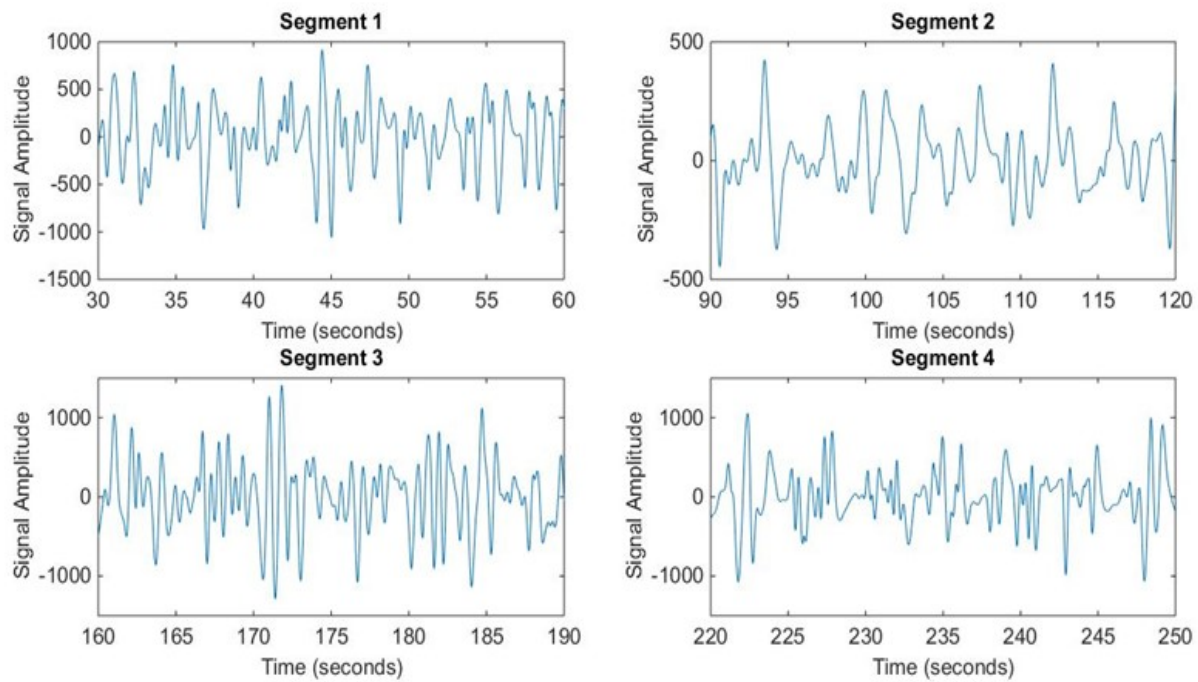

Figure 7.10 Zoomed in plots from Test 3 with the RF panel in position 1 (top left), position 2 (top right), position 3 (bottom left), and position 4 (bottom right).

Figure 7.11 shows the magnitude spectrums for each of the four segment from Test 3 associated with the position of the RF panel. The transition periods between each segment were not included in the spectral analysis. In position 1 there are three large peaks with similar amplitudes. In positions 2,3 , and 4 there are distinct primary peaks, along with one or two secondary peaks. In positions 2 and 4, the locations of the secondary peaks are likely related to breathing rate. Not only do these peak locations fall within the expected range, but they are also very similar to the rates manually calculated from the time domain signals. Table 7.4 lists the locations of the primary and secondary peak locations for all four segments. 

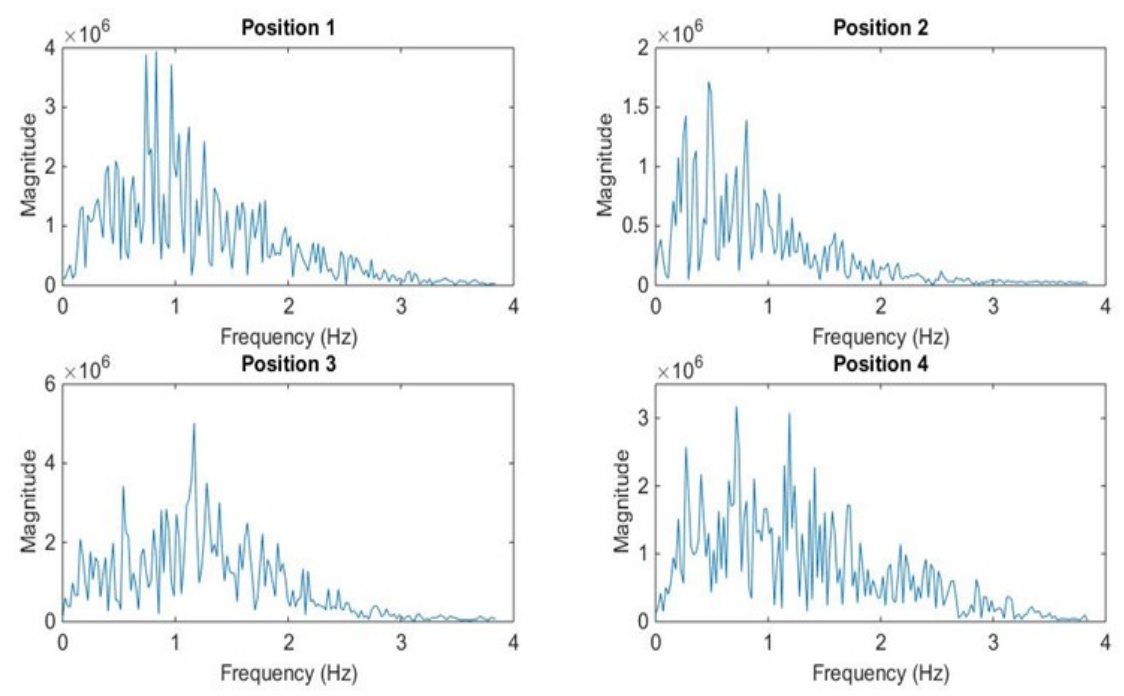

Figure 7.11 Magnitude spectrums from Test 3 with the RF panel in position 1 (top left), position 2 (top right), position 3 (bottom left), and position 4 (bottom right).

Table 7.4 Locations of primary and secondary peaks from Test 3.

\begin{tabular}{|c|c|c|c|c|}
\hline Segment & $\begin{array}{c}\text { Primary } \\
\text { Peak (Hz) }\end{array}$ & $\begin{array}{c}\text { Breathing/Heart } \\
\text { Rate (brpm/bpm) }\end{array}$ & $\begin{array}{c}\text { Secondary } \\
\text { Peak (Hz) }\end{array}$ & $\begin{array}{c}\text { Breathing/Heart } \\
\text { Rate (brpm/bpm) }\end{array}$ \\
\hline $\mathbf{1}$ & 0.83 & 49.8 & 0.74 & 44.4 \\
\hline $\mathbf{2}$ & 0.47 & 28.2 & 0.27 & 16.2 \\
\hline $\mathbf{3}$ & 1.17 & 70.2 & 0.54 & 32.4 \\
\hline $\mathbf{4}$ & 0.72 & 43.2 & 0.27 & 16.2 \\
\hline
\end{tabular}

\subsubsection{Analysis of Breathing Motion using Vicon Analysis}

Initially, it was clear that the subject was not completely still during the recordings, which meant that a straight calculation of the magnitude of movement of the markers would inaccurately represent the movement of the chest because it would include movement from the subject swaying. Movement related to swaying (translation, rotation, and shear) was removed using matrix transformations. The transformation in three reference markers (R1, R2, R5 for tests with subject lying down, and R1, R2, R6 for sitting and standing tests), which should be stationary during the recordings, was calculated from frame to frame, and the inverse transform was then applied to all 
markers, removing any swaying motion. The standard deviations of each marker (measured in millimeters) from each subject for each position were calculated. Figure 7.12 shows the average standard deviation values (in $\mathrm{mm}$ ) of each reference marker for each position. Although R4 was chosen as a reference marker because it was assumed that it would be stationary during breathing, this was not the case so it should not be used as a reference marker in future tests. The $\mathrm{R} 3$ and $\mathrm{R} 5$ markers move a little, but less than the other (non-reference) markers on the body.

\section{Reference Markers}

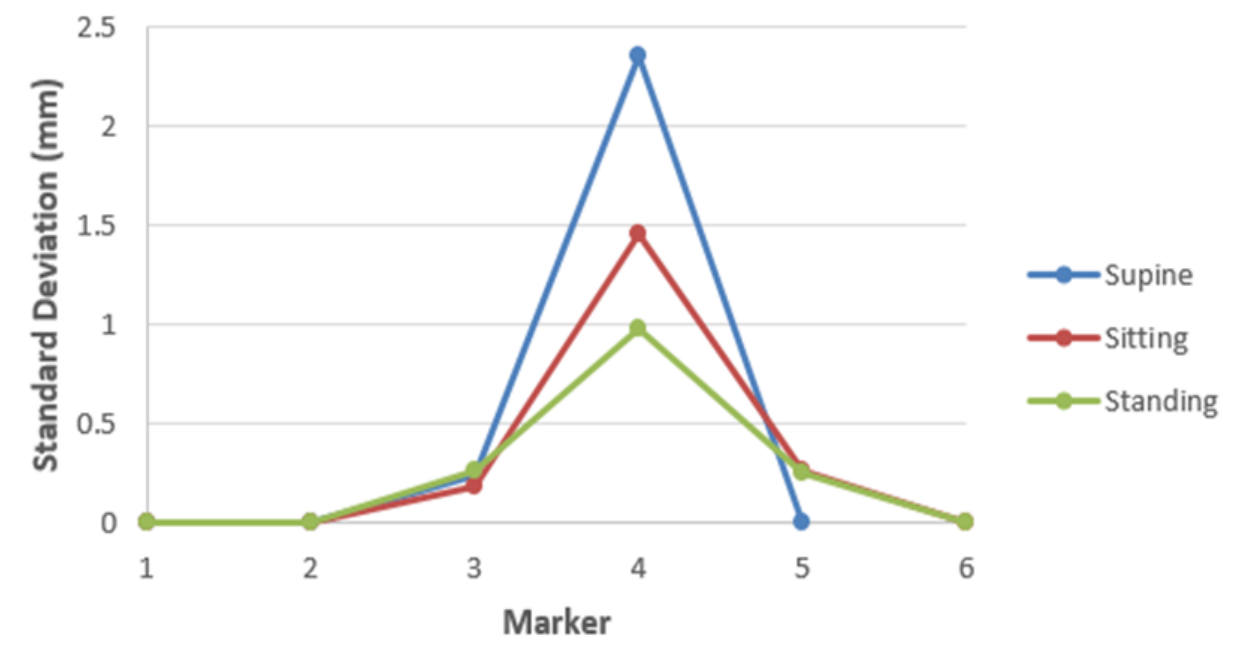

Figure 7.12 Standard deviations (in $\mathbf{m m}$ ) of the reference marker for each position.

Figures 7.13 shows the average standard deviation values (in $\mathrm{mm}$ ) of each marker from each subject during the recordings where the subjects were lying down (supine). The figure is color coded to better associate each set of points with their corresponding markers. 


\section{Supine Position}

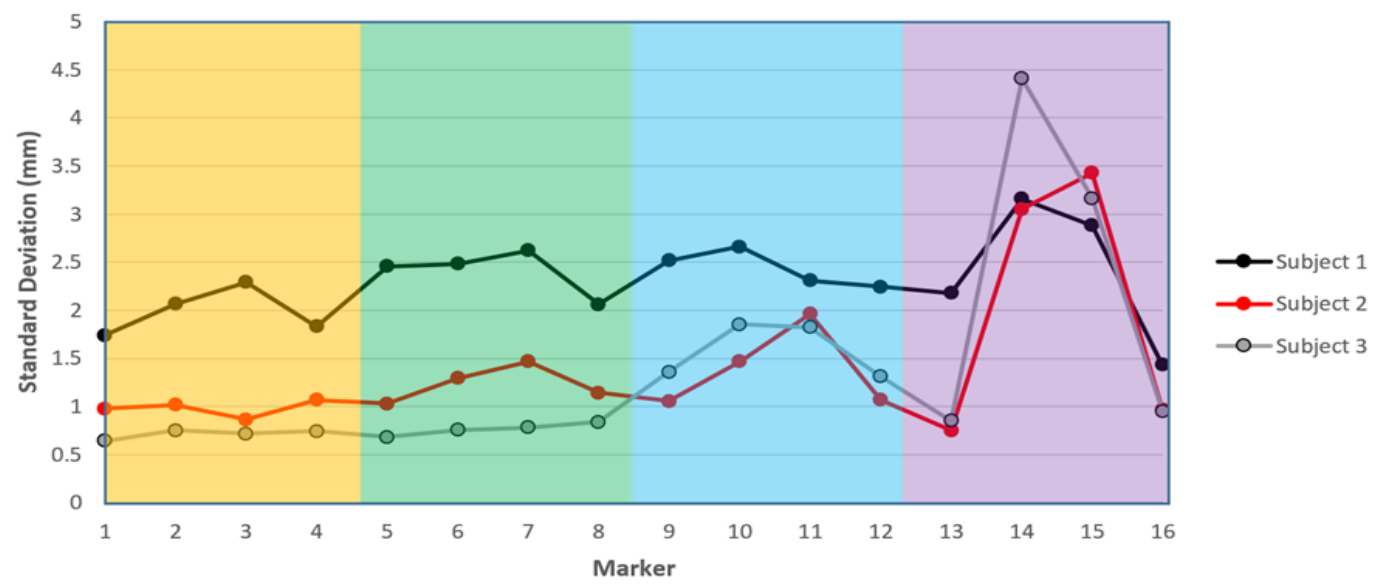

Figure 7.13 Standard deviations (in $\mathrm{mm}$ ) of each subject's markers when subjects were lying down.

Figures 7.14 shows the average standard deviation values (in $\mathrm{mm}$ ) of each marker from each subject during the recordings where the subjects were sitting. The figure is color coded to better associate each set of points with their corresponding markers. The figure shows that some markers for subject 2 are missing. The reason for this is that during the recordings, the cameras lost track of those markers throughout the recording. Because these markers had missing values throughout, the standard deviations were not calculated and left as unknown (blank).

\section{Sitting Position}

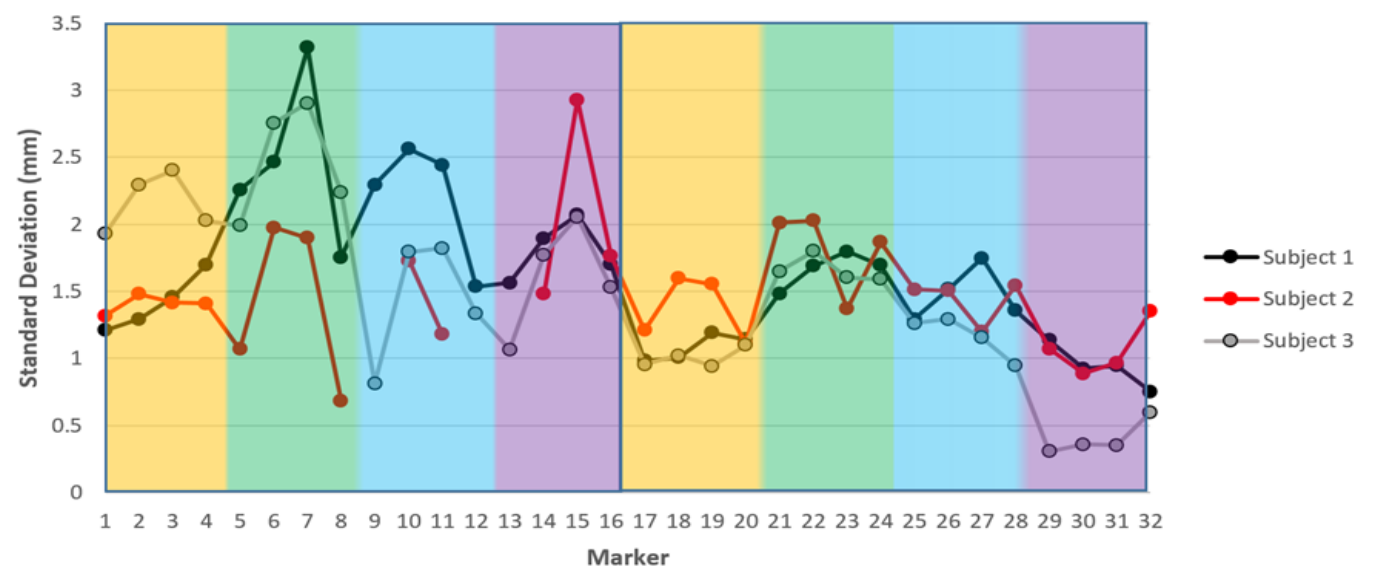

Figure 7.14 Standard deviations (in mm) of each subject's markers when subjects were seated. 
Figures 7.15 shows the average standard deviation values (in $\mathrm{mm}$ ) of each marker from each subject during the recordings where the subjects were standing. The figure is color coded to better associate each set of points with their corresponding markers. Many back markers from Subject 2 were lost by the cameras and did not provide adequate data.

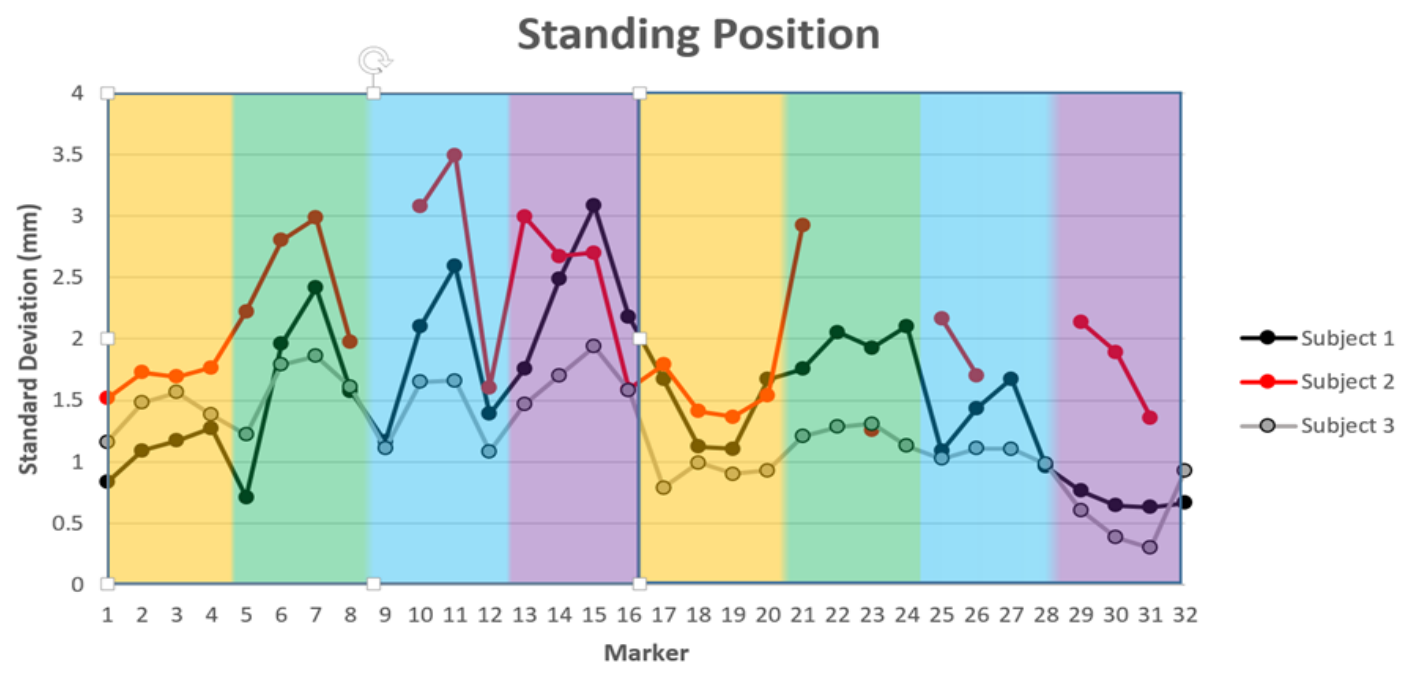

Figure 7.15 Standard deviations (in $\mathbf{m m}$ ) of each subject's markers when subjects were standing.

Figure 7.16 shows the overall mean standard deviation values of each marker in each position. These values were calculated by averaging the standard deviation values of each subject's markers from every test in each position.



Figure 7.16 Mean standard deviations (in $\mathrm{mm}$ ) of markers for each position. 


\subsection{Discussion}

\subsubsection{Effect of Orientation and Determination of Signal Source}

In the time domain, it can be seen that the best signal is obtained when the subject is facing the radar. This is not surprising since the radar can then most easily detect the motion of the upper body during breathing. The worst signal is obtained when the subject is facing sideways. When the subject has his back to the radar, the radar still appears to detect motion though it is not as strong as when the subject is facing the radar.

Looking at the magnitude spectrums in Figure 7.7 , the presence of similarly located primary peaks in the front-facing and back-facing segments indicates that the radar is capable of picking up slight breathing motion when pointed at the subject's back. In

Figure 7.8, the one clear peak at around $1 \mathrm{~Hz}$ in the front-facing segment spectrum, which corresponds to a heart rate of 60 beats per minute is evidence of the radar's ability to discern a person's heart rate. During the breath holds, the presence of identical primary peaks in the front and side-facing segments (refer to Table 7.3) could indicate that the radar can detect heartbeat from an area of the body other than the chest. In the backfacing segment, there are multiple peaks so the radar may not be able to detect a heartbeat when a person has their back to the radar unit. In all likelihood, the peaks are a result of small swaying motions which occur when a person sits without any back support. When a person is breathing regularly however, these small swaying motions may be masked by the motion resulting from breathing since there is a clear peak in Figure 7.7.

Time and frequency domain analysis of the radar output signal from the third test with the RF panels does not yield any clear results. The time domain signal is noisy in all positions of the RF panel, making it difficult to estimate the breathing rates through 
visual inspection. Additionally, the magnitude spectrums have primary peaks outside the range of normal breathing, and have multiple peaks with similar amplitudes which are also outside this range (as seen in Table 7.4). The noisy signal was likely a result of the experimental procedure. Throughout the test, the subject was asked to hold the RF panel; this could have introduced additional motion which the radar picked up, resulting in a noisier signal. If this test were to be repeated in the future, an apparatus for holding the RF panel in place should be constructed to reduce any noise or sources of error.

\subsubsection{Analysis of Breathing Motion using Vicon System}

Figures 7.13 and 7.16 show that when the subjects were in supine position, the markers located in the lower abdomen region moved the most while the markers located in the upper thorax moved the least. Not surprisingly, the markers located closer to the center of the body moved more than those closer to the sides of the body.

Figures 7.14 and 7.16 show that when subjects were sitting, the markers located in lower thorax region moved the most. Figure 7.14 does show variation in the movement patterns between subjects. This variation is likely caused by differences in body size and shape. There appears to be no consistent pattern in how the markers on the back move unlike the front markers where those closer to the center of the body move more than those closer to the sides.

Figures 7.15 and 7.16 show that when the subjects were standing, the markers on the lower thorax, lower and upper abdomen regions, move similar amounts. The magnitude that these markers move differs for every subject but again, this is likely due to differences in body shape and size. It is unclear why a few of the back markers moved more than the front markers. 
The Vicon data is comparable to results from existing literature. In [17], researchers found that markers on the abdomen moved more than markers on the lower and upper thorax when the subject was supine. Specifically, they found that the abdominal, lower thoracic, and upper thoracic markers had a mean standard deviation of $3.13 \mathrm{~mm}, 1.35$ $\mathrm{mm}$, and $1.45 \mathrm{~mm}$ respectively. The Vicon data (see Figure 7.16) shows similar values with the lower abdominal, lower thoracic, and upper thoracic markers having standard deviations of approximately $3.35 \mathrm{~mm}, 1.45 \mathrm{~mm}$, and $1.30 \mathrm{~mm}$ respectively. In both [20] and [21], researchers found similar patterns regarding how posture affects the magnitude of motion of abdominal and thoracic markers. When subjects were prone or sitting, the thoracic markers moved more than abdominal markers, but the opposite was true when the subjects were supine. Researchers measured diameter changes rather than standard deviations like in this thesis. The diameter change at the thorax was approximately $6 \mathrm{~mm}$ when the subject was sitting, and $3 \mathrm{~mm}$ when the subject was supine. The diameter change at the abdomen was approximately $7 \mathrm{~mm}$ when the subject was sitting, and $9 \mathrm{~mm}$ when the subject was supine. The Vicon data shows that the standard deviation of the thoracic markers is greater than for the abdominal markers when the subject is sitting, and that the abdominal markers have greater standard deviations than the thoracic markers when the subject is supine.

The Vicon data in Figure 7.16 also reinforces the observations from tests 1 and 2 (Section 7.3.1) that the strongest radar output signal will be obtained when the subject is facing the radar. The radar still detects motion when the subject has their back to the radar, although the signal will be weaker. The average standard deviation values for markers 1 to 16 (on the front of the subject's body) move more on average than markers 17 to 32 (on the 
subject's back). Because the markers on the front of the body move more than those on the back, this would result in a stronger radar output signal when the subject is facing the radar as opposed to when the subject's back is facing the radar. Though it is unlikely that a breathing signal would be noted from the back, the Vicon data does show motion in the upper thoracic area, which is consistent with the radar results.

The results also help to explain why the signal amplitude in Figure 7.9 from test 3 in section 7.3.1 (when the subject was seated) changes so significantly between segments two and three. In the second segment, the RF panel was covering the three lower regions and exposed some of the upper thorax, therefore, the signal amplitude was small. In the third segment, the lower abdomen and part of the space between the lower and upper abdomen were exposed resulting in a high amplitude signal. Finally, in the fourth segment, both the upper abdomen and lower thorax were uncovered resulting in a slightly higher amplitude signal.

\subsection{Conclusion}

This chapter investigated the source of the breathing signal and the motion of the upper body during regular breathing. The goals of the testing were to investigate how the subject's orientation affects the radar output signal, and which part of the body contributes the most to the radar output signal. These tests were carried out using the SR12003 radar unit as well as the Vicon motion tracking system.

The results show that the radar output signal is strongest when the subject is facing the radar, and weakest, when the subject has their side turned to the radar. The source of the radar's output signals is attributed to the movement of the upper body as evidence by the RF panel test. An objective analysis of breathing motion was conducted using the Vicon 
system and showed that sections of the upper body move different amounts, depending on whether the subject is lying down, sitting, or standing.

The preliminary results can help make future testing more efficient by specifying where the radar beam should target. Further experimentation should be done to investigate what the source of the radar output signal is. The test done with the RF panel should be repeated and improved to better isolate different sections of the upper body. Vicon testing was only done on three subjects so additional data would improve confidence and significance of the results. Coupling the data from the RF panel tests with the Vicon data would significantly improve our understanding of what the radar is picking up when displaying a breathing signal. 


\section{Chapter: Breathing Simulator}

\subsection{Introduction}

Data collection with human subjects is subject to high variability, both between subjects (inter-subject variability) and within subjects (intra-subject variability). Breathing movement is complex with various sections of the upper body moving in different directions and by different amounts. The source of the Doppler radar output signal is essentially unknown and virtually impossible to determine. Additionally, movements caused by the beating of the heart, as well as movement of the entire body (swaying) can affect the Doppler radar output signal.

As such, it would be ideal to perform data collection using a breathing simulator, which provides a known, repeatable motion pattern. This simulator would produce consistent results, and could be used to provide a better understanding of the radar's functionality. Preliminary work was done in developing a simulator using a linear actuator. This chapter describes testing done using the linear actuator. The preliminary testing investigated how the movement of the linear actuator affects the Doppler radar output signal, how the Doppler radar output signal compares to the velocity-time profile of the linear actuator, and how the range resolution of the radar affects the radar output signal.

\subsection{Breathing Simulator}

\subsubsection{Linear Actuator}

A linear actuator was used to provide a repeatable motion profile (the linear actuator can be programmed to cycle between two points; the motion in one cycle identical to the motion in any other cycle). The linear actuator that was employed was the Firgelli linear 
actuator (Model L16-P 100mm, Victoria, BC, Canada) shown in Figure 8.1. The specifications for the linear actuator (with a gear option of 150:1) are listed in Table 8.1.

Table 8.1 Specifications for the Firgelli linear actuator.

\begin{tabular}{|c|c|}
\hline Max Speed (no load) & $8 \mathrm{~mm} / \mathrm{s}$ \\
\hline Stroke Length & $100 \mathrm{~mm}$ \\
\hline Positional Accuracy & $0.4 \mathrm{~mm}$ \\
\hline Electrical Stroke & $98 \mathrm{~mm}$ \\
\hline Input Voltage & Rated at $12 \mathrm{VDC}$ \\
\hline
\end{tabular}

The L16-P is designed to push or pull a load along its full stroke length. The speed of travel is determined by the load applied (see the Load Curves in Figure 8.2 below). The different lines correspond to the different Gear options.

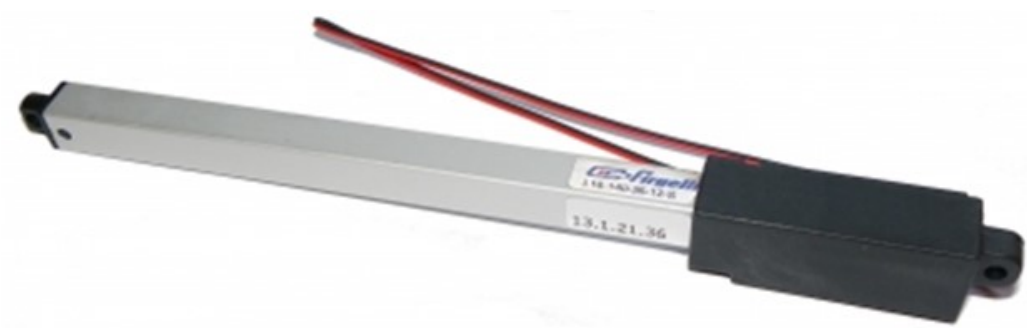

Figure 8.1 The Firgelli L16-P linear actuator.

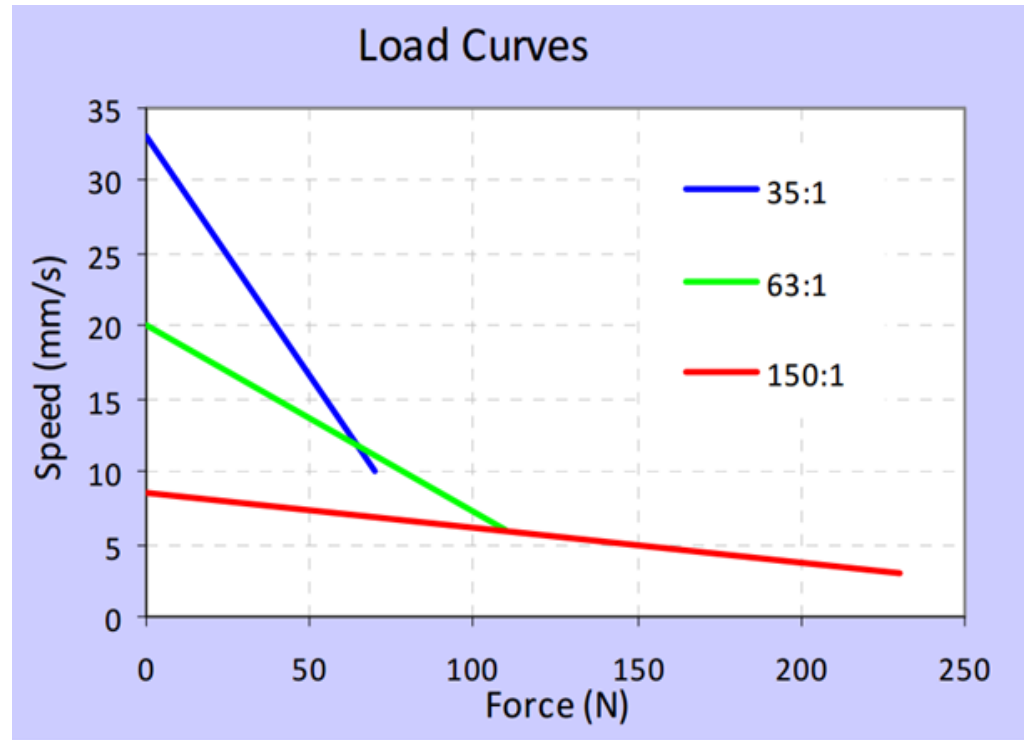

Figure 8.2 Load Curves for the L16-P miniature linear actuator. 


\subsubsection{Controller Board and Software}

The linear actuator is wired to a controller board (Model: Firgelli Linear Actuator Controller or LAC), which is connected to a computer/laptop via USB. The LAC Configuration Utility (see Figure 8.3) is used to control the position, speed, accuracy, extend, and retract limits of the linear actuator. Using the LAC Configuration Utility, one can change the position of the linear actuator or have the linear actuator automatically cycle between two positions. The position is set as a percentage of the linear actuator's stroke length, so a one percent change in position equals an extension or retraction of 1 $\mathrm{mm}$.

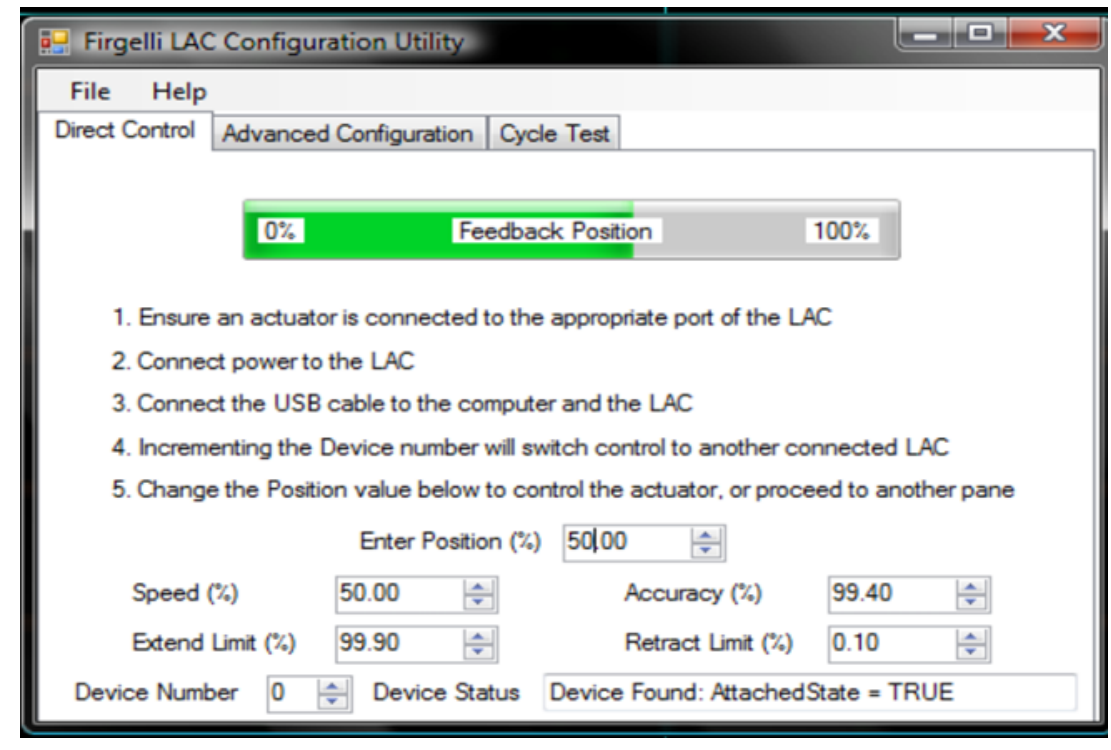

Figure 8.3 Firgelli LAC Configuration Utility program used to control the L16-P.

\subsubsection{Corner Reflector}

A corner reflector was attached to the linear actuator to increase the amount of radar waves reflected back to the radar unit (see Figure 8.4). 


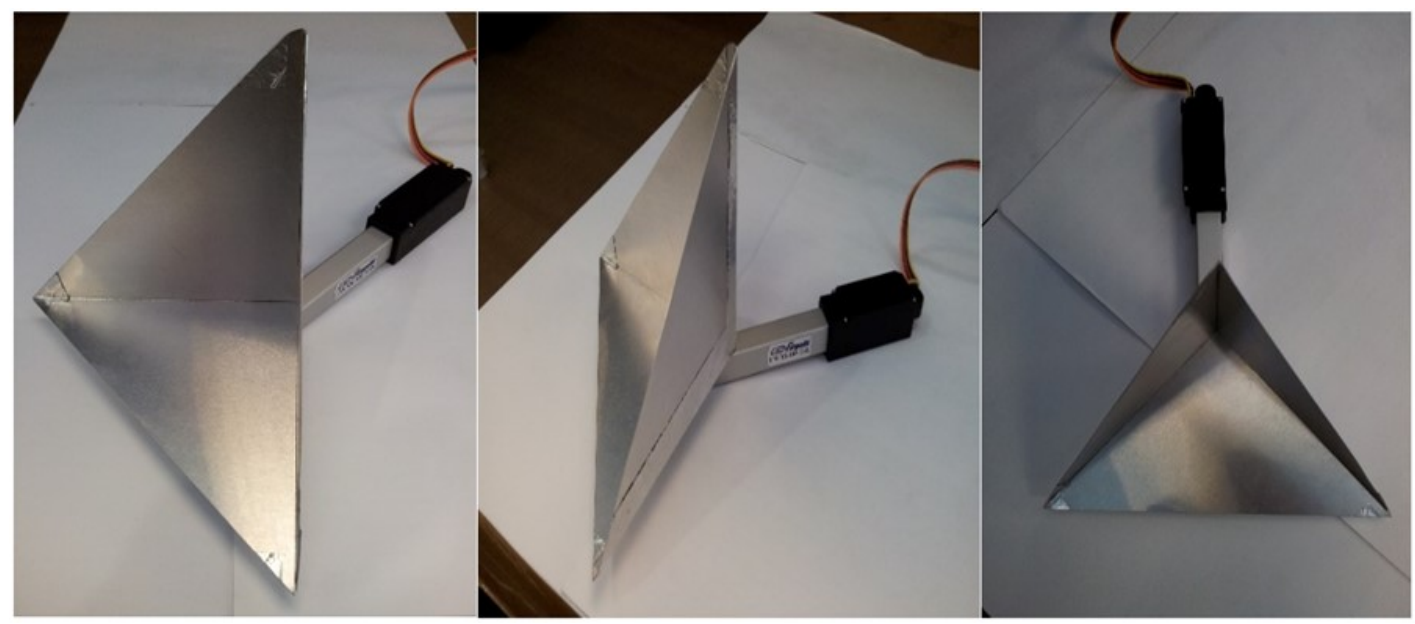

Figure 8.4 L16-P linear actuator with corner reflector attached.

\subsection{Methodology}

The linear actuator was placed in front of the radar unit such that it would extend towards and retract away from the radar. Four tests were conducted with the SR12003 radar unit oriented horizontally. The first two tests were conducted to investigate how the linear actuator movement affects the Doppler radar output signal, and how the signal compares to the linear actuator's time-velocity profile. The final two tests investigated how changing the range resolution of the radar unit affects the Doppler radar output signal. For all four tests, the speed of the linear actuator was set to $50 \%$ (approximately $4 \mathrm{~mm} / \mathrm{s}$ ), and the accuracy was set to $99.5 \%$. This was the highest accuracy that would prevent the linear actuator from oscillating above and below the set "Extend" and "Retract" limits.

\subsubsection{Velocity Information}

\subsubsection{Experimental Setup}

For tests 1 and 2, the linear actuator was positioned $50 \mathrm{~cm}$ (measured from the back corner of the reflector to the front of the radar unit) from the radar unit, optimizing the signal strength in Zone 3 (see Figure 8.5). The signal strength was optimized by shifting 
the radar unit a few centimetres towards and away from the linear actuator until the signal amplitude appeared to be at its maximum. Apart from setting the radar to Dwell on Zone 3 , the default range resolution and configuration parameters were used.

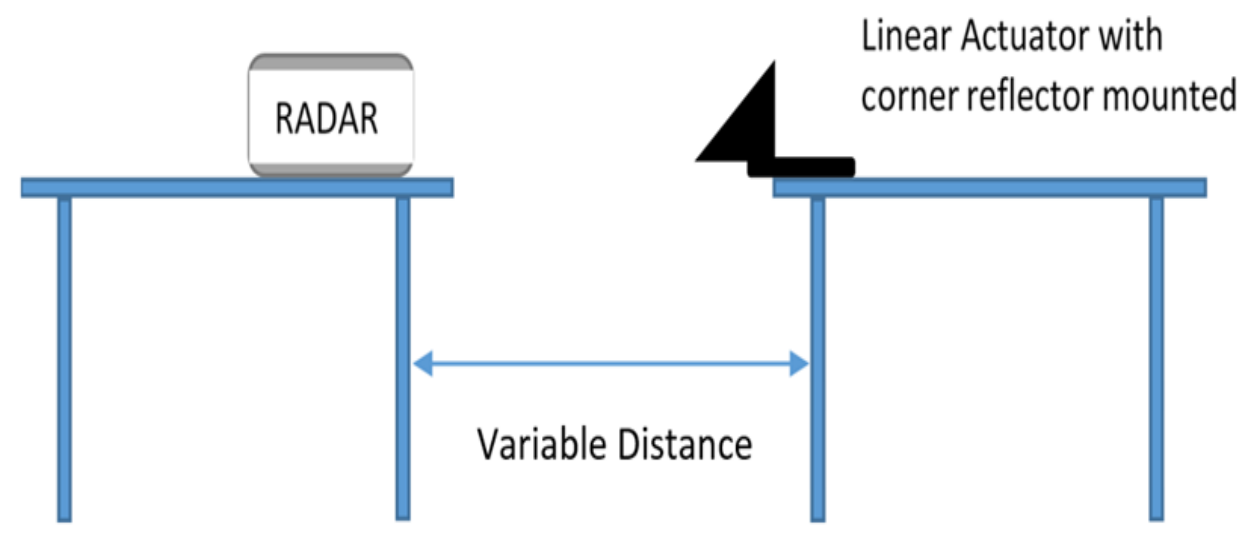

Figure 8.5 Experimental setup and placement of linear actuator relative to the radar unit for Tests 1 and 2.

\subsubsection{Experimental Procedure}

In test 1 , a manual cycle was performed. The linear actuator began at $64 \mathrm{~mm}$ (the extend limit). The position was then changed and the linear actuator retracted back to $50 \mathrm{~mm}$ (retract limit). After a short pause of approximately 7 seconds, the linear actuator was extended back to $64 \mathrm{~mm}$. This marked the end of the test.

In test 2, the linear actuator was set to run its automated cycle test, moving back and forth between the "Extend" limit $(50 \mathrm{~mm})$ and "Retract" limit $(64 \mathrm{~mm})$. The stop and start times of the radar and linear actuator were recorded for this test and can be seen below. The linear actuator was able to complete 1 cycle every 6.06 seconds or 0.165 cycles per second.

Radar start time: 12:08:15

Radar stop time: 12:14:05

Linear Actuator start time: 12:08:30

Linear Actuator stop time: 12:13:33 


\subsubsection{Effect of Range Resolution}

\subsubsection{Experimental Setup}

For tests 3 and 4, the radar was placed approximately 2.5 metres away from the radar when at its retract limit (see Figure 8.6). This distance was optimal for recording in Zone 5 when the range resolution was set to the default value of 0.75 metres (test 3 ), and Zone

1 when the range resolution was set to 3 metres (test 4).
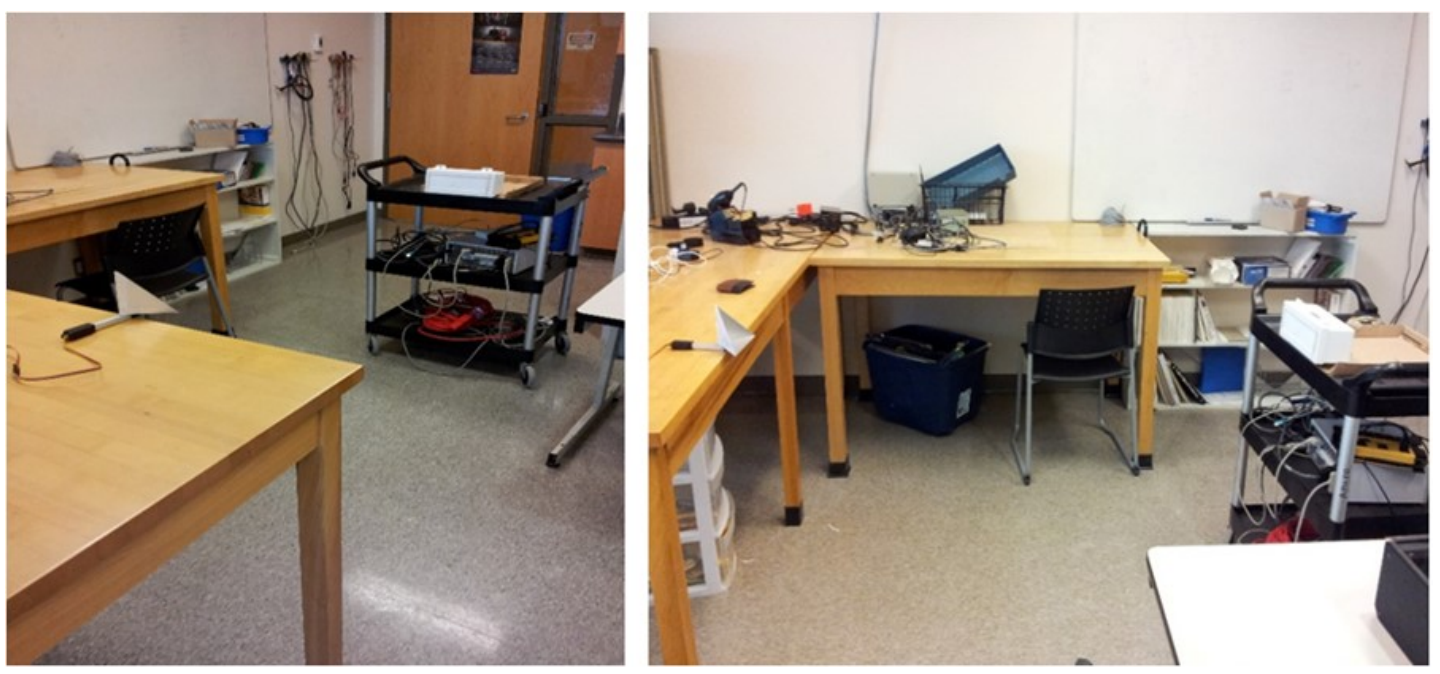

Figure 8.6 Experimental setup and placement of linear actuator relative to the radar unit for Tests 3 and 4 with views from behind the linear actuator (left) and from the side of the radar and linear actuator (right).

\subsubsection{Experimental Procedure}

For tests 3 and 4, the linear actuator was set to extend and retract $20 \mathrm{~mm}$ (from $50 \mathrm{~mm}$, the retract limit, to $70 \mathrm{~mm}$, the extend limit).

In test 3 , the radar was set to dwell on Zone 5 with a range resolution of 0.75 metres. The linear actuator was set to cycle and after approximately 30 seconds, was turned off. The radar was left running for a few more seconds to collect noise data. 
In test 4 , the radar was set to dwell on Zone 1 with a range resolution of 3 metres. Again, the linear actuator was set to cycle and after approximately 30 seconds, was turned off. The radar was left running for a few more seconds to collect noise data.

\subsection{Results}

\subsubsection{Velocity Information}

The radar output signals which recorded the linear actuator's movement were analysed in both the time and frequency domains. Figure 8.7 below shows the radar output signals from test 1 and 2 in the time domain. The top left plot depicts the manual cycle from test 1, while the bottom left plot shows the 50 automated cycles from test 2 . The top right plot shows three cycles from Test 2 with two cycles framed in red, and the bottom right plot shows the approximation of the linear actuator's velocity-time profile drawn over the same three cycles from the top right plot. The velocity-time profile was approximated using visual inspection and the knowledge that the linear actuator completes one cycle in approximately 6.06 seconds.
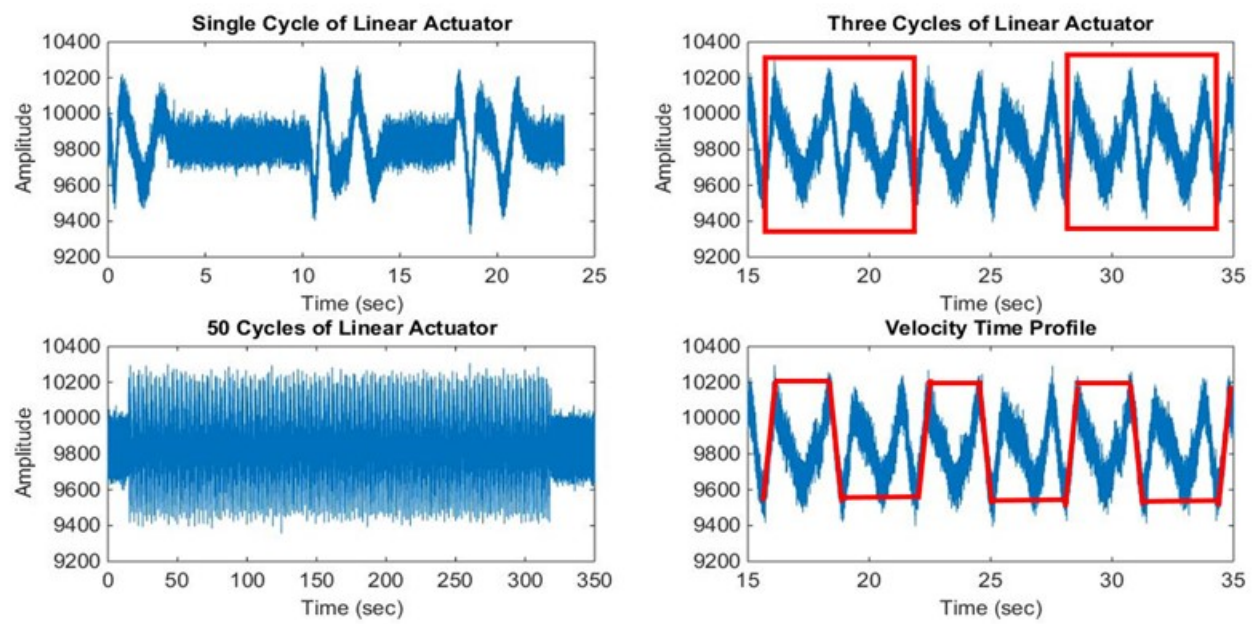

Figure 8.7 Radar output signals from the manual cycle from Test 1 (top left), the 50 automated cycles from Test 2 (bottom left), three cycles from the automated test with two cycles highlighted in red (top right), and the approximation of the velocity-time profile of the linear actuator (bottom right). 


\subsubsection{Effect of Range Resolution}

Figure 8.8 shows 20 second snapshots (from 65 seconds to 85 seconds) of the Zone 5 (top) and Zone 1 (middle) radar output signals from tests 3 and 4 respectively. The bottom plots both output signals over each other for easier comparison. The noise data collected at the end of the recordings appeared to be white noise.
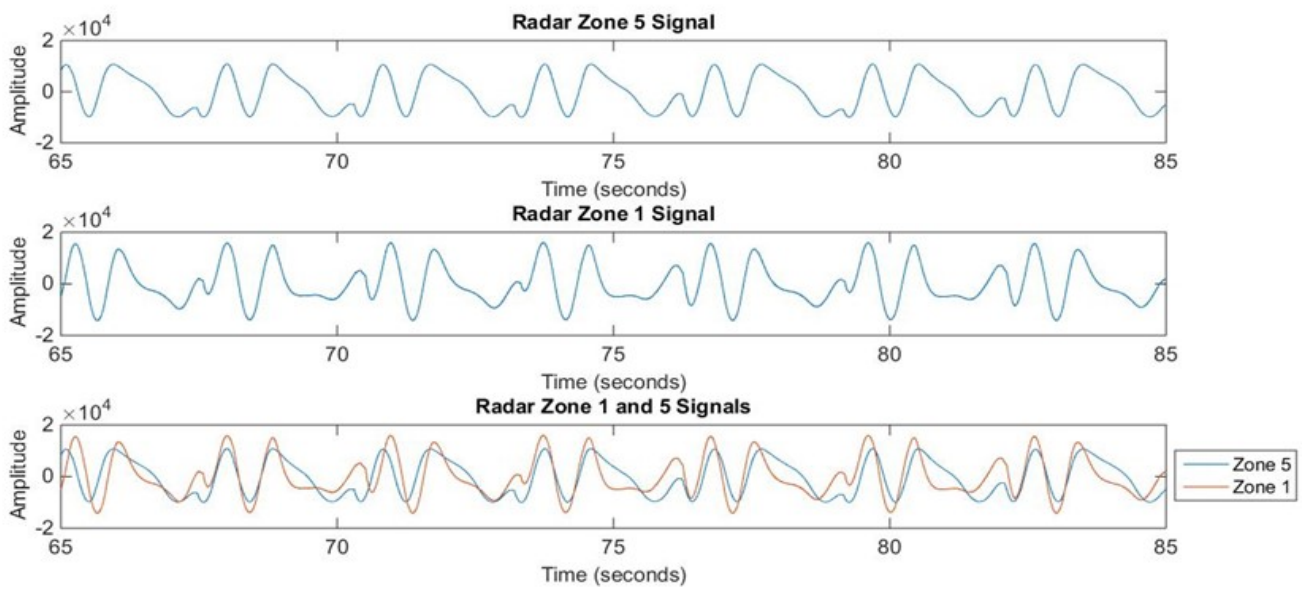

Figure 8.8 Twenty second snapshots of the Zone 5 (top), Zone 1 (middle) time domain signals from Tests 3 and 4 respectively, and the comparison between the two signal waveforms (bottom).

MATLAB was used to calculate the SNR values for the radar recordings from tests 3 and 4 by determining the $\mathrm{AC}$ power (variance) of the signal segments and noise segments, and then using equation 8.1 as follows:

$$
\begin{gathered}
\qquad S N R(d B)=10 \log \frac{P_{\text {signal }}}{P_{\text {noise }}} \text { (8.1) } \\
\text { where } P_{\text {signal }}=\text { signal AC power and } P_{\text {noise }}=\text { noise AC power }
\end{gathered}
$$

Table 8.2 below shows the AC power, and SNR values for both radar recordings.

Table 8.2 Signal and noise power values used to calculate the SNR of the radar output signals from Tests 3 and 4.

\begin{tabular}{|c|c|c|c|}
\hline Test & Signal Power & Noise Power & SNR (dB) \\
\hline $\mathbf{3}$ & $4.91 * 10^{7}$ & $4.59 * 10^{3}$ & 80.6 \\
\hline $\mathbf{4}$ & $6.00 * 10^{7}$ & $1.19 * 10^{4}$ & 74.0 \\
\hline
\end{tabular}




\subsection{Discussion}

\subsubsection{Velocity Information}

The top right plot from Figure 8.7 confirms that the linear actuator's movement produces repeatable patterns in the radar output signal. The two highlighted cycles have the same signal shape (velocity information) and length. The bottom right plot from Figure 8.7 plots the approximated velocity-time profile over the same three cycles of the automated test as in the top right plot. As the corner reflector moves away from the radar, its velocity increases until it reaches a constant velocity. As the corner reflector approaches the extend limit, it begins to decelerate (but since it is still moving away from the radar, it has a positive velocity). Once the corner reflector reaches the extend limit, it begins to move and accelerate back towards the radar (negative direction and therefore, negative velocity), before reaching a constant velocity. The corner reflector decelerates as it approaches the retract limit, and upon reaching it completes one cycle, and the cycle begins again. It is unclear why the radar output signal has additional oscillations where the velocity-time profile holds at a constant velocity (positive or negative).

\subsubsection{Effect of Range Resolution}

Comparing the two plots in figure 8.8, we see that the two signals have similar shapes. Small differences in signal shape as a result of the change in the range resolution (from 0.75 metres to 3 metres) are evident. With respect to the time domain, the smaller range resolution (0.75 metres) and consequently faster clock speed (200 MHz) produces oscillations with smaller amplitude than those produced with the larger range resolution (3 metres) and slower clock speed (50 MHz). Additionally, decreasing the radar's range resolution does bring small improvements to its Signal-to-Noise ratio. The ratio increases 
by a little over 6 decibels when the range resolution decreases from 3 metres ( $\mathrm{SNR}=74.0$

$\mathrm{dB})$ to 0.75 metres $(\mathrm{SNR}=80.6 \mathrm{~dB})$.

\subsection{Conclusion}

Testing with humans is subject to high variability which encouraged the progression of the research towards a motion source capable of repeating a specific movement. Testing was done using the linear actuator to develop a system that provides a repeatable motion pattern for testing. The results in this chapter represent preliminary work with this system. Tests were conducted to investigate if the Doppler radar output signal contains velocity information of the linear actuator's movement within its envelope. Additionally, the effect of changing the radar's range resolution was investigated.

The results appear to indicate that the velocity information of the linear actuator's movement can be determined from data found within the envelope of the Doppler radar output signal. Lowering the radar's range resolution to 0.75 metres produced more complete oscillations within the radar output signal, and improved the signal-to-noise ratio of the radar. Because the linear actuator's movement produces identical patterns in the radar output signal, it is reasonable to suggest that a person could write custom code to program any movement for the linear actuator. With a better understanding of breathing movement, one could potentially simulate breathing movement. Future work should explore this possibility. 


\section{Chapter: Conclusions and Recommendations for Future Work}

\subsection{Thesis Summary}

Correctional institutions are looking to adopt a non-obtrusive method for monitoring the vital signs (cardiorespiratory) of inmates in Canada. Doppler radar has been previously proposed as a potential method of vital signs detection and monitoring due to its applications in sleep studies and rescue operations. This thesis presents an algorithm implemented to estimate breathing rate from the Doppler radar output signal. The estimate is then compared with the estimate obtained from a respiratory inductance plethysmograph band to determine the accuracy of the estimate. Additionally, the estimate is validated with a reliability measure obtained through the analysis of different signal quality indices (signal-to-noise ratio, magnitude squared coherence, and variance). The radar output signal was also compared against collected electrocardiogram signals to investigate the radar's ability to detect heartbeat. Results indicated that the algorithm is capable of estimating breathing rate while a subject is stationary. During breath holds the radar appeared to pick up what looked to be heartbeat signals. Of the three signal quality indices, the signal-to-noise ratio appeared to be the best at quantifying signal quality, and performed the best as a reliability measure.

This thesis also presents an analysis of breathing movement of the upper body preformed using the Vicon motion capture system at Carleton University. This analysis was conducted to better understand what the radar is detecting/measuring during regular breathing. Subjects were recorded breathing in standing, sitting, and supine positions. Results show that the degree of abdominal and thoracic movement changes with respect to a person's position. When subjects were lying down, markers on the lower abdomen 
moved the most; when subjects were sitting, markers in the lower thoracic region moved the most. When subjects were standing, markers on the abdomen and lower thorax moved similar amounts. Anterior markers (on the front of the front of the subject's body), moved more on average than the posterior markers (on the subject's back). As such, the radar should work better when the subject is facing the radar. Preliminary work with a linear actuator was done to develop a repeatable motion system which could be used in the future to simulate breathing movement. Analysis of the radar output signal confirmed that the linear actuator could produce identical, oscillatory signals similar to a breathing signal.

\subsection{Future Work}

Despite obtaining encouraging results throughout the testing conducted within this thesis, further work is warranted in the following areas:

\section{Testing on a larger dataset}

Data should be collected from additional subjects for both the breathing estimation algorithm and the breathing motion analysis to validate the results and improve their statistical significance. In this thesis, data were collected from male subjects approximately the same age and with similar body types. Future testing should include a more diverse range of subjects, including female subjects, and subjects with varying body types, sizes, and ages.

\section{Applying more advanced signal processing techniques}

The breathing estimation algorithm presented in this thesis used basic signal processing techniques (frequency analysis, simple low- and high-pass filters) on the radar output signals, to establish a baseline of performance. Applying more advanced signal 
processing methods, such as harmonic analysis, and cross-correlation estimation versus FFT analysis, would certainly help improve the accuracy of the estimates as well as the reliability measure. In Chapter 5 it was shown that harmonic peaks are sometimes incorrectly associated with the breathing rate estimate, leading to large errors. With harmonic analysis, these errors can be reduced. Cross-correlation could help because it looks at the repeating pulse shape associated with each breath, which could reduce errors associated with harmonics.

\section{Real-time Implementation}

The algorithm presented in this thesis estimates the breathing rate post data collection. Future work should attempt real-time implementation of the algorithm. Additionally, an alarm system should be developed which generates an alarm when the estimates are outside the normal bounds for breathing rate and the reliability of the estimate is high.

\section{Multi Radar System}

Development of a multi radar system should be explored. The use of two radars could allow for triangulation of a subject's position. In this thesis, the use of a single radar only provided ranging capabilities as opposed to localization capabilities. This thesis also found that a person's orientation to the radar (front, side, or backwards facing) does have an effect on the quality of the radar output signal and the radar's ability to detect breathing motion. With one radar, orientation plays a large factor in life signs detection, but with two or more radars, orientation plays a smaller factor because it is more likely that the subject under observation will be facing at least one radar at any time. Additionally, with a multi radar system, each radar could provide a breathing rate estimate and the SQIs from each radar could be used to combine the estimates from both 
radars. If one radar has a high reliability in the estimate and the other has a low reliability, the breathing estimate from the radar with the lower reliability can be discarded.

\section{Repeatable motion analysis}

Further testing should be done with the linear actuator. Breathing motion analysis of data from human subjects had to contend with inter- and intra-subject variability. Using the breathing movement analysis data presented in this thesis, a breathing simulator could be developed by programming the linear actuator to move in the same manner as a person's abdomen or thorax. The linear actuator would provide an extensive amount of data for analysis because of its ability to repeat the same physical motion, removing any variability stemming from the subject/target under observation. In addition, as the motion of the linear actuator is well-controlled and defined, it would be easier to relate the radar output signal to the detected motion. In human subjects, breathing motion is complex (e.g., multiple moving body parts) and thus difficult to relate to the radar output signal. 


\section{References}

[1] K. Harris, "Suicide attempts, self-harm rising in Canada's prisons," CBC News, 23 October 2012. [Online]. Available: http://www.cbc.ca/news/politics/suicideattempts-self-harm-rising-in-canada-s-prisons-1.1163978. [Accessed 10 June 2014].

[2] K. Harris, "Self-harm incidents among female prisoners highest in Prairies," CBC News, 18 March 2015. [Online]. Available: http://www.cbc.ca/news/politics/selfharm-incidents-among-female-prisoners-highest-in-prairies-1.3000397. [Accessed 13 October 2015].

[3] "Prisoner Self-Injury On The Rise In Canada," Huffington Post, 11 October 2012. [Online]. Available: http://www.huffingtonpost.ca/2012/10/11/prisoner-self-injuryin-canada-_n_1959895.html.

[4] H. Sapers, "Annual Report of the Office of the Correctional Investigator 20112012," Ottawa, 2012.

[5] A. E. Daniel, "Preventing suicide in prison: a collaborative responsibility of administrative, custodial, and clinical staff," Journal of the American Academy of Psychiatry and the Law Online, vol. 34, no. 2, pp. 165-175, 2006.

[6] D. Gunnell, "The epidemiology and prevention of suicide by hanging: a systematic review," International Journal of Epidemiology, vol. 34, no. 2, pp. 433-442, 2005.

[7] C. Graichen, J. Ashe, M. Ganesh and L. Yu, "Unobtrusive vital signs monitoring with range-controlled radar," in IEEE Signal Processing in Medicine and Biology Symposium (SPMB), New York, 2012.

[8] S. I. Fox, Fundamentals of Human Physiology, New York: McGraw-Hill, 2009.

[9] P. Lavelle, "Lung Cancer," ABC Health and Well Being, 29 July 2010. [Online]. Available: http://www.abc.net.au/health/library/stories/2010/07/29/2967396.htm. [Accessed 25 November 2015].

[10] J. G. Webster, Medical Instrumentation Applications and Design, Hoboken: John Wiley and Sons, Inc., 2010.

[11] G. Ferrigno, P. Carnevali, A. Aliverti, F. Molteni, G. Beulcke and A. Pedotti, "Three-Dimensional Optical Analysis of Chest Wall Motion," Journal of Applied Physiology, vol. 77, no. 3, pp. 1224-1231, 1994.

[12] S. J. Cala, C. M. Kenyon, G. Ferrigno, P. Carnevali and A. Aliverti, "Chest Wall and Lung Volume Estimation by Optical Reflectance Motion Analysis," Journal of Applied Physiology, vol. 81, no. 6, pp. 2680-2689, 1996. 
[13] K. S. Tan, R. Saatchi, H. Elphick and D. Burke, "Real-Time Vision Based Respiration Monitoring System," in 7th International Symposium on Communication System Networks and Digital Signal Processing (CSNDSP), Poznan, 2010.

[14] M. F. Olsen and K. Romberg, "Reliability of the Respiratory Movement Measuring Instrument, RMMI: Reliability of the RMMI," Clinical Physiology and Functional Imaging, vol. 30, no. 5, pp. 349-353, 2010.

[15] H. Kaneko, "Estimating Breathing Movements of the Chest and Abdominal Wall Using a Simple, Newly Developed Breathing Movement Measuring Device," Respiratory Care, vol. 59, no. 7, pp. 1133-1139, 2014.

[16] H. Kaneko, "Assessing the Reliability and Validity of a Newly Developed Breathing Movement Measuring Device," Journal of Physical Therapy Science, vol. 25 , no. 4, pp. 425-429, 2013.

[17] M. Ragnarsdottir and E. K. Kristinsdottir, "Breathing Movements and Breathing Patterns among Healthy Men and Women 20-69 Years of Age," Respiration, vol. 73, no. 1, pp. 48-54, 2006.

[18] J. T. Sharp, N. B. Goldberg, W. S. Druz and J. Danon, "Relative Contributions of Rib Cage and Abdomen to Breathing in Normal Subjects," Journal of Applied Physiology, vol. 39, no. 4, pp. 608-618, 1975.

[19] G. Shafiq and K. C. Veluvolu, "Surface Chest Motion Decomposition for Cardiovascular Monitoring," Scientific Reports, vol. 4, pp. 1-9, 2014.

[20] A. Aliverti, R. Dellaca, P. Pelosi and D. Chiumello, "Compartmental Analysis of Breathing in the Supine and Prone Positions by Optoelectronic Plethysmography," Annals of Biomedical Engineering, vol. 29, no. 1, pp. 60-70, 2001.

[21] M. Romei, A. Mauro, M. G. D'Angelo, A. C. Turconi, N. Bresolin, A. Pedotti and A. Aliverti, "Effects of Gender and Posture on Thoraco-Abdominal Kinematics During Quiet Breathing in Healthy Adults," Respiratory Physiology \& Neurobiology, vol. 172, no. 3, pp. 184-191, 2010.

[22] R. Plonsey and J. Malmivuo, Bioelectromagnetism - Principles and Applications of Bioelectric and Biomagnetic Fields, New York: Oxford University Press, 1995.

[23] J. Toomay and P. J. Hannen, Radar Principles for the Non-specialist, Third ed., Raleigh: SciTech Publishing Inc., 2004.

[24] C. Wolff, "Waves and Frequency Ranges," Radar Basics - radartutorial.eu, [Online]. Available: http://www.radartutorial.eu/07.waves/Waves\%20and\%20Frequency\%20Ranges.en .html. [Accessed 10 November 2015].

[25] A. M. Ponsford, "Introduction to Radar," Raytheon Company, Ottawa, 2014. 
[26] V. Chen, The Micro-Doppler Effect in Radar, Norwood: Artec House, 2011.

[27] B. R. Mahafza, Radar Systems Analysis and Design Using Matlab, Boca Raton: Chapman \& Hall/CRC, 2000.

[28] D. Dei, G. Grazzini, G. Luzi, M. Pieraccini and C. Atzeni, "Non-Contact Detection of Breathing Using a Microwave Sensor," Sensors, vol. 9, no. 1, pp. 2574-2585, 2009.

[29] J. D. Taylor, Introduction to Ultra-Wideband Radar Systems, Boca Raton: CRC Press, 1994.

[30] C. Gabriel, S. Gabriel and E. Corthout, "The dielectric properties of biological tissues: I. Literature survey," Physics in Medicine and Biology, vol. 41, no. 11, pp. 2231-2249, 1996.

[31] N. M. Tamyis, D. K. Ghodgaonkar, N. Taib and W. Tin Wui, "Dielectric Properties of Human Skin In Vivo in the Frequency Range 20-38 GHz for 42 Healthy Volunteers," Proceedings of the 28th URSI General Assembly, 2005.

[32] K. Sasaki, K. Wake, S. Watanabe and M. Mizuno, "Dielectric Property measurements of biological tissues: Recent activities for development of a novel database," in IEEE General Assembly and Scientific Symposium XXXI URSI, Beijing, 2014.

[33] E. M. Staderini, "UWB Radars in Medicine," IEEE Aerospace and Electronic Systems Magazine, vol. 17, no. 1, pp. 13-18, 2002.

[34] C. Li and J. Lin, "Recent Advances in Doppler Radar Sensors for Pervasive Healthcare Monitoring," in IEEE Proceedings of Asia-Pacific Microwave Conference, Yokohama, 2010.

[35] C. Li, V. M. Lubecke, O. Boric-Lubecke and J. Lin, "A Review on Recent Advances in Doppler Radar Sensors for Noncontact Health Monitoring," IEEE Transactions on Microwave Theory and Techniques, vol. 61, no. 5, pp. 2046-2060, 2013.

[36] L. Crocco and V. Ferrara, "A review on ground penetrating radar technology for the detection of buried or trapped victims," in International Conference on Collaboration Technologies and Systems (CTS), Minneapolis, 2014.

[37] G. Vinci, S. Lindner, F. Barbon, H. M., G. Fischer, D. Kissinger and A. Koelpin, "24 GHz Six-Port Medical Radar for Contactless Respiration Detection and Heartbeat Monitoring," in Proceedings of the 9th European Radar Conference, Amsterdam, 2012.

[38] G. Sun, M. Kubota, M. Kagawa, N. Q. Vinh, A. Kurita and T. Matsui, "A Screening Method Based on Amplitude Probability Distribution Analysis for Detecting the Disordered Breathing Using Microwave Radar Respiration Signals," 
in Proceedings from the Asia-Pacific Microwave Conference (APMC), Kaohsiung, 2012.

[39] B. J. Jang, S. H. Wi, J. G. Yook, M. Q. Lee and K. J. Lee, "Wirless Bio-Radar Sensor for Heartbeat and Respiration Detection," Progress in Electromagnetic Research C, vol. 5, pp. 149-168, 2008.

[40] V. Das, A. Boothby, R. Hwang, T. Nguyen, J. Lopez and D. Y. C. Lie, "Antenna Evaluation of a Non-Contact Vital Signs Sensor for Continous Heart and Respiration Rate Monitoring," in IEEE Topical Conference on Biomedical Wireless Technologies, Networks, and Sensing Systems (BioWireleSS), Santa Clara, 2012.

[41] O. Postolache, P. S. Girao, R. N. Madeira and G. Postolache, "Microwave FMCW Doppler Radar Implementation for In-House Pervasive Health Care System," in IEEE International Workshop on Medical Measurements and Applications Proceedings (MeMeA), Ottawa, 2010.

[42] I. Y. Immoreev, P. S. V. Samkov and T.-H. Tao, "Short-Distance Ultra-Wideband Radars Theory and Designing," in International Conference on Radar Systems, Philadelphia, 2004.

[43] Y. Wang, Q. Liu and A. E. Fathy, "Simultaneous Localization and Respiration Detection of Multiple People Using Low Cost UWB Biometric Pulse Doppler Radar Sensor," in IEEE International Microwave Symposium (MTT), Montreal, 2012.

[44] C. Gu, R. Li, S. B. Jiang and C. Li, "A Multi-Radar Wireless System for Respiratory Gating and Accurate Tumor Tracking in Lung Cancer Radiotherapy," in 33rd Annual International Conference of the IEEE EMBS, Boston, 2011.

[45] R. F. Fouladi and A. Oncu, "Vital Signs Modeling for Doppler Radar Cardiorespiratory Monitoring," in 36th International Conference on Telecommunications and Signal Processing (TSP), Rome, 2013.

[46] C. Li and J. Lin, "Random Body Movement Cancellation in Doppler Radar Vital Sign Detection," IEEE Transactions on Microwave Theory and Techniques, vol. 56, no. 12, pp. 3143-3152, 2008.

[47] A. Rahman, E. Yavari, X. Gao, V. Lubecke and O. Boric-Lubecke, "Signal Processing Techniques for Vital Sign Monitoring Using Mobile Short Range Doppler Radar," in IEEE Topical Conference on Biomedical Wireless Technologies, Networks, and Sensing Systems (BioWireleSS), San Diego, 2015.

[48] L. Chioukh, H. Boutayeb, D. Deslandes and K. Wu, "Noise and Sensitivity of Harmonic Radar Architecture for Remote Sensing and Detection of Vital Signs," IEEE Transactions on Microwave Theory and Techniques, vol. 62, no. 9, pp. 18471855, 2014. 
[49] A. Tariq and H. G. Shiraz, "Doppler Radar Vital Signs Monitoring using Wavelet Transform," in 2010 Loughborough Antennas \& Propagation Conference, Loughborough, 2010.

[50] J.-H. Lee, J. M. Hwang, D. H. Choi and S.-O. Park, "Noninvasive Biosignal Detection Radar System Using Circular Polarization," IEEE Transactions on Information Technology in Biomedicine, vol. 13, no. 3, pp. 400-404, 2009.

[51] C. Song, E. Yavari, A. Singh, O. Boric-Lubecke and V. Lubecke, "Detection Sensitivity and Power Consumption vs. Operation Modes Using System-On-Chip Based Doppler Radar Occupancy Sensor," in IEEE Topical Conference on Biomedical Wireless Technologies, Networks, and Sensing Systems (BioWireleSS), Santa Clara, 2012.

[52] C. Joslin, "Motion Capture Vicon MX/T-Series System," Carleton University, 2008. [Online]. Available: http://mocap.csit.carleton.ca/. [Accessed 1 February 2015].

[53] A. Droitcour, O. Boric-Lubecke, V. Lubecke, J. Lin and G. Kovacs, "Range Correlation and I/Q Performance Benefits in Single-Chip Silicon Doppler Radars for Noncontact Cardiopulmonary Monitoring," IEEE Transactions on Microwave Theory and Techniques, vol. 52, no. 3, pp. 838-848, 2004.

[54] B.-K. Park, O. Boric-Lubecke and V. Lubecke, "Arctangent Demodulation with DC Offset Compensation in Quadrature Doppler Receiver Systems," IEEE Transactions on Microwave Theory and Techniques, vol. 55, no. 5, pp. 1073-1079, 2007.

[55] A. D. Droitcour, O. Boris-Lubecke, V. M. Lubecke, J. Lin and G. T. A. Kovacs, "Range Correlation Effect on ISM Band and I/Q CMOS Radar for Non-Contact Vital Sign Sensing," in IEEE MTT-S International Microwave Symposium Digest, Philadelphia, 2003.

[56] "Vital Signs," Cleveland Clinic, 25 April 2014. [Online]. Available: https://my.clevelandclinic.org/health/healthy_living/hic_Preparticipation_Evaluations/hic_Vital_Signs. [Accessed 30 November 2015].

[57] "Calm Breathing," AnxietyBC, [Online]. Available: http://www.anxietybc.com/sites/default/files/CalmBreathing.pdf.

[58] "mscohere," MathWorks, [Online]. Available: http://www.mathworks.com/help/signal/ref/mscohere.html. [Accessed 15 November 2015].

"var," MathWorks, [Online]. Available: http://www.mathworks.com/help/matlab/ref/var.html. [Accessed 15 November 2015]. 
[60] "Human Body Outline Front and Back Drawing," ClipArt Best, [Online]. Available: http://www.clipartbest.com/clipart-pi5eddriB. [Accessed 10 October 2015]. 